\title{
Low Energy Supergravity Revisited (I)
}

\author{
Gilbert Moultaka, ${ }^{1, *}$ Michel Rausch de Traubenberg, ${ }^{2, \dagger}$ and Damien Tant ${ }^{2, \ddagger}$ \\ ${ }^{1}$ Laboratoire Charles Coulomb (L2C), UMR 5221 CNRS-Université de Montpellier, Montpellier, F-France \\ ${ }^{2}$ Université de Strasbourg, CNRS, IPHC UMR 7178, F-6700 Strasbourg, France
}

(Dated: November 27, 2018)

\begin{abstract}
General forms of the Kähler and superpotenials that lead to consistent low energy broken Supersymmetry originating from $N=1$ Supergravity have been classified and used for model building since more than three decades. We point out the incompleteness of this classification when hidden sector vacuum expectation values are of the order of the Planck mass. Focusing in this paper mainly on the case of minimal Kähler potential, we adopt a rigorous approach that retrieves on the one hand the known forms, and demonstrate on the other hand the existence of a whole set of new forms for the superpotential of which we give a complete classification. The latter forms involve a new type of chiral superfields having the unusual property of belonging neither to the hidden sector nor to the conventional observable sector. Comparing the obtained forms with the conventional ones, we argue how new possibilities for model building can arise, and discuss the gravity mediation of soft as well as additional hard (but parametrically small) Supersymmetry breaking, in the presence of the new type of chiral superfields. In the simplest case, we study the vacuum structure, characterize the masses and couplings of the scalar components to the hidden and observable sectors and discuss briefly the physical role they could play. In the generic case, we estimate the magnitude and possible consequences of the hard breaking of Supersymmetry in terms of the interplay between hidden and visible sectors mass scales.
\end{abstract}

PACS numbers: 04.65.+e, 12.60.Jv, 11.30.Qc

Keywords: Supergravity; Gravity Mediation; Low Energy Supersymmetry; Model Building.

\section{INTRODUCTION}

The experimental discovery of the $\mathcal{H}$-boson at the Large Hadron Collider by the ATLAS [1] and CMS [2] collaborations has completed the building blocks of the standard model (SM) of particle physics. This great experimental success of the SM, the properties of the $\mathcal{H}$-boson having so far proven to be very close to the those of the SM Higgs [3-6], has been accompanied by a forewarning regarding the search for new physics beyond the SM which remains for the time being elusive. In particular the absence of direct experimental signals of low energy broken Supersymmetry (SUSY) may ultimately question, if not its existence, at least the way this symmetry is realized in Nature. Given the theoretical motivations for SUSY, it is of paramount importance to constantly verify the degree of universality on which the low energy models are based since the latter condition the interpretation of the data. Among the various ways to communicate SUSY breaking to the low energy sector, the mediation through gravitational interactions [7-20] in the context of Supergravity, is probably one of the most appealing scenarios as it provides a unique framework encompassing the four fundamental interactions.

In this paper we reconsider the formal consequences of the general requirement any supersymmetric extension of the SM, viewed as an effective low energy remnant of Supergravity, should satisfy in the class of models where hidden sector fields take vacuum expectation values $(V E V S)$ of the order of the Planck mass $\left(\mathcal{O}\left(m_{p \ell}\right)\right)$. In this context we revisit the approach of Soni and Weldon[17] (SW) who analyzed a long time ago the acceptable forms of the superpotential and Kähler potential in that class of models. As these forms encompassed the ones pioneered previously [7-20] and were more general, they have been since relied upon when constructing models where Supersymmetry breaking is mediated by gravitational interactions (see e.g. [21] for a review). It should be noted, though, that having hidden sector VEVs of $\mathcal{O}\left(m_{p \ell}\right)$ became somewhat secondary as the interest shifted towards the celebrated requirement of soft breaking of Supersymmetry that these forms ensure[22]; even more so, since consistent scenarios of gravity mediation that did not assume such big values of the VEVs (see e.g. [23]) were also examined along the line, somewhat lessening the

\footnotetext{
*Electronic address: gilbert.moultaka@umontpellier.fr; corresponding author

${ }^{\dagger}$ Electronic address: michel.rausch@iphc.cnrs.fr

${ }_{\ddagger}^{\ddagger}$ Electronic address: damien.tant@iphc.cnrs.fr
} 
pertinence of the approach adopted initially by SW. However, it could be of some interest to revive such an approach. We see a few reasons for that: (i) the consistency requirement à la SW is nothing else but a tree-level protection of low-scale physics from very large Planck scale effects. In that sense this is just one instance of the tree-level prerequisites when dealing with issues such as the hierarchy problem and the stabilization of largely different scales against quantum effects. (ii) In the presence of $\mathcal{O}\left(m_{p \ell}\right)$ hidden sector VEVs, the general consistency requirements à la SW are found to lead to much richer structures compatible with low energy physics, than previously established. (iii) These structures allow consistently not only soft but also hard breaking contributions. (iv) The approach can be in principle extended to cases where the hidden sector VEVs are much smaller than $\mathcal{O}\left(m_{p \ell}\right)$ but still much larger than other physical scales.

The main goal of the present paper is to demonstrate (ii) and (iii). We provide rigorous proofs that allow a complete classification of the acceptable structures of the superpotential in the case of canonical Kähler potential, and give a few illustrative examples in the case of the general Kähler potential as well. We then discuss on general grounds the possible physical relevance of the novel forms and their associated fields.

The paper is organized as follows: in Section II A we recall the general setting of $N=1$ Supergravity. A summary of the main ingredients of the approach of Ref. [17] is given in Section IIB, as well as a reminder of the general requirement for the consistency of any low energy visible sector of (broken) Supersymmetry in the presence of at least one $\mathcal{O}\left(m_{p \ell}\right)$ VEV. Section III A is devoted to the main results of the paper: we describe our approach and argue why the results of [17] cannot encompass all possible forms of the Kähler potential and superpotential that comply with this requirement. We then give, in the case of the flat Kähler metric, the complete classification of the general forms of the superpotential of $N=1$ Supergravity consistent with low energy physics, yielding not only the conventional ones of [17], but also a whole class of new solutions. Complete details of the rigorous proof of this classification are relegated to appendices A and B 1. A comparison between the features of the new and conventional forms is addressed in section III B. In Section III C and B 2 we exhibit and discuss briefly examples of new solutions in the more involved general Kähler case. A qualitative discussion of the possible implications of the new forms for model building is given in Section IV A. In Section IV B we carry out, in the case of flat Kähler metric, a comparison between the conventional and new forms regarding the gravity mediation of SUSY breaking, and determine the structure of the low energy soft-SUSY breaking terms as well as additional hard breaking terms in presence of the fields that enter the new forms. Section IV C is devoted to an analysis of the simplest new form of the superpotential. There we study the vacuum structure, characterize the masses and couplings of the new fields to the hidden and observable sectors and discuss briefly the physical role they could play. In section IV D we survey briefly the magnitude and possible impact on phenomenology of the hard SUSY breaking terms that are present in the new solution, ending with a few related comments. We conclude in Section V.

\section{II. $\quad N=1$ SUPERGRAVITY, A QUICK REMINDER}

To set the stage we recall first the main ingredients of $N=1$ Supergravity theory and the way it can encompass consistently the low energy models with broken global Supersymmetry. This also serves to define the notations.

\section{A. The general setting}

Consider an $N=1$ Supergravity theory with $k+\ell$ chiral superfields whose scalar field components will be generically denoted by $Z^{I}, I=1, \cdots, k+\ell$. [Throughout the paper we use the following convention: $Z$ denotes the full set of $Z^{I}$ fields, $Z^{\dagger}$ denotes the full set of their complex conjugates $Z^{I^{*}}$, and complex conjugation of a function of these fields is denoted by a bar.] Introducing the Kähler potential $K\left(Z, Z^{\dagger}\right)$ and superpotential $W(Z)$, the $F$-term contribution to the scalar potential takes the form

$$
V_{F}=e^{\frac{K}{m_{p \ell}^{2}}}\left(\mathcal{D}_{I} W K^{I J^{*}} \mathcal{D}_{J} \bar{W}-\frac{3}{m_{p \ell}^{2}}|W|^{2}\right),
$$

with

$$
\mathcal{D}_{I} W=W_{I}+\frac{1}{m_{p \ell}^{2}} K_{I} W
$$


where we define

$$
\begin{aligned}
W_{I} & \equiv \frac{\partial W}{\partial Z^{I}}, \quad\left(\bar{W}_{I^{*}} \equiv \frac{\partial \bar{W}}{\partial Z^{I^{*}}}\right) \\
K_{I} & \equiv \frac{\partial K}{\partial Z^{I}}, \quad\left(K_{I^{*}} \equiv \frac{\partial K}{\partial Z^{I^{*}}}\right)
\end{aligned}
$$

and

$$
K_{I^{*} J}=\frac{\partial^{2} K}{\partial Z^{I^{*}} \partial Z^{J}}
$$

is the Kähler metric, $K^{I J^{*}} \equiv K_{I J^{*}}^{-1}$ its inverse, and $m_{p \ell}$ denotes the reduced Planck mass.

We also recall for later reference the alternative form

$$
V_{F}=m_{p \ell}^{2} e^{\frac{\mathcal{G}}{m_{p \ell}^{2}}}\left(\mathcal{G}_{I} \mathcal{G}^{I J^{*}} \mathcal{G}_{J^{*}}-3 m_{p \ell}^{2}\right)
$$

of Eq. (2.1), where the notational convention is as in Eqs. $(2.4,2.5)$ and $\mathcal{G}\left(Z, Z^{\dagger}\right)$ denotes the generalized Kähler potential

$$
\mathcal{G}=K+m_{p \ell}^{2} \ln \left|\frac{W}{m_{p \ell}^{3}}\right|^{2} .
$$

The gauge sector contributions to the potential

$$
V_{D}=\frac{1}{2}(\operatorname{Re} f)^{\alpha \beta} D_{\alpha} D_{\beta}
$$

where $D_{\alpha}$ denote all the $D$-terms corresponding to the gauge interactions, $f_{\alpha \beta}$ a general gauge kinetic function and $(\operatorname{Re} f)^{\alpha \beta}$ the inverse of its real part as well as all the remaining parts of the Supergravity Lagrangian, including the gauge sector, the superpotential and Kähler geometry dependent scalar-fermion interactions, (see for instance [24]), are not directly relevant to our analysis.

The general setting assumes two distinct sectors: -an observable sector describing the Minimal Supersymmetric Standard Model (MSSM) or any of its extensions including possibly a Grand Unified (GUT) sector -a hidden sector in which local SUSY is assumed to be broken. The notions of visible and hidden sectors are used loosely here. Their physical justification will be made clear in the next section. The chiral superfields split accordingly into $k$ chiral superfields in the observable sector with scalar components denoted by $\Phi^{a}=\left(\Phi^{1}, \cdots\right.$, $\left.\Phi^{k}\right)$, and $\ell$ chiral superfields in the hidden sector with scalar components denoted by $\zeta^{i}=\left(\zeta^{1}, \cdots, \zeta^{\ell}\right)$. The spontaneous breaking of local SUSY occurs when (a combination of) the $\zeta^{i}$ fields develop a vacuum expectation value (VEV) such that

$$
\left\langle F^{I}\right\rangle \neq 0
$$

where

$$
F^{I} \equiv m_{p \ell} e^{\frac{1}{2} \frac{\mathcal{G}}{m_{p \ell}^{2}}} \mathcal{G}^{I J^{*}} \mathcal{G}_{J^{*}}+\cdots
$$

and $I$ labels any of the scalar fields $Z^{I}$; (the dots stand for contributions from the chiral fermionic partners of the $Z^{I}$ or from fermions of vector supermultiplets, whose VEVs will be assumed to vanish in the present study.) The hidden sector $\mathrm{VEV}$ is also assumed to yield a nonvanishing gravitino mass

$$
m_{3 / 2}=\frac{1}{m_{p \ell}^{2}}\left\langle|W| e^{\frac{1}{2} \frac{K}{m_{p \ell}^{2}}}\right\rangle=m_{p \ell}\left\langle e^{\frac{1}{2} \frac{\mathcal{G}}{m_{p \ell}^{2}}}\right\rangle,
$$

while keeping the graviton massless. Although the hidden sector dynamics could be such that $\langle W\rangle \neq 0$ whenever SUSY is broken, this is in general neither necessary nor sufficient unless one imposes a vanishing vacuum energy as well leading to the tree-level relation $\sqrt{3} m_{3 / 2} m_{p \ell}=\left(\left\langle F^{I} F_{I}\right\rangle\right)^{1 / 2} \equiv M_{S}$ where $M_{S}$ denotes the SUSY breaking scale. $^{1}$ The gravitational interactions communicate then SUSY breaking to the visible sector through the generation of soft-SUSY breaking terms triggered by $m_{3 / 2}[7-20]$.

\footnotetext{
1 This remains essentially true when allowing a tiny cosmological constant, albeit issues related to (anti)deSitter spaces in which we do not need to enter in the present study. See for instance [25] and references therein.
} 


\section{B. The Soni-Weldon approach}

It is important to stress that there is in general no guarantee that the above scenario of mediation of SUSY breaking from the hidden sector to the visible sector through gravitational interactions, would not lead to inconsistencies at low energy. Since Supergravity is non-renormalizable and should thus be viewed as an effective description of yet another layer of a more fundamental theory, the Kähler potential and superpotential can a priori be arbitrary functions of the fields and contain arbitrary powers of the Planck mass $m_{p \ell}$. Moreover, in the scenarios we are considering, one expects generically some of the VEVs developed by (some of) the $Z^{I}$ fields to be of order $m_{p \ell}$. But other $Z^{I}$ fields should develop much smaller VEVs so as to set off the electroweak scale and possibly other intermediate scales corresponding to extensions of the SM. Following SW[17], the scalar fields that acquire $\mathcal{O}\left(m_{p \ell}\right)$ VEVs are called hidden sector fields $\zeta^{i}$ responsible for local SUSY breaking, while the visible sector fields $\Phi^{a}$ have much smaller or vanishing VEVs. In order to retrieve consistently the low energy Lagrangian for the visible sector, it is required that:

$$
\begin{aligned}
& \text { all visible sector fields should not appear in the operators of the Lagrangian } \\
& \text { that diverge in the flat limit } m_{p \ell} \rightarrow \infty .
\end{aligned}
$$

From now on we focus on the scalar potential part of the Lagrangian. Near the minimum of the potential, requirement (2.12) implies automatically that the $\zeta$ fields have Planck suppressed couplings to the $\Phi$ fields. Indeed, near the minimum the scalar fields $\operatorname{read} \zeta=\langle\zeta\rangle+\delta \zeta$ and $\Phi=\langle\Phi\rangle+\delta \Phi$, with $|\langle\zeta\rangle| \sim \mathcal{O}\left(m_{p \ell}\right) \gg|\langle\Phi\rangle| \sim|\delta \zeta| \sim|\delta \Phi|$. If, following (2.12), $\Phi$ should be absent from an operator when $\zeta=\langle\zeta\rangle \sim \mathcal{O}\left(m_{p \ell}\right)$, then it obviously remains so by continuity when $\zeta$ is allowed to vary slightly around its $\mathrm{VEV}$ value. This is most easily seen when reasoning on polynomial operators. In particular, operators coupling $\zeta$ and $\Phi$ are forbidden if their dimension is $\leq 4$, and should be suppressed by powers of $m_{p \ell}$ if their dimension is strictly larger than four. It thus appears natural in retrospect to distinguish, as done in the previous section, the notion of hidden sector as comprising $\zeta^{i}$ fields that develop $\mathcal{O}\left(m_{p \ell}\right)$ VEVs from that of observable sector fields $\Phi^{a}$ that have small or vanishing VEVs.

It is important at this point to stress that this definition of hidden and visible sectors is not at odds with the modern and more general use of these terms, namely that their mutual interactions are Planck mass suppressed. Our definition is a special case of the latter and is a sufficient but not necessary condition. As such, while visible sector scalar fields should have VEVs much smaller than $m_{p \ell}$, there should be no ambiguities in the fact that, in contrast, a field having a VEV much smaller than $m_{p \ell}$ is not necessarily in the visible sector. For instance a set of fields $Z^{I}$ related by some unbroken symmetry and developing a VEV of order $m_{p \ell}$ in just one direction would, due to requirement (2.12), have automatically the couplings of all its fields to another sector (that is neutral under this symmetry) suppressed by powers of $m_{p \ell} .^{2}$

We move now to the classification carried out by (SW) [17] of the most general forms of the Kähler potential and the superpotential compatible with the above consistency requirement. In order to treat properly the limit $m_{p \ell} \rightarrow+\infty$ they introduced dimensionless fields in the hidden sector

$$
\zeta^{i}=m_{p \ell} z^{i}
$$

and started off with a Kähler potential $K$ and superpotential $W$ having the general expansions

$$
\begin{aligned}
K\left(Z, Z^{\dagger}\right) & =\sum_{n=0}^{N} m_{p \ell}^{n} K_{n}\left(z, z^{\dagger}, \Phi, \Phi^{\dagger}\right), \\
W(Z) & =\sum_{n=0}^{M} m_{p \ell}^{n} W_{n}(z, \Phi) .
\end{aligned}
$$

Since $W$ and $K$ have respectively mass dimension three and two, the $W_{n}$ and the $K_{n}$ should in general contain other mass scales much smaller than $m_{p \ell}$ (corresponding for instance to some gauge sectors), and/or non canonical powers of $\Phi^{a}$. Note that at low energy the dimensionless hidden sector fields $z$ are by definition $\mathcal{O}(1)$, cf. Eq. (2.13). Then, imposing (2.12) they required the potential to split into two independent sectors: one sector depending exclusively on the hidden sector with possibly positive powers of the Planck mass, the other sector remaining finite with possibly

\footnotetext{
${ }^{2}$ Of course one could also contemplate scenarios where none of the hidden sector VEVs is of order $m_{p \ell}$, (see e.g. [23] for a discussion). We have nothing to say about such scenarios in the present paper, except that a similar approach as the one considered hereafter can be adapted to include several different mass scales.
} 
Planck suppressed hidden/observable sectors interactions, but reducing to the standard flat Supersymmetry form (i.e. the usual $F$ - and $D$-terms) for the observable sector in the large $m_{p \ell}$ limit. Analyzing this strong constraint they arrived at the following special reduced forms of (2.14), (2.15),

$$
\begin{aligned}
K\left(Z, Z^{\dagger}\right) & =m_{p \ell}^{2} K_{2}\left(z, z^{\dagger}\right)+m_{p \ell} K_{1}\left(z, z^{\dagger}\right)+K_{0}\left(z, z^{\dagger}, \Phi, \Phi^{\dagger}\right) \\
W(Z) & =m_{p \ell}^{2} W_{2}(z)+m_{p \ell} W_{1}(z)+W_{0}(z, \Phi)
\end{aligned}
$$

where the $K_{i}$ 's and $W_{i}$ 's are arbitrary functions. The main message here is that the observable sector fields can enter only the $m_{p \ell}$ to the power zero components of Eqs. $(2.14,2.15)$, the otherwise arbitrary $K_{0}, W_{0}$ functions, provided that the exclusively hidden sector components do not have $m_{p \ell}$ powers greater than two. Plugging Eqs. (2.16, 2.17) in Eq. (2.1), one can easily check that these forms are indeed sufficient solutions to satisfy the physical requirement (2.12). However, the claim in [17] was that Eqs. $(2.16,2.17)$ are general enough to exhaust all possible forms consistent with (2.12). In the next section we argue against this claim then revisit the analysis of [17] and indeed exhibit new solutions.

\section{THE GENERAL SOLUTIONS}

We consider here separately the cases of minimal and non-minimal Kähler potentials, but focus mainly on the former giving a thorough discussion and a detailed proof of the classification of all possible solutions.

\section{A. The flat Kähler metric case}

Assuming only canonical kinetic terms for all the chiral superfields, the Kähler potential reads,

$$
K\left(z, z^{\dagger}, \Phi, \Phi^{\dagger}\right)=m_{p \ell}^{2} z^{i *} z^{i}+\Phi^{a *} \Phi^{a}
$$

This form is a special case of Eq. (2.16). Making use of (2.15), the potential (2.1) can be straightforwardly cast in the form

$$
\begin{aligned}
& V_{F}= \exp \left(\frac{Z^{I} Z^{I^{*}}}{m_{p \ell}^{2}}\right) \sum_{0 \leq m \leq M, 0 \leq n \leq M}\left\{m_{p \ell}^{m+n} \frac{\partial W_{m}}{\partial \Phi^{a}} \frac{\partial \bar{W}_{n}}{\partial \Phi^{a *}}+\right. \\
& m_{p \ell}^{m+n-2}\left(\left(\frac{\partial W_{m}}{\partial z^{i}}+z^{i *} W_{m}\right)\left(\frac{\partial \bar{W}_{n}}{\partial z^{i *}}+z^{i} \bar{W}_{n}\right)\right. \\
&\left.+\Phi^{a} \frac{\partial W_{m}}{\partial \Phi^{a}} \bar{W}_{n}+\Phi^{a *} \frac{\partial \bar{W}_{m}}{\partial \Phi^{a *}} W_{n}-3 W_{m} \bar{W}_{n}\right) \\
&\left.+m_{p \ell}^{m+n-4} W_{m} \bar{W}_{n} \Phi^{a *} \Phi^{a}\right\} \\
&=\exp \left(\frac{Z^{I} Z^{I^{*}}}{m_{p \ell}^{2}}\right) \sum_{c=0}^{2 M} V_{M, c}\left[z, z^{\dagger}, \Phi, \Phi^{\dagger}\right] m_{p \ell}^{c}+\mathcal{O}\left(m_{p \ell}^{-1}\right)
\end{aligned}
$$

where in the last equation we collected over all powers of $m_{p \ell}$. [See Eq. (B1) for the explicit expressions of the $V_{M, c}$.] The general requirement (2.12) discussed in the previous section implies that each $V_{M, c}$ with $c \geq 1$ should be functionally independent of $\Phi, \Phi^{\dagger}$. That is:

$$
\frac{\delta}{\delta \Phi^{a}(x)} V_{M, c}\left[z, z^{\dagger}, \Phi, \Phi^{\dagger}\right]=\frac{\delta}{\delta \Phi^{a *}(x)} V_{M, c}\left[z, z^{\dagger}, \Phi, \Phi^{\dagger}\right]=0, \forall a, x, M, c \geq 1 .
$$

As can be seen from Eq. (B1), this constraint turns into a tower of partial differential equations (PDEs) in the unknown superpotential components $W_{n}(z, \Phi)$ of the expansion Eq. (2.15) and their complex conjugates. In order to determine all the superpotential forms that are consistent with Eq. (2.12) one needs to fully solve this tower of PDEs, taking moreover into account the non-trivial constraint of holomorphy of the superpotential.

We are now ready to state the reason for questioning the claim of generality made by the authors of Ref. [17]: rather than following the exhaustive approach we have just described, the focus in Ref. [17] was put exclusively on 
two terms in (3.2), namely, the term containing a linear factor of $\Phi^{a}$ (or $\Phi^{a *}$ ) present in the coefficient of $m_{p \ell}^{m+n-2}$, and the coefficient of $m_{p \ell}^{m+n-4}$ that contains a $\Phi^{a} \Phi^{a *}$ factor. Requiring these two terms to be separately independent of the observable sector fields $\Phi^{a}, \Phi^{a *}$, the authors of [17] were lead to the superpotential given in Eq. (2.17) as the only possibility. While this approach is consistent with requirement (2.12) individually for each one of these two terms, it so happens that the obtained form of the superpotential is sufficient, together with Eq. (3.1), to satisfy (2.12). However, obviously this does not guarantee obtaining all the sufficient and necessary general forms! For one thing, the considered terms can mix with other terms contributing to the same $V_{M, c}\left[z, z^{\dagger}, \Phi, \Phi^{\dagger}\right]$. For another, the various superpotential functions $W_{n}$ are still arbitrary at this stage, and there is a priori no mathematical reason that forbids cancellations from taking place among the various terms in each $V_{M, c}\left[z, z^{\dagger}\right.$, $\left.\Phi, \Phi^{\dagger}\right]$ that would lead to further possibilities consistent with (2.12). This is precisely what the tower of equations (3.4) encodes.

The main purpose of the present paper is to solve completely this tower of equations to clarifiy the seemingly dubious approach of Ref. [17]. This is presented in A and B 1 to which the reader is referred for full details. Here we describe the procedure in a nutshell and summarize the main results of the analysis: starting from the expansion given in Eq.(3.3), each value of $c \geq 1$ implies a PDE, where the unknown function is $W_{n}$ for a given $n$, and the $\Phi^{a}$ 's the variables. To proceed we first solve the PDE corresponding to the highest power of $m_{p \ell}$, that is for $c=2 M$. This gives a general solution for $W_{M}$. Using this solution we then solve the PDE corresponding to $c=2 M-1$ leading to a general solution for $W_{M-1}$. These general solutions are not explicitly holomorphic in the $z^{i}$ and $\Phi^{a}$ fields. Moreover, the structure of $W_{M-1}$ requires distinguishing two types of $\Phi^{a}$ fields, dubbed $\widetilde{\Phi}^{a}$ and $S^{p}$. A detailed and lengthy investigation of the holomorphy requirement leads then to the final general forms of $W_{M}$ and $W_{M-1}$, see Eqs. (B48, B49). If the expansion in Eq. (2.15) is truncated at $M=1$, then there are no further PDEs to solve. If $M>1$, examination of the PDEs corresponding to $c=2 M-2,2 M-3, \ldots$ allows to determine the general forms of $W_{M-2}, W_{M-3}, \ldots$ but shows that the expansion in Eq. (2.15) should be truncated at $M=2$. Moreover, the general forms in the latter case do not allow for fields of the $S^{p}$ type. We summarize the results as follows:

- if the series (2.15) extends beyond $M=2$, with $W_{n}(z, \Phi)$ non vanishing functions for $n \geq 3$, then there exist no solutions for Eq. (2.15) satisfying (2.12).

- if the series $(2.15)$ is truncated at $M=2$, i.e. $W_{n}(z, \Phi)$ are possibly non vanishing only when $n<3$, there exist two general solutions satisfying (2.12),

(1) Soni-Weldon:

$$
W(\zeta, \Phi)=m_{p \ell}^{2} W_{2}(z)+m_{p \ell} W_{1}(z)+W_{0}(z, \Phi),
$$

where the $W_{1,2}$ are arbitrary holomorphic functions of the $z^{i}$ fields and $W_{0}$ an arbitrary holomorphic function of the $z^{i}$ and $\Phi^{a}$ fields, corresponding to the solution found in [17]. We will refer to this solution as SWS.

(2) Non-Soni-Weldon: in this case $W_{2} \equiv 0$ and the visible sector fields $\Phi$ should be split into two types denoted $\widetilde{\Phi}$ and $S$. Moreover, different solutions entail different partitions of the set of $S$ fields. The general superpotential reads,

$$
W(\zeta, \Phi)=m_{p \ell} W_{1}(z, S)+W_{0}(z, S, \widetilde{\Phi})
$$

with

and

$$
W_{1}(z, S)=W_{1,0}(z)+\sum_{p \geq 1}^{P} W_{1, p}(z) \sum_{s \geq 1}^{n_{p}} \mu_{p_{s}}^{*} S^{p_{s}}
$$

$$
W_{0}(z, S, \widetilde{\Phi})=\sum_{q \geq 1}^{k_{1}} W_{0, q}(z) S^{q}+\Xi\left(\ldots, \mathcal{U}_{S}^{p p_{s}} \ldots ; \ldots, \widetilde{\Phi}^{a}, \ldots ; \ldots, z^{i}, \ldots\right),
$$

where $W_{1,0}(z), W_{1, p}(z)$ and $W_{0, p}(z)$ are arbitrary holomorphic functions of the $z^{i}$ fields, $\Xi$ an arbitrary function of all its entries and holomorphic in the $z^{i}, \widetilde{\Phi}^{a}, S^{q}$ fields, and we define

$$
\mathcal{U}_{S}^{p p_{s}} \equiv \xi_{p_{s}}(z) S^{p_{s}}-\xi^{p_{s}}(z) S^{p_{1}} \text {, with } p=1, \ldots, P \text {, and } s=1, \ldots, n_{p},
$$

the two sets of functions $\xi^{p_{s}}(z)$ and $\xi_{p_{s}}(z)$ denoting arbitrary holomorphic functions of the $z^{i}$ fields satisfying

$$
\mu_{p_{s}} \xi_{p_{s}}(z)=\mu_{p_{1}} \xi^{p_{s}}(z) .
$$


The $\mu_{p_{s}}$ 's entering Eq. (3.10), and their complex-conjugate entering Eq. (3.7), denote arbitrary nonvanishing complex-valued $k_{1}$ constants, $k_{1}$ being the total number of $S$ fields, while $P$ and $n_{p}$ are arbitrary (positive) integers satisfying

$$
k_{1}=\sum_{p \geq 1}^{P} n_{p} .
$$

The latter define a given partition of the set of $S$ fields into $P$ subsets labeled by $p=1, \ldots, P$, where $n_{p}$ denotes the number of elements in the $p^{t h}$ set. See also the discussion following Eq. (B47) and Eq. (B52). We will refer to this new solution as non-Soni-Weldon (NSWS). It represents the central result of the paper.

\section{B. Comparing SWS and NSWS}

The two classes of solutions are different in various ways, and NSWS is clearly not a special case of SWS. We list here some of the salient features:

1. The SWS confines the dependence on the observable sector fields $\Phi$ to the $m_{p \ell}$ independent component of the superpotential. In contrast, NSWS requires to make a distinction between two subsets of the observable sector, the $\widetilde{\Phi}$-type and the $S$-type fields.

2. Both $\widetilde{\Phi}$ and $S$ have the properties of the observable sector in the sense that they satisfy $(2.12)$.

3. The $\widetilde{\Phi}$-type fields have a status similar to that of $\Phi$ in SWS, they enter only the $m_{p \ell}$ independent component of the superpotential through the arbitrary function $\Xi$. All the conventional implementations of the SUSY extensions of the SM in the observable sector (from [7, 8], [14], onwards) that rely on SWS, can thus be carried over unchanged to the $\widetilde{\Phi}$ sector of NSWS.

4. The presence of the $S$ fields in the NSWS case offers new possibilities. A striking difference with SWS is that the $S$ fields, even though observable, appear in the term of the superpotential that is proportional to $m_{p \ell}$. This seemingly counter-intuitive result comes, however, at the price of requiring linearity in the $S$-type in $W_{1}$, and in $W_{0}$, the arbitrary dependence exclusively through the combinations $\mathcal{U}_{S}^{p p_{s}}$ in $\Xi$, modulo an extra linear piece in the $S$-type. It should be clear that the set of all possible general functions of $\mathcal{U}_{S}^{p p_{s}}$ does not contain all the set of possible general functions of the $S$ fields.

5. The above point is the key issue distinguishing the $\widetilde{\Phi}$-type from the $S$-type. It is easy to see that this difference is triggered by the nonvanishing $\mu_{p_{s}}$ constants; if a given $S^{p_{s}}$ field is functionally absent from $W_{1}$, that is if $\mu_{p_{s}}=0$, then the corresponding $\mathcal{U}_{S}^{p p_{s}}$ reduces to $\xi_{p_{s}}(z) S^{p_{s}}$ as a consequence of Eq. (3.10). The function $\Xi$ becomes arbitrary in $S^{p_{s}}$ so that the latter field has the same status as the $\widetilde{\Phi}$-type fields and becomes part of the latter set. On the other hand, a given $S^{p_{s}}$ can be present in $W_{1}$ and absent from $\Xi$ and still remain part of the $S$-type set. Indeed this would correspond to a choice of the partition in $P$ subsets with $\left\{S^{p_{s}}\right\}$ being one element of this partition.

6. An important peculiarity of the NSWS is that direct couplings with arbitrary strength between the $\widetilde{\Phi}$ and $S$ sectors (as well as within the $S$ sector itself) in the superpotential, are allowed only if there exist two or more different $S$ fields in the theory. This is again a direct consequence of the fact that the $S$ dependence in $\Xi$ enters exclusively through the $\mathcal{U}_{S}^{p p_{s}}$ combinations.

7. With at least two $S$-type fields in the theory, the $\Xi$ part of the superpotential will always contribute F-flat directions to the potential, defined by $\mathcal{U}_{S}^{p p_{s}}=0$. These directions are however generically lifted by the mandatory linear $S$ dependence in $W_{1}$, and possibly the linear dependence in $W_{0}$. We come back to this point at the end of Section IV C.

8. If, in contrast, there is only one single $S$-type field in the theory, then it appears only linearly in the superpotential and, as we show in Section IV C, has Planck mass suppressed couplings to both the hidden and the remaining part of the observable sectors.

9. Finally, note that there is only one configuration where both SWS and NSWS reduce to the same form: if $\mu_{p_{s}}=0, \forall p_{s}$, in Eq. (3.7) then the relation (3.10) becomes trivial and $\Xi$ an arbitrary function of the $S$ fields. The fields $\widetilde{\Phi}$ and $S$ are then formally indistinguishable and $W_{0}(z, S, \widetilde{\Phi})$ corresponds to $W_{0}(z, \Phi)$ of SWS. The two classes of solutions become then equivalent if $W_{2}$ in Eq. (3.5) is a vanishing function of $z$. 
We will see in Section IV how the above formal properties come into play in relation with model building.

\section{The general Kähler case}

This section is somewhat outside the scope of the present paper. It aims at pointing out that the existence of new forms as found in Section III A is not a peculiarity of the minimal Kähler assumption Eq. (3.1).

We exhibit here two non-Soni-Weldon solutions in the case of non minimal Kähler. Starting from the general superpotential and Kähler potential with $M=N=2$ in Eqs. $(2.14,2.15)$,

$$
\begin{aligned}
K\left(z, z^{\dagger}, \Phi, \Phi^{\dagger}\right) & =m_{p \ell}^{2} K_{2}\left(z, z^{\dagger}, \Phi, \Phi^{\dagger}\right)+m_{p \ell} K_{1}\left(z, z^{\dagger}, \Phi, \Phi^{\dagger}\right)+K_{0}\left(z, z^{\dagger}, \Phi, \Phi^{\dagger}\right), \\
W(z, \Phi) & =m_{p \ell}^{2} W_{2}(z, \Phi)+m_{p \ell} W_{1}(z, \Phi)+W_{0}(z, \Phi),
\end{aligned}
$$

we find that the consistency requirement (2.12) is satisfied if:

- $W_{1}, K_{1}$ are vanishing functions,

- $W_{0}, K_{0}$ are arbitrary functions,

- $W_{2}, K_{2}$ are arbitrary functions that should depend explicitly on $\Phi, \Phi^{\dagger}$, subject to a no-scale-like condition [26-29]

$$
\partial_{I} \mathcal{G}_{2}\left(\frac{\partial^{2} \mathcal{G}_{2}}{\partial Z^{I} \partial Z^{J^{*}}}\right)^{-1} \partial_{J^{*}} \mathcal{G}_{2}=3, \text { with } \mathcal{G}_{2}=K_{2}+\ln \left|\frac{W_{2}}{m_{p \ell}}\right|^{2},
$$

or alternatively if:

- $W_{2}$ is a vanishing function,

- $W_{0}, W_{1}, K_{0}, K_{1}, K_{2}$ are arbitrary functions that should depend explicitly on $\Phi, \Phi^{\dagger}$.

We stress the difference with the general form given in [17] where the functions $K_{1}, K_{2}, W_{1}, W_{2}$, although arbitrary, had to be independent of the observable fields, $\Phi, \Phi^{\dagger}$, while for instance in the first solution given above $K_{2}$ and $W_{2}$ must depend on these fields provided that $K_{1}$ and $W_{1}$ are vanishing. See B 2 for more details.

\section{TOWARDS MODEL BUILDING AND PHENOMENOLOGY FROM THE NSWS}

We return here to the NSWS in the minimal Kähler case to discuss possible realizations of the role the $S$-type sector can play in model building.

\section{A. General considerations}

This section is devoted to a non-exhaustive broad-brush discussion of the various possibilities. So far we have ignored features related to gauge or global symmetries. As already stated in Section III B, the $\widetilde{\Phi}^{a}$ fields of the NSWS can play the same role as the $\Phi^{a}$ fields of the SWS for model building. The $\Xi$ function can thus contain the superpotential of the MSSM or any other SUSY extension of the SM by implementing the corresponding fields and their quantum numbers in the $\widetilde{\Phi}^{a}$ set. Can the $S$ fields play the same role? There is an obstruction because of the linear terms in $S$ in $W_{1}$ : since the hidden sector fields are assumed to be neutral under the SM gauge groups, all the $W_{1, p}$ are singlets, thus all the $S$ fields must be singlets under these same groups so as to ensure the invariance of $W_{1}$. The $S$-type sector is thus particularly suited to non-minimal SUSY extensions with SM singlet fields. The existence of the NSWS can even be seen as a motivation for such extensions. The next to minimal MSSM (NMSSM) [30, 31] is the first example that comes to mind. However, as stated in point 6 . of Section (III B), a realization in the $S$ sector should have at least two different gauge singlet fields, and thus corresponds to extensions of the NMSSM itself. Such extra singlets could be welcome for various reasons (see e.g. [32] for a review). But it should be kept in mind that their embedding as $S$-type fields is physically not equivalent to their conventional embedding as $\widetilde{\Phi}$-type fields. Among the usual terms of the NMSSM Higgs superpotential,

$$
\lambda S H_{u} \cdot H_{d}, \xi_{F} S, \frac{1}{2} \mu^{\prime} S^{2}, \frac{1}{3} \kappa S^{3},
$$


(we use the notation of [32]), only the "tadpole" can preserve its form, while in all the others the field $S$ should be replaced by, at least, one combination of the form $\mathcal{U}_{S}^{a b}$ :

$$
\lambda \mathcal{U}_{S}^{a b} H_{u} \cdot H_{d}, \xi_{F}^{a} S^{a}+\xi_{F}^{b} S^{b}, \frac{1}{2} \mu^{\prime}\left[\mathcal{U}_{S}^{a b}\right]^{2}, \frac{1}{3} \kappa\left[\mathcal{U}_{S}^{a b}\right]^{3},
$$

where $S^{a}$ and $S^{b}$ denote two independent $S$-type fields. As can be seen from Eqs. $(3.6,3.8)$ the tadpole parameters $\xi_{F}^{a}, \xi_{F}^{b}$ have now their origin in the hidden sector of Supergravity. They are given respectively by $\left\langle m_{p \ell} W_{1, a}(z)+W_{0, a}(z)\right\rangle$ and $\left\langle m_{p \ell} \mu_{b}^{*} W_{1, a}(z)+W_{0, b}(z)\right\rangle$ at low energies where the hidden sector degrees of freedom are frozen at their VEVs and we assumed the normalisation $\mu_{a}=1$. Similarly, $\mathcal{U}_{S}^{a b}=\xi_{b}(\langle z\rangle)\left(S^{b}-\mu_{b} S^{a}\right)$, cf. Eq. (3.9) where we have used Eq. (3.10) and the same normalisation. More generally, perturbing around $\langle z\rangle$ one has a definite structure for the interaction between the hidden and the observable sector through the singlet fields in this extended version of the NMSSM. Another issue is related to the so-called 'tadpole problem'. In the NSWS case the two parameters $\xi_{F}^{a}, \xi_{F}^{b}$ have contributions proportionnal to the Planck mass. However by construction in the NSWS such a term should vanish in the potential.

Another possibility for model building is to consider $S$-type fields that are non-singlets under symmetries involving the hidden sector while remaining SM singlets. For instance a subset of these fields could be charged under hidden abelian or non-abelian gauge groups. If direct couplings of such fields to the observable sector are forbidden through the $\Xi$ function then whatever physics occuring in the hidden sector would be communicated to the visible sector only by gravitionally suppressed effects. This would be necessarily the case if there is only one single $S$-type multiplet charged under a given hidden gauge group $\mathfrak{G}$. Indeed in this case the full set of $S^{q}$ fields spans the components of a multiplet $\mathbf{S}$ of a given representation of $\mathfrak{G}$. In order for the term $\sum_{p \geq 1}^{P} W_{1, p}(z) \sum_{s \geq 1}^{n_{p}} \mu_{p_{s}}^{*} S^{p_{s}}$ in Eq. (3.8) to be gauge invariant, $W_{1, p}(z)$ should be the $p^{\text {th }}$ component of the conjugate representation and simultaneously the sum $\sum_{s \geq 1}^{n_{p}}$ should reduce to only one term, i.e. $n_{p}=1$ for each $p$. This corresponds to the singleton partition of the set of $S^{q}$ fields made of the $k_{1}$ subsets $\left\{S^{q}\right\}$ with $k_{1}=P$ being equal to the dimension of the considered group representation. It follows that all $\mathcal{U}_{S}^{p p_{s}}$ are vanishing and thus no direct coupling can be constructed between the $S$-type and $\widetilde{\Phi}$-type fields in $\Xi$, (see also item five of Section (IIIB)). Note that the same conclusion holds trivially when $\mathfrak{G}$ is abelian, since in this case to have a single multiplet means to have one single $S$ field.

Direct coupling between the $S$-type and $\widetilde{\Phi}$-type fields requires at least two multiplets $\mathbf{S}_{1}, \mathbf{S}_{2}$ belonging to the same group representation. The gauge invariance of the sum just discussed enforces a unique choice for the partition of the full set of $S$ fields. Each element of the partition should now be of the form $\left\{S_{1}^{r}, S_{2}^{r}\right\}$, the label $r$ spanning the components of the representation under consideration. With the correspondence $p_{1} \rightarrow(r, 1), p_{n_{p}} \rightarrow(r, 2)$, the sum reads

$$
\sum_{r \geq 1}^{N} W_{1, r}(z)\left(\mu_{(r, 1)}^{*} S_{1}^{r}+\mu_{(r, 2)}^{*} S_{2}^{r}\right),
$$

where $N=k_{1} / 2$ is the dimension of the representation. One can also construct the combinations $\mathcal{U}_{S}$, cf. Eqs. (3.9, 3.10) to write, adapting slightly the notation,

$$
\mathcal{U}_{S_{12}}^{r}=\xi_{r}(z)\left(\mu_{(r, 1)} S_{2}^{r}-\mu_{(r, 2)} S_{1}^{r}\right) .
$$

It is noteworthy that in general the constant factors $\mu_{(r, 1)}, \mu_{(r, 2)}$ cannot be absorbed in a redefinition of the $S$ fields as they multiply them differently in the above expressions. Gauge invariance, however, requires them to be equal. Thus choosing $\mu_{(r, 1)}=\mu_{(r, 2)}$ gives now the freedom to reabsorb this common factor in a redefinition of the functions $W_{1, r}(z)$ and $\xi_{r}(z)$. Requiring furthermore $W_{1, r}(z)$ and $\xi_{r}(z)$ to be the components of the conjugate representations, the term in Eq. (4.3) becomes gauge invariant, as well as the sum of the $\mathcal{U}_{S}$ combinations

$$
\mathcal{U}_{S_{12}} \equiv \sum_{r \geq 1}^{N} \mathcal{U}_{S_{12}}^{r} .
$$

The latter constitutes an interesting building block for the construction of new extensions. For instance all the terms 
listed in Eq. (4.2) can be simply generalized by the replacements

$$
\begin{aligned}
\mathcal{U}_{S}^{a b} & \rightarrow \mathcal{U}_{S_{12}}, \\
\xi_{F}^{a} S^{a}+\xi_{F}^{b} S^{b} & \rightarrow \sum_{r \geq 1}^{N}\left(\xi_{F_{1}}^{r}(z) S_{1}^{r}+\xi_{F_{2}}^{r}(z) S_{2}^{r}\right),
\end{aligned}
$$

where

$$
\xi_{F_{1}}^{r}(z)=m_{p \ell} W_{1, r}(z)+W_{0, r}^{(1)}(z), \xi_{F_{2}}^{r}(z)=m_{p \ell} W_{1, r}(z)+W_{0, r}^{(2)}(z) .
$$

Recall that the arbitrary functions $W_{0, r}^{(1)}(z), W_{0, r}^{(2)}(z)$, that correspond to the functions $W_{0, q}(z)$ of Eq. (3.8), are not mandatory for the $S$-type characterization, and can be chosen vanishing contrary to the $W_{1, r}(z)$ functions. The obtained terms in the superpotential provide an unconventional general structure for the interaction between the hidden and visible sectors in the realm of singlet extensions of the MSSM that complies with the general consistency requirement for low energy Supergravity. Moreover, when the hidden sector fields acquire VEVs the replacement $z \rightarrow\langle z\rangle$ generates dynamically the low energy superpotential terms of the MSSM singlet extensions that are invariant under the SM gauge groups and break spontaneously the hidden gauge group $\mathfrak{G}$. From this perspective the NMSSM extension corresponding to the terms given in Eq. (4.2) can be interpreted as a special case of a spontaneously broken $U(1)$ factor in $\mathfrak{G}$.

Another interesting possibility arises when considering secluded sectors such as in gauge mediation [33-43], or dark sectors [44]. These sectors are still considered as observable with respect to the hidden sector of Supergravity, but are not directly coupled to the SM observable sector. Some of the $S$-type fields can now be charged under the symmetry groups of these sectors, thus linking them naturally to the hidden sector at least through the mandatory linear terms in the superpotential Eq. (3.7). Moreover, in contrast with the case of the observable sector, some of the $S$-type fields can now play the role of the matter superfields in these sectors. For instance, in a dark copy of the SM the NSWS structure of the superpotential $\Xi$ predicts the existence of at least two 'quark' and 'lepton' families. Also a dark or secluded Yukawa sector should necessarily be of the form

$$
\left(\mathcal{U}_{S}^{a b}\right)_{L} \cdot H\left(\mathcal{U}_{S}^{a b}\right)_{R}
$$

where $\left(\mathcal{U}_{S}^{a b}\right)_{L, R}$ denote doublets and singlets of the dark $S U(2)$, thus predicting naturally nonzero off-diagonal Yukawa couplings.

\section{B. Supergravity mediated SUSY breaking}

In this section we compare explicitly the usual gravity mediation SUSY breaking assuming the superpotential to be a direct sum $[7,8]$ of the hidden and visible sectors functions in the SWS case, to the gravity mediation in the NSWS case with a similar direct sum assumption. The first important general difference lies in the fact that the conventional SWS allows a nonzero $W_{2}$ term in the superpotential, while the NSWS forbids it allowing at most a $W_{1}$ term. This implies typically that after SUSY breaking the gravitino mass Eq. (2.11) is suppressed by a factor $M / m_{p \ell}$ in the NSWS case as compared to the SWS case, where $M\left(<m_{p \ell}\right)$ denotes the largest mass scale other than the Planck scale present in the theory, e.g. a GUT scale. Another important difference comes from the structure of the ensuing SUSY breaking parameters.

\section{The SWS case}

Let us first recall how one arrives at the well-known structure of the potential when positing a direct sum superpotential in the SWS case. We start from

$$
W(\zeta, \Phi)=h(\zeta)+g(\Phi)
$$

corresponding to Eq. (3.5) with

$$
W_{2}(z)=m_{p \ell}^{-2} h(\zeta) \equiv M_{2} \omega_{2}(z), W_{1}(z)=0, \text { and } W_{0}(z, \Phi)=g(\Phi),
$$


where $M_{2}$ is a mass scale much smaller than $m_{p \ell}$. Assuming a minimal Kähler, the $F$-term contribution to the potential, Eq. (2.1), now reads

$$
V_{F}=e^{\frac{\left|\zeta^{i}\right|^{2}+\left|\Phi^{a}\right|^{2}}{m_{p \ell}^{2}}}\left(\left|h_{i}+\frac{\zeta^{i *}}{m_{p \ell}^{2}} W\right|^{2}+\left|g_{a}+\frac{\Phi^{a *}}{m_{p \ell}^{2}} W\right|^{2}-\frac{3}{m_{p \ell}^{2}}|W|^{2}\right),
$$

with the notation as in Eq. (2.3). When the hidden sector fields $\zeta^{i}$ acquire a VEV the gravitino mass Eq. (2.11) is expressed as

$$
m_{3 / 2}=\frac{1}{m_{p \ell}^{2}}\left\langle|h(\zeta)| e^{\frac{1}{2} \frac{\left|\zeta^{i}\right|^{2}}{m_{p \ell}^{2}}}\right\rangle=M e^{\frac{1}{2}\left\langle\left|z^{i}\right|^{2}\right\rangle},
$$

where we define $M \equiv M_{2}\left\langle\omega_{2}(z)\right\rangle$. To obtain the low energy form of the potential $V_{F}$ in the flat SUSY limit we make the substitution $z \rightarrow\langle z\rangle$ and retain the leading terms in the flat limit $m_{p \ell} \rightarrow \infty$ with $m_{3 / 2}$ fixed. Adopting slightly modified notations from the conventional ones [19], we write

$$
\begin{aligned}
& \left\langle\zeta^{i}\right\rangle=m_{p \ell}\left\langle z^{i}\right\rangle \equiv m_{p \ell} b_{i} \\
& \langle h\rangle \equiv M m_{p \ell}^{2},\left\langle h_{i}\right\rangle=\left\langle\frac{\partial h}{\partial \zeta^{i}}\right\rangle \equiv a_{i}^{*} M m_{p \ell} .
\end{aligned}
$$

Plugging these back in Eq. (4.11) and keeping the leading operators, we retrieve the well-known low energy softly broken SUSY potential [7, 8], [19], with SUSY breaking mass terms for the $\Phi^{a}$ fields and couplings:

$$
\begin{array}{r}
V_{\mathrm{LE}}^{\mathrm{SWS}}=\left|\frac{\partial \widehat{g(\Phi)}}{\partial \Phi^{a}}\right|^{2}+m_{3 / 2}^{2}\left|\Phi^{a}\right|^{2}+m_{3 / 2}\left((A-3) \widehat{g(\Phi)}+\Phi^{a} \frac{\partial \widehat{g(\Phi)}}{\partial \Phi^{a}}+\text { h.c. }\right) \\
+\mathcal{O}\left(m_{p \ell}^{-2}\right)
\end{array}
$$

where

$$
A \equiv \sum_{i} b_{i}^{*}\left(a_{i}+b_{i}\right)
$$

a factor $\exp \frac{1}{2}\left|b_{i}\right|^{2}$ has been absorbed in the hatted $g$, and we have omitted an additive constant with positive powers of $m_{p \ell}$, originating from the VEVed purely hidden sector (see footnote 4 ). Recall that choosing a direct product potential [14] would have given a similar result albeit with the special relation $A=3$.

\section{The NSWS case}

To compare with the NSWS case, we assume for the latter a minimal Kähler potential,

$$
K(z, S, \widetilde{\Phi})=m_{p \ell}^{2} z^{i} z^{i *}+S^{p} S^{p *}+\widetilde{\Phi}^{a} \widetilde{\Phi}^{a *},
$$

where we distinguish explicitly the $S^{p}$ fields, and the following direct sum superpotential:

$$
W(\zeta, S, \widetilde{\Phi})=h\left(\zeta^{i}, S^{q}\right)+g\left(S^{q}, \widetilde{\Phi}^{a}\right)
$$

with

$$
h\left(\zeta^{i}, S^{q}\right)=m_{p \ell}\left(W_{1,0}\left(z^{i}\right)+W_{1,1}\left(z^{i}\right) \sum_{q \geq 1}^{k_{1}} \mu_{q}^{*} S^{q}\right)
$$

and

$$
g\left(S^{q}, \widetilde{\Phi}^{a}\right)=\Xi\left(\ldots, \mu_{1} S^{q}-\mu_{q} S^{1}, \ldots, \widetilde{\Phi}^{a}, \ldots\right) .
$$

This corresponds to choosing the trivial partition in the $S$-type sector, i.e. $W_{1, q}(z):=W_{1,1}(z), \forall q$, and we take for simplicity $W_{0, q}(z):=0$. Note that by construction the NSWS solutions do not allow the separation of the $S$-type fields from the hidden sector fields in the form of a direct sum. By taking $\Xi$ independent of the hidden sector our 
choice is the closest possible to such a separation. (However a separation in the form of a direct product is possible and comes naturally with the present choice of singleton partition, provided that $W_{1,0}(z):=0$ and $\Xi$ is appropriately chosen as a direct product.)

Defining

$$
W_{1,0}(z) \equiv M_{10}^{2} \omega_{10}(z), W_{1,1}(z) \equiv M_{11} \omega_{11}(z),
$$

where, as in the previous case, $M_{10}$ and $M_{11}$ denote some physical mass scales much smaller than $m_{p \ell}$, and

$$
M \equiv\left|M_{10}^{2}\left\langle\omega_{10}(z)\right\rangle+M_{11}\left\langle\omega_{11}(z)\right\rangle \sum_{q} \mu_{q}^{*}\left\langle S^{q}\right\rangle\right|^{\frac{1}{2}},
$$

the gravitino mass is given by

$$
m_{3 / 2}=\frac{1}{m_{p \ell}^{2}}\left\langle|h(\zeta, S)| e^{\frac{1}{2} \frac{\mid \zeta^{i}{ }^{2}}{m_{p \ell}^{2}}}\right\rangle=\frac{M^{2}}{m_{p \ell}} e^{\frac{1}{2}\left|b_{i}\right|^{2}},
$$

where we neglected the VEV of the $S$ fields in the exponential, as well as possible contributions from the visible sector Eq. (4.20) since they are typically suppressed by one power of $m_{p \ell}$ as compared to the hidden sector contribution. ${ }^{3}$ (To keep the discussion at the generic level, we do not consider possible unabsorbable complex phases in the various VEVs.) It is noteworthy that in the present case the mass scale $M$ can get contributions from the VEV of the $S$-type fields, which can even be the leading effects, depending on the relative magnitudes of the mass scales $M_{10}, M_{11}$ and the dimensionless numbers $\left\langle\omega_{10}(z)\right\rangle,\left\langle\omega_{11}(z)\right\rangle$. One can thus consider model settings where the $S$ fields trigger the gravity mediation of SUSY breaking to the visible sector, even though those fields are not part of the hidden sector. More generally, when the mass scale $M \ll m_{p \ell}$ has a comparable magnitude to that of the SWS case, the gravitino mass is expected to be typically smaller due to the Planck scale suppression in Eq. (4.23) as compared to Eq. (4.12). To proceed, let us define

$$
\begin{aligned}
& \langle h\rangle \equiv M^{2} m_{p \ell},\left\langle\frac{\partial h}{\partial \zeta^{i}}\right\rangle=\left\langle h_{i}\right\rangle \equiv a_{i}^{*} M^{2}, \\
& \left\langle\frac{\partial h}{\partial S^{q}}\right\rangle=\left\langle h_{q}\right\rangle=m_{p \ell} M_{11}\left\langle\omega_{11}(z)\right\rangle \mu_{q}^{*} \equiv a_{q}^{*} M m_{p \ell} .
\end{aligned}
$$

Note the different mass powers in $\langle h\rangle$ and $\left\langle h_{i}\right\rangle$ between Eq. (4.14) and Eq. (4.24). We can now consider the low energy potential $V_{F}$ in the SUSY flat limit by taking as previously the limit $m_{p \ell} \rightarrow \infty$ with fixed $m_{3 / 2}$ and making the substitution $\zeta \rightarrow\langle\zeta\rangle$ to which we add the substitution $S^{q} \rightarrow S^{q}+\left\langle S^{q}\right\rangle$ thus allowing for nonvanishing $S$-fields VEVs. The latter shift leads to the relation

$$
h_{i}=\left\langle h_{i}\right\rangle+M_{11}\left\langle\frac{\partial \omega_{11}(z)}{\partial z^{i}}\right\rangle \sum_{q} \mu_{q}^{*} S^{q} \equiv a_{i}^{*} M^{2}+a_{i}^{\prime *} M \sum_{q} \mu_{q}^{*} S^{q},
$$

which also defines the parameters $a_{i}^{\prime *}$. Plugging back in Eq. (4.11) the various pieces of the superpotential Eqs. (4.18, $4.19,4.20)$ and their derivatives, adding the $S$-fields contribution $\left|\frac{\partial W}{\partial S^{q}}+\frac{S^{q *}}{m_{p \ell}^{2}} W\right|^{2}$, and taking into account Eqs. (4.13, $4.24,4.25,4.26$ ), we find (a sum over all indices being understood),

$$
\begin{aligned}
V_{\mathrm{LE}}^{\mathrm{NSWS}}= & \left|\frac{\partial \widehat{\Xi}}{\partial \widetilde{\Phi}^{a}}\right|^{2}+\left|\frac{\partial \widehat{\Xi}}{\partial S^{q}}\right|^{2}+m_{3 / 2}^{2}\left(\left|\widetilde{\Phi}^{a}\right|^{2}+\left|S^{q}+\left\langle S^{q}\right\rangle\right|^{2}\right)\left|1+A^{(S)}\right|^{2} \\
& +m_{3 / 2}\left(\left(A-3+\left\langle A^{(S)}\right\rangle+\left(\left|b_{i}\right|^{2}-2\right) A^{(S)}+b_{i}^{*} A_{i}^{\prime(S)}\right) \widehat{\Xi}\right. \\
& \left.+\left(1+A^{(S)}\right) \widetilde{\Phi}^{a} \frac{\partial \widehat{\Xi}}{\partial \widetilde{\Phi}^{a}}+\left(1+A^{(S)}\right)\left(S^{q}+\left\langle S^{q}\right\rangle\right) \frac{\partial \widehat{\Xi}}{\partial S^{q}}+\text { h.c. }\right) \\
& +e^{\left|b_{i}\right|^{2}} M^{2} \mathcal{A}_{q r} S^{q} S^{r *}+e^{\left|b_{i}\right|^{2}} M^{3}\left(\left(\left(A+\left\langle A^{(S)}\right\rangle-2\right) a_{q}^{*}+A^{\prime} \mu_{q}^{*}\right) S^{q}+\text { h.c. }\right) \\
& +\mathcal{O}\left(m_{p \ell}^{-2}\right),
\end{aligned}
$$

\footnotetext{
3 This approximation is however not essential here and depends on the actual magnitudes of the physical scales involved. We come back to this point in section IV D.
} 
where we have again omitted additive constants to the potential originating from the VEVed hidden sector or from VEVs of the coupled hidden and $S$ sectors ${ }^{4}$, and defined $A$ as in Eq. (4.16) and

$$
\begin{aligned}
A^{\prime} & \equiv \sum_{i} a_{i}^{\prime *}\left(a_{i}+b_{i}\right), \\
A^{(S)} & \equiv \frac{1}{M} \sum_{q} a_{q} S^{q *}=\frac{M_{11}}{M^{2}}\left\langle\omega_{11}(z)\right\rangle^{*} \sum_{q} \mu_{q} S^{q^{*}}, \\
A_{i}^{\prime(S)} & \equiv \frac{1}{M} a_{i}^{\prime} \sum_{q} \mu_{q} S^{q *}=\frac{M_{11}}{M^{2}}\left\langle\frac{\partial \omega_{11}(z)}{\partial z^{i}}\right\rangle^{*} \sum_{q} \mu_{q} S^{q^{*}}, \\
\mathcal{A}_{q r} & \equiv \sum_{i}\left(b_{i} a_{q}+a_{i}^{\prime} \mu_{q}\right)^{*}\left(b_{i} a_{r}+a_{i}^{\prime} \mu_{r}\right)-a_{q}^{*} a_{r} .
\end{aligned}
$$

A few comments are in order here. Note first that dangerous Planck enhanced contributions involving the $S$ fields and originating from the crossed terms in $\left|\frac{\partial W}{\partial S^{q}}+\frac{S^{q *}}{m_{p \ell}^{2}} W\right|^{2}$ have, as expected, canceled out as a result of the interplay between the specific forms of the $S$-dependent terms in Eqs. $(4.19,4.20)$. This confirms that the $S$-type fields are part of the visible sector in the sense of (2.12). We also retrieve the usual SUSY preserving contributions, the first two terms of Eq. (4.27). Second, the soft SUSY breaking contribution to the masses of the $\widetilde{\Phi}$-type and $S$-type fields, proportional to $m_{3 / 2}^{2}$ in Eq. (4.27), are the same as in $V_{\mathrm{LE}}^{\mathrm{SWS}}$, Eq. (4.15), but there are new interaction terms with the $S$-type fields due to the presence of $A^{(S)}$ that we will discuss separately at the end of this subsection. Third, the SUSY breaking terms proportional to $m_{3 / 2}$ have now a much richer structure in comparison with $V_{\mathrm{LE}}^{\mathrm{SWS}}$ : apart from the presence of new interaction terms with the $S$ fields that we will also discuss separately at the end of this subsection, one should note the new contribution $\left\langle A^{(S)}\right\rangle$ to the soft breaking $(A-3)$ parameter. This leads to a novel aspect since the magnitude of the soft SUSY breaking parameter in the conventional visible sector, that is involving the $\widetilde{\Phi}$ fields, can now depend on the dynamics of a visible sector field! Moreover, since $V_{\mathrm{LE}}^{\mathrm{NSWS}}$, like $V_{\mathrm{LE}}^{\mathrm{SWS}}$, is supposed to be generated at a high scale where SUSY is broken in the hidden sector, this scale does not need to match to the one where the $S$-fields develop a VEV, the latter being presumably lower. It follows that the running of the conventional soft SUSY breaking parameters can be modified by a different initial condition at the scale where the VEV of the $S$ fields sets in. This is not to be confused with the usual threshold effects due to the decoupling of heavy states in the running quantities, and obviously not with gauge mediated SUSY breaking scenarios-like effects since the mediation is only gravitational. A more drastic scenario obtains if, as mentioned above, the gravitino mass itself is triggered by the VEVs of the $S$-fields. For instance if $\left|M_{10}^{2}\left\langle\omega_{10}(z)\right\rangle\right| \ll\left|M_{11}\left\langle\omega_{11}(z)\right\rangle \sum \mu_{q}^{*}\left\langle S^{q}\right\rangle\right|$ then the bulk of the gravity mediation SUSY breaking to the observable sector occurs not at the scale where SUSY is broken but at the scale where the $S$-fields acquire a VEV. Moreover, in this case $\left\langle A^{(S)}\right\rangle \approx \mathcal{O}(1)$ leading to a sensible modification of $(A-3)$.

The bilinear and linear contributions in the $S$ fields of the last line, Eq. (4.30), are unusual. They are formally not enhanced by powers of $m_{p \ell}$ as expected for the $S$-fields sector. However they can be large since $M \sim \mathcal{O}\left(\left(m_{3 / 2} m_{p \ell}\right)^{1 / 2}\right)$ as seen from Eq. (4.23), so that on general grounds $M$ is related to the SUSY breaking scale $M_{S}=\left(\sqrt{3} m_{3 / 2} m_{p \ell}\right)^{1 / 2}$ up to some numerical factors. They can also play an important role in triggering the magnitudes of $\left\langle S^{p}\right\rangle$. These VEVs are supposed to remain consistently much below $m_{p \ell}$ which is not in general dynamically guaranteed. We come back to this last issue in Section IV C for the simplest NSWS case. We note also in passing that in both SWS and NSWS examples we assumed for simplicity $\langle h\rangle$ to be real-valued, cf. Eqs. $(4.14,4.24)$. In the case where $\langle h\rangle$ develops a phase, there is in the SWS the possibility to choose $\Xi$ in such a way that this phase is not physical and can be absorbed in a redefinition of the fields in the terms proportional to $m_{3 / 2}$ in Eq. (4.15). This contrasts with the NSWS case where the presence of terms linear in $S$ forbids such a redefinition.

Hard breaking terms: The terms containing $A^{(S)}$ in Eqs. $(4.27,4.28,4.29)$ as well as the term containing $A_{i}^{\prime(S)}$ in Eq. (4.28) are not of the soft breaking type due to their dependence on $S^{q *}$ [45]. We will see that they generically lead to hard breaking. The presence of such hard SUSY breaking terms in $V_{\mathrm{LE}}^{\mathrm{NSWS}}$ is an uncommon feature. It is important to identify the underlying reason for the appearance of these terms as it is usually taken for granted that gravity mediated scenarios lead only to soft breaking at low energy. In fact hard breaking terms are present in the standard scenarios and can be traced back to the presence nonrenormalizable operators in the Supergravity potential

\footnotetext{
${ }^{4}$ Here we are only interested in the structure of the SUSY breaking terms. A thourough treatment would have required a detailed study of the vacuum structure and an assessment of an almost vanishing cosmological constant, as well as the consistency requirement $\langle S\rangle \ll \mathcal{O}\left(m_{p \ell}\right)$. This will be carried out in some detail in a simpler setting in Section IV C.
} 
that are suppressed by negative powers of $m_{p \ell} .^{5}$ One can easily see this in the simple setting of Section IVB 1 ; take for example the second term in Eq. (4.11), $\exp \left(\frac{\left|\zeta^{i}\right|^{2}+\left|\Phi^{a}\right|^{2}}{m_{p \ell}^{2}}\right)\left|g_{a}+\frac{\Phi^{a *}}{m_{p \ell}^{2}} W\right|^{2}$. It leads after SUSY breaking to the term $\left(m_{3 / 2} / m_{p \ell}^{2}\right) \Phi^{a *} \Phi^{a} \widehat{g(\Phi)}+h . c$. in the potential, which is clearly not of the soft breaking type[45] even when $g(\Phi)$ contains only renormalizable operators, unless $g$ is a trivial constant, $g(\Phi) \sim m^{3}$ with $m$ some mass scale. Note that the same term is obtained if instead of Eq. (4.10) we choose $W_{2}(z)=0$ and $W_{1}(z)=m_{p \ell}^{-1} h(\zeta)$, since now one negative power of $m_{p \ell}$ is absorbed in the gravitino mass. Other hard breaking terms appear if the hidden sector fields are excited around their VEVs. The leading ones can be read from the soft terms in Eq. (4.15) and Eq. (4.28) by the replacement $b_{i} \rightarrow \zeta^{i} / m_{p \ell}$ in the $A$ parameter. The two types of hard breaking, the one involving only visible sector fields and the one involving visible and hidden sector fields do not appear in Eq. (4.15) for two distinct reasons: the first type is suppressed by two powers of $m_{p \ell}$, and the second type, because hidden sector fields are not excited in the low energy visible sector. The situation is different as concerns the $S$ fields in the NSWS case, because on the one hand they appear in parts of the superpotential where usually only hidden sector fields reside, and on the other hand they are excited at low energy in the visible sector. Whence the appearance of the $A^{(S)}$ and $A_{i}^{\prime(S)}$ terms in $V_{\mathrm{LE}}^{\mathrm{NSWS}}$, that are suppressed by only one negative power of $m_{p \ell}$.

In order to assess whether the terms $A^{(S)}$ and $A_{i}^{\prime(S)}$ indeed induce SUSY breaking we consider now the F-terms VEVs associated with the $S$ fields and hidden sector fields. Assuming no contribution to SUSY breaking from the purely visible sector, one has from Eqs. (2.10, $4.17-4.19)$ :

$$
\begin{aligned}
\left\langle F_{S^{r *}}\right\rangle & \sim m_{p \ell} M_{11}\left\langle\omega_{11}\right\rangle \mu_{r}^{*}+\frac{\left\langle S^{r}\right\rangle^{*}}{m_{p \ell}}\left(M_{10}^{2}\left\langle\omega_{10}\right\rangle+M_{11}\left\langle\omega_{11}\right\rangle \mu_{q}^{*}\left\langle S^{q}\right\rangle\right), \\
\left\langle F_{\zeta^{*}}\right\rangle & \sim M_{10}^{2}\left\langle\frac{\partial \omega_{10}}{\partial z}\right\rangle+M_{11}\left\langle\frac{\partial \omega_{11}}{\partial z}\right\rangle \mu_{q}^{*}\left\langle S^{q}\right\rangle+\langle z\rangle^{*}\left(M_{10}^{2}\left\langle\omega_{10}\right\rangle+M_{11}\left\langle\omega_{11}\right\rangle \mu_{q}^{*}\left\langle S^{q}\right\rangle\right),
\end{aligned}
$$

where the repeated $q$ index indicates a sum and $\zeta, z$ span all the hidden sector fields $\zeta^{i}, z^{i}$. One sees from these expressions that $\left\langle\omega_{11}\right\rangle \neq 0$ contributes to SUSY breaking irrespective of the values of $\left\langle S^{q}\right\rangle$, while $\left\langle\frac{\partial \omega_{11}}{\partial z}\right\rangle \neq 0$ would contribute only if at least one $S^{q}$ develops a nonvanishing VEV. It follows that the $A^{(S)}$ term will always contribute hard breaking of SUSY while the contribution of $A_{i}^{\prime(S)}$ to the hard breaking will depend on the dynamics of the $S$ fields. The magnitude of these hard breaking terms will be discussed further in the following two subsections.

\section{A simple non-Soni-Weldon solution}

We consider now the NSWS in the simple configuration where the $\Xi$ function in Eq. (3.8) does not depend on the $S$-type fields. This configuration is even mandatory in the case of a singleton partition of the $S$-type fields set. In this case all the $\xi(z)$ functions can be taken vanishing since $\Xi$ is $S$-independent. Equation (3.10) becomes trivial and non-constraining, implying that one can choose $P=k_{1}$, and $n_{p}=1, \forall p$ in Eq. (3.11). The superpotential of the NSWS simplifies to,

$$
W(z, S, \widetilde{\Phi})=m_{p \ell}\left[W_{1,0}(z)+S^{p} W_{1, p}(z)\right]+W_{0}(z, \widetilde{\Phi})+S^{p} W_{0, p}(z)
$$

where $W_{1,0}(z), W_{1, p}(z), W_{0, p}(z)$ and $W_{0}(z, \widetilde{\Phi})$ are arbitrary holomorphic functions of $z^{i}$ and $\widetilde{\Phi}^{a}$, whereas $S^{p}$ stands for an arbitrary number $k_{1}$ of $S$-type fields in the visible sector, and we denote by $W_{0}$ the $S$-independent $\Xi$ function. Note also that the $k_{1}$ functions $W_{1, p}(z)$, corresponding to the partition sum appearing in Eq. (3.7), are now all independent functions. The Kähler potential is minimal and given by Eq. (4.17). The simple dependence on $S^{p}$ in Eqs. (4.17, 4.37) will allow to carry out a thorough study of the vacuum structure and to determine conditions on the hidden sector that guarantee $\langle S\rangle \ll m_{p \ell}$. It will also show an important implication on the magnitude of the hard breaking

5 Given that $N=1$ Supergravity breaking can be recast in terms of a global supersymmetric nonrenormalizable theory (see for instance chapter 8 of [46]), it is in principle possible to classify the hard breaking terms using supergraph techniques[47] as was done in [45], by allowing spurion couplings beyond power counting renormalizability criteria. 
terms. Making use of $(4.37),(4.17)$, the potential (2.1) can be recast in the following form:

$$
\begin{aligned}
V(z, S, \widetilde{\Phi})= & \exp \left(z^{i} z^{i *}+\frac{S^{p} S^{p *}+\widetilde{\Phi}^{a} \widetilde{\Phi}^{a *}}{m_{p \ell}^{2}}\right)\left[m_{p \ell}^{2} V_{2}(z)+m_{p \ell} V_{1}(z)+V_{0}(z, S, \widetilde{\Phi})\right. \\
& \left.+\frac{V_{-1}(z, S, \widetilde{\Phi})}{m_{p \ell}}+\frac{V_{-2}(z, S, \widetilde{\Phi})}{m_{p \ell}^{2}}+\frac{V_{-3}(z, S, \widetilde{\Phi})}{m_{p \ell}^{3}}+\frac{V_{-4}(z, S, \widetilde{\Phi})}{m_{p \ell}^{4}}\right]
\end{aligned}
$$

with

$$
\begin{aligned}
& V_{2}(z)=W_{1, p} \bar{W}_{1, p}, \\
& V_{1}(z)=W_{0, p} \bar{W}_{1, p}+W_{1, p} \bar{W}_{0, p}, \\
& V_{0}(z, S, \widetilde{\Phi})=\frac{\partial W_{0}}{\partial \widetilde{\Phi}^{a}} \frac{\partial \bar{W}_{0}}{\partial \widetilde{\Phi}^{a *}}+\left(\left|\widetilde{\rho}_{\widetilde{1}_{i}}\right|^{2}-3\right) \widetilde{W}_{1} \overline{\widetilde{W}}_{1}+W_{0, p} \bar{W}_{0, p} \\
& +S^{p} W_{1, p} \widetilde{\widetilde{W}}_{1}+S^{p *} \widetilde{W}_{1} \bar{W}_{1, p}, \\
& V_{-1}(z, S, \widetilde{\Phi})=\left\{\left(\widetilde{\rho}_{\widetilde{1 i}} \overline{\widetilde{\rho}}_{0 i}-3+\widetilde{\Phi}^{a *} \frac{\partial}{\partial \widetilde{\Phi}^{a *}}\right) \widetilde{W}_{1} \bar{W}_{0}+S^{p} W_{1, p} \bar{W}_{0}\right. \\
& \left.+\left(\left(\widetilde{\rho}_{\widetilde{i}} \overline{\widetilde{\rho}}_{0 p i}-2\right) \widetilde{W}_{1}+S^{q} W_{1, q}\right) S^{p *} \bar{W}_{0, p}\right\}+ \text { h.c. } \\
& V_{-2}(z, S, \widetilde{\Phi})=\left[\left|\widetilde{\rho}_{0 i}\right|^{2}-3+\widetilde{\Phi}^{a} \frac{\partial}{\partial \widetilde{\Phi}^{a}}+\widetilde{\Phi}^{a *} \frac{\partial}{\partial \widetilde{\Phi}^{a *}}\right] W_{0} \bar{W}_{0}+\left(\widetilde{\Phi}^{a} \widetilde{\Phi}^{a *}+S^{p} S^{p *}\right) \widetilde{W}_{1} \overline{\widetilde{W}}_{1} \\
& +S^{p} S^{q *}\left(\widetilde{\rho}_{0 p i} \overline{\widetilde{\rho}}_{0 q i}-1\right) W_{0, p} \bar{W}_{0, q} \\
& +\left\{S^{p}\left[\widetilde{\rho}_{0 p i} \overline{\widetilde{\rho}}_{0 i}-2+\widetilde{\Phi}^{a *} \frac{\partial}{\partial \widetilde{\Phi}^{a *}}\right] W_{0, p} \bar{W}_{0}+\text { h.c. }\right\}, \\
& V_{-3}(z, S, \widetilde{\Phi})=\left(S^{p} S^{p *}+\widetilde{\Phi}^{a} \widetilde{\Phi}^{a *}\right)\left(\bar{W}_{0}+S^{q *} \bar{W}_{0, q}\right) \widetilde{W}_{1}+\text { h.c. }, \\
& V_{-4}(z, S, \widetilde{\Phi})=\left(S^{p} S^{p *}+\widetilde{\Phi}^{a} \widetilde{\Phi}^{a *}\right)\left(W_{0}+S^{q} W_{0, q}\right)\left(\bar{W}_{0}+S^{r *} \bar{W}_{0, r}\right),
\end{aligned}
$$

where we have defined the functions

$$
\begin{aligned}
& \widetilde{\rho}_{\widetilde{1} i}=\frac{\partial}{\partial z^{i}}\left(\frac{K}{m_{p \ell}^{2}}+\ln \frac{\widetilde{W}_{1}}{m_{p \ell}^{2}}\right), \\
& \widetilde{\rho}_{0 i}=\frac{\partial}{\partial z^{i}}\left(\frac{K}{m_{p \ell}^{2}}+\ln \frac{W_{0}}{m_{p \ell}^{3}}\right), \\
& \widetilde{\rho}_{0 p i}=\frac{\partial}{\partial z^{i}}\left(\frac{K}{m_{p \ell}^{2}}+\ln \frac{W_{0, p}}{m_{p \ell}^{2}}\right),
\end{aligned}
$$

with $\widetilde{W}_{1} \equiv W_{1,0}+S^{p} W_{1, p}{ }^{6}$ Apart from the exponential prefactor, the potential is quartic in the $S$-fields. A first general remark is that the couplings of the $S$-fields to the other observable sector fields $\widetilde{\Phi}$ appear only in the terms $V_{-1,-2,-3,-4}$ with negative powers of $m_{p \ell}$ in the potential, while the couplings of the $S$ fields to the (reduced) hidden sector fields $z$ occur already in the $V_{0}$ term. This does not allow to decide in advance on the relative magnitudes of these couplings since the $z$ fields contain a Planck mass suppression, and furthermore different physical scales lighter than $m_{p \ell}$ that are expected to exist, would affect differently the magnitudes of these couplings. To proceed we make explicit these mass scales through the mass dimensions of the various superpotential contributions defining reduced functions and reduced visible sector fields $\phi$ as follows:

\footnotetext{
${ }^{6}$ Unless stated otherwise, summation over repeated indices, including three occurrences of the same index, is understood.
} 


$$
\begin{aligned}
W_{1,0} & =M_{1}^{2} \omega_{1}, \\
W_{1, p} & =M_{2, p} \omega_{1 p},(\text { no sum on } p), \\
W_{0, p} & =M_{3, p}^{2} \omega_{0 p},(\text { no sum on } p), \\
W_{0} & =M_{4}^{3} \omega_{0}(z, \phi), \\
\widetilde{\Phi} & =M_{4} \phi .
\end{aligned}
$$

By definition $M_{1}, M_{2, p}, M_{3, p}, M_{4} \ll m_{p \ell}$, but it is also plausible that the scale $M_{4}$ involving the visible sector is much smaller than the other scales occurring in the hidden sector. As seen on closer inspection of the terms in Eq. (4.38), the potential is, apart from the exponential prefactor, a quartic polynomial in the $S$ fields. The $S$ sector can be thoroughly studied, in particular the structure of the vacuum in the $S$ fields directions. An explicit computation of the VEVs $\left\langle S^{p}\right\rangle$ allows to determine the conditions that guarantee $\left\langle S^{p}\right\rangle \ll m_{p \ell}$, otherwise there would be an inconsistency with the fact that the $S$ fields are not part of the hidden sector! Requiring furthermore the vacuum energy to remain tiny (at the tree-level), the relevant vacuum conditions read:

$$
\begin{aligned}
\langle V\rangle & =0, \\
\left\langle\frac{\partial V}{\partial S^{p *}}\right\rangle & =\left\langle\frac{\partial V}{\partial S^{p}}\right\rangle=0, \\
\left\langle\frac{\partial V}{\partial z^{i *}}\right\rangle & =\left\langle\frac{\partial V}{\partial z^{i}}\right\rangle=0 .
\end{aligned}
$$

In principle one should add to Eqs. $(4.48$ - 4.50) the extrema conditions on the visible sector fields as well. However the latter are expected to hold at much lower scales than the one at which the $S$ fields develop VEVs, and are thus unessential in determining the aforementioned consistency conditions on $\left\langle S^{p}\right\rangle$. Also, necessary positivity conditions on the second order derivatives will be checked a posteriori through the positivity of the squared masses of the $S$ and $z$ fields.

To go further in the study of the vacuum structure, we assume for simplicity hereafter a common scale $M$ in the hidden sector such that

$$
M_{1} \simeq M_{2, p} \simeq M_{3, p}=\xi^{-1} M_{4}=M \equiv \epsilon m_{p \ell},
$$

with $\epsilon \ll 1$ (and possibly $\xi \ll 1$ as well), and consider expansions in powers of the $\epsilon$ parameter. It turns out that keeping only the leading order in $\epsilon$ misses important physical contributions, as the consistancy requirement $\left\langle S^{p}\right\rangle \ll m_{p \ell}$ enforces at this order the potential to be essentially flat in the $S$-fields directions. Including all contributions to $\mathcal{O}\left(\epsilon^{4}\right)$ proves sufficient as it takes into account all the hidden sector and part of the observable sector. ${ }^{7}$

We address now the issue of the size of $\left\langle S^{p}\right\rangle$. For the sake of argument, let us assume from now on that we have one single $S$ field (i.e. $p$ takes only one value), and one single hidden sector field $z$. Parameterizing in general the VEV of $S$ as $\langle S\rangle \equiv e^{i \theta} \lambda m_{p \ell}$, where $\lambda$ is real-valued and positive and $\theta$ is the phase of $S$ at the minimum of the potential, Eq. (4.48) leads to a quartic equation for $\lambda$ with terms controlled by $m_{p \ell}^{2}, M m_{p \ell}$ and $M^{2}$.

This equation can be solved for $\lambda$. However, since $S$ is not in the hidden sector, a consistent solution should correspond to $\lambda=\mathcal{O}\left(\frac{M}{m_{p \ell}}\right) \ll 1$. The requirement $\lambda \ll 1$ cannot be satisfied in general due to dangerous terms in $m_{p \ell}^{2}$ and $M m_{p \ell}$ that do not vanish with vanishing $\lambda$. This puts a necessary constraint on the VEV of $\omega_{1 p}(z)$ in the form,

$$
\left\langle\omega_{1 p}(z)\right\rangle=\epsilon \rho e^{i \alpha} .
$$

Focusing on the case $\langle S\rangle=0$ implies that Eq. (4.48) should be satisfied globally rather than order by order in the $M, m_{p \ell}$ expansion. Equations $(4.48,4.49)$ lead then to the determination of $\rho$,

$$
\begin{aligned}
& \rho_{ \pm}=\left\langle-\operatorname{Re}\left[e^{-i \alpha} \omega_{0 p}(z)\right] \pm\right. \\
& \left.\sqrt{\operatorname{Re}\left[e^{-i \alpha} \omega_{0 p}(z)\right]^{2}+3\left|\omega_{1}(z)\right|^{2}-\left|\omega_{0 p}(z)\right|^{2}-\left|z^{\dagger} \omega_{1}(z)+\omega_{1}^{\prime}(z)\right|^{2}-\xi^{4}\left|\partial_{\phi} \omega_{0}(z, \phi)\right|^{2}}\right\rangle
\end{aligned}
$$

\footnotetext{
7 The neglected higher powers of $\epsilon$, up to power 8, all involve the observable sector.
} 
and to the supplementary constraint,

$$
\left\langle\omega_{1 p}^{\prime}(z)\right\rangle=\left\langle\left(e^{i \alpha} \rho+\omega_{0 p}(z)\right)\left(\frac{2 \bar{\omega}_{1}\left(z^{\dagger}\right)}{z \bar{\omega}_{1}\left(z^{\dagger}\right)+\bar{\omega}_{1}^{\prime}\left(z^{\dagger}\right)}-z^{\dagger}\right)-\omega_{0 p}^{\prime}(z)\right\rangle \epsilon+\mathcal{O}\left(\xi^{3} \epsilon^{2}\right)
$$

where the primes denote derivatives with respect to $z$ (or $\left.z^{\dagger}\right)$. One finds that the potential is bounded from below in the $S$ field direction and the $S$ mass is generically nonvanishing given by:

$$
\begin{aligned}
m_{S}^{2} \equiv & \left\langle\partial_{S^{p *}} \partial_{S^{p}} V\right\rangle_{\mid\langle S\rangle=0}=\frac{e^{\left|b_{i}\right|^{2}} M^{4}}{m_{p \ell}^{2}} \frac{1}{\left\langle\left|z^{\dagger} \omega_{1}(z)+\omega_{1}^{\prime}(z)\right|^{2}\right\rangle} \times \\
& \left\langle\left( 3\left|\omega_{1}(z)\right|^{4}+\left(\left|z^{\dagger} \omega_{1}(z)+\omega_{1}^{\prime}(z)\right|^{2}-3\left|\omega_{1}(z)\right|^{2}\right)^{2}\right.\right. \\
& \left.\left.+\xi^{4}\left(\left|z^{\dagger} \omega_{1}(z)+\omega_{1}^{\prime}(z)\right|^{2}-4\left|\omega_{1}(z)\right|^{2}\right)\left|\partial_{\phi} \omega_{0}(z, \phi)\right|^{2}\right)\right\rangle+\mathcal{O}\left(\frac{M^{5}}{m_{p \ell}^{3}}\right), \\
= & \mathcal{O}\left(m_{3 / 2}^{2}\right),
\end{aligned}
$$

where we neglected the visible sector VEVs as compared to $m_{p \ell}$ and used Eq. (4.23) in the last line. Note that the above expression is the same for both values of $\rho=\rho_{ \pm}$given by Eq. (4.53). Note also that the physical requirement $m_{S}^{2} \geq 0$ is automatically satisfied in the limit $\xi \ll 1$ and would otherwise imply a further constraint on the VEVs of the involved superpotential functions and their derivatives.

Another constraint resulting from Eq. (4.50) with $\langle S\rangle=0$, allows to express $\left\langle\omega_{1}^{\prime \prime}(z)\right\rangle$ in terms of $\left\langle\omega_{1}(z)\right\rangle,\left\langle\omega_{1}^{\prime}(z)\right\rangle,\left\langle\omega_{0}(z, \phi)\right\rangle$ and $\left\langle\partial_{\phi} \omega_{0}(z, \phi)\right\rangle$. However, in contrast with $\left\langle\omega_{1 p}(z)\right\rangle$ and $\left\langle\omega_{1 p}^{\prime}(z)\right\rangle$ as given by Eqs. (4.52, 4.54), we find that $\left\langle\omega_{1}^{\prime \prime}(z)\right\rangle$ is neither $\epsilon$ suppressed nor $\rho_{ \pm}$dependent.

One can also obtain the hidden sector scalar field squared mass which, on the $\langle S\rangle=0$ vacuum, takes the form

$$
m_{\zeta}^{2}=\frac{1}{m_{p \ell}^{2}}\left\langle\partial_{z} \partial_{z^{\dagger}} V\right\rangle_{\mid\langle S\rangle=0},
$$

which reads at leading order in $\epsilon$,

$$
\begin{aligned}
m_{\zeta}^{2} & =\epsilon^{2} m_{p \ell}^{2} e^{\left|b_{i}\right|^{2}}\left\langle\left(\left|\omega_{1 p}(z)\right|^{2}+\left|z^{\dagger} \omega_{1 p}(z)+\omega_{1 p}^{\prime}(z)\right|^{2}\right)\right\rangle, \\
& =\mathcal{O}\left(m_{3 / 2}^{2}\right),
\end{aligned}
$$

where in the last line we took into account the suppression due to Eqs. $(4.52,4.54)$, otherwise $m_{\zeta}^{2}$ would have been $\mathcal{O}\left(M^{2}\right)$. However, this suppression is not generic; masses larger than $m_{3 / 2}$ can be obtained when relaxing the common scale assumption Eq. (4.51) as will be discussed later on. ${ }^{8}$ Although we considered for simplicity a common scale $M$ in the above discussion, it is easy to trace back the dependence on the various $M_{i}$ scales from Eq. (4.47). To clarify the possible physical role the $S^{p}$ fields could play in the NSWS, in particular in the simplest context of a single field studied in this section, a few comments are in order:

$1 /$ the $S^{p}$ are considered as belonging to the visible sector in the sense that, like the $\widetilde{\Phi}^{a}$, they never occur with positive powers of $m_{p \ell}$ in the potential $V$, as one can easily check upon injecting (4.37) in the master form (B1), even though they occur in the first positive power of $m_{p \ell}$ in the superpotential. Thus, like the $\widetilde{\Phi}^{a}$, the $S^{p}$ are low energy degrees of freedom.

$2 / W_{0}\left(z, \widetilde{\Phi}^{a}\right)$ in (4.37) describes the usual matter fields interactions of the supersymmetric SM and its extensions.

$3 /$ the interactions between the $S^{p}$ and the $\widetilde{\Phi}$ fields are always Planck suppressed! This means that although in the visible sector, the $S^{p}$ fields are not 'observable' through SM interactions. There could, however, still be sizable couplings to a beyond the SM observable sector, if the associated scale $M_{4}$ is much larger than the electroweak scale, such as for instance in a GUT sector.

\footnotetext{
${ }^{8}$ Note also that since we are merely interested in orders of magnitude here, we have not considered the contributions of the off-diagonal terms of the mass matrix of the $S, \zeta$ system, as these contribute the same order of magnitude to the eigenmasses.
} 
4/ In the single $S$ field case, the $S$ field is massless before SUSY breaking and acquires a soft mass of order $m_{3 / 2}$ after SUSY breaking.

Given the above properties it is useful to go beyond the common scale assumption Eq. (4.51) by considering hierarchical configurations among the various scales. We discuss hereafter briefly two such configurations (postponing a more elaborate discussion of other configurations to the next subsection):

a)

$$
M_{2, p}, M_{3, p}, M_{4} \ll M_{1} \ll m_{p \ell} .
$$

In this case the leading interactions of the $S$ fields involve the hidden sector fields with couplings scaling as $\left(M_{1} / m_{p \ell}\right)^{4}$.

b)

$$
M_{1}, M_{3, p}, M_{4} \ll M_{2, p} \ll m_{p \ell} .
$$

Here too the leading interactions of the $S$ fields involve the hidden sector fields, however, now the couplings scale as $\left(M_{2, p} / m_{p \ell}\right)^{2}$.

In configuration a) the hidden sector scalar has a mass of $\mathcal{O}\left(M_{1}^{2} / m_{p \ell}\right) \simeq \mathcal{O}\left(m_{3 / 2}^{2}\right)$ and in configuration b) a mass of $\mathcal{O}\left(m_{3 / 2}^{2}\right)$ or $\mathcal{O}\left(M_{4}^{2} / m_{p \ell}\right)$ (if $\left.M_{1}<M_{4}\right)$. If these scales are such that some of the hidden degrees of freedom are excited at the end of an inflationary phase, and thermalized through say some gauge interactions, both a) and b) configurations would feature scattering or production of $S$ field quanta. Depending on the magnitudes of $M_{1}$ and $M_{2, p}$, configuration a) would then possibly provide a setting for freeze-in scenarios for the $S$ fields due to the feeble couplings [48], while configuration b) could rather fit the conventional freeze-out scenario given the larger couplings [49], provided that $S$ is initially thermalized. Note also that in these configurations the decay of the $S$ fields to visible sector fields is highly suppressed, and to the hidden sector fields kinematically forbidden.

The above properties suggest that in specific models, the $S$ can be relatively light, $\mathcal{O}\left(m_{3 / 2}\right)$, and produced in the early universe through the hidden sector interactions at the end of inflation more copiously than by the observable sector degrees of freedom. It will be interesting to investigate further the possibility that the $S$ fields provide a good dark matter candidate. Of course one should consider as well their supersymmetric fermionic partners.

Let us end this subsection by two related comments:

- The hidden sector and the cosmological constant: As stated in the discussion preceding Eq. (4.52) one needs to fine-tune $\left\langle\omega_{1 p}(z)\right\rangle$ to a small number of order $\frac{M}{m_{p \ell}}$ in order to achieve a vanishing vacuum energy. This is somewhat an indication of the criticality of the simple NSWS considered in section IV C, since $\omega_{1 p}(z)$ away from $z=\langle z\rangle$ should definitely be nonzero otherwise the NSWS does not exist altogether. It is worth noting that the issue is expected to be less critical in the case of non-canonical Kähler where, as noted at the end of B 2, there is more freedom in fine-tuning the vacuum energy to a small number by requiring conditions such as Eq.(B108) close to the no-scale condition. We will also see in the next section that relaxing the assumption of common scale $M$ reduces the fine-tuning of $\left\langle\omega_{1 p}(z)\right\rangle$.

- Flat directions and inflation: The model configutation studied in the present section does not feature F-flat directions in the parts of the potential that involve the $S$ field. However, as already noted in Section IIIB, such flat directions become endemic to our general solutions as soon as there are two or more distinct $S$ fields coupled directly, through $\Xi$, to the other $(\tilde{\Phi})$ fields of the visible sector, cf. Eqs. $(3.8,3.9)$. The existence of these directions, together with the fact that they are lifted by the non-vanishing (derivatives of the) $W_{1, p}(z)$ functions in Eq. (3.7), suggest an interesting playground for inflation, since this lifting is expected to be small when the hidden sector scalar fields are close to their VEV values, Eq. (4.54). (see also the discussion in the next section.) Furthermore, since the NSWS favors particle physics models that are specific extensions of the NMSSM, it would be interesting to consider variants to the Higgs-like inflation scenarios $[50,51]$ in this context.

\section{The magnitude of the hard breaking}

It should be clear that the properties of the $S$-field studied in the previous section are not generic to all the NSWS. In particular, the fact that the $S$-field has Planck suppressed couplings not only to the hidden sector but also to the 
visible sector, is a consequence of two assumptions -the superpotential $\Xi$ is assumed to be $S$ independent which is a special case. The mass scales appearing in the hidden sector are either all of the same order and larger than the one in the visible sector, Eq. (4.51) or they follow one of the two other configurations Eq.(4.59) or Eq.(4.60) of the previous section- These assumptions can be relaxed. For instance, with at least two $S$-fields one can easily construct a superpotential $\Xi$ with non suppressed couplings between these fields and the rest of the visible sector, as discussed in Section IV A. There is however a lesson from the previous section that is expected to hold even in the presence of a more general $S$-dependent $\Xi$ : It is always possible to find minima of the potential consistent with the fact that $S$ is not in the hidden sector, i.e. necessarily such that $\langle S\rangle \ll m_{p \ell}$, but at the price of requiring $\left\langle\omega_{1 p}(z)\right\rangle$ and $\left\langle\omega_{1 p}^{\prime}(z)\right\rangle$ to be $\mathcal{O}\left(\frac{M}{m_{p \ell}}\right)$ with $M$ some common physical scale much below $m_{p \ell}$, cf. Eqs. $(4.52,4.54)$. If one assumes a common scale, $M_{10}=M_{11} \equiv M$, in the slightly different NSWS configuration studied in Section IV B 2, the same suppression is expected, i.e. $\left\langle\omega_{11}(z)\right\rangle \approx\left\langle\frac{\partial}{\partial z} \omega_{11}(z)\right\rangle=\mathcal{O}\left(\frac{M}{m_{p \ell}}\right)$, where we assumed for simplicity a single hidden sector field. It then follows from Eqs. $(4.23,4.32,4.33)$ that the hard SUSY breaking terms in Eqs. $(4.28,4.29)$ are $\mathcal{O}\left(\frac{M^{2}}{m_{p \ell}^{2}}\right) \sim \mathcal{O}\left(\frac{m_{3 / 2}}{m_{p \ell}}\right)$, while in Eq. (4.27) the linear term in the $S$ fields is $\mathcal{O}\left(\frac{m_{3 / 2}^{2}}{m_{p \ell}}\right)$ and the quadratic term is $\mathcal{O}\left(\frac{m_{3 / 2}^{2}}{m_{p \ell}^{2}}\right)$. The latter induces at the one-loop level SUSY hard breaking contributions to the diagonal entries of the squared mass matrix of the (conventional) observable sector scalar fields $\tilde{\Phi}^{a}$. These loops of virtual $S^{p}$ (that are quadratically divergent with no cancellations by loops of the $S^{p}$ fermionic partners) are proportional to $m_{S^{p}}^{2}$. However, since $m_{S^{p}}^{2} \sim \mathcal{O}\left(m_{3 / 2}^{2}\right)$, cf. Eq. (4.55), the net effect is $\mathcal{O}\left(\frac{m_{3 / 2}^{2}}{m_{p \ell}^{2}}\right)$, up to loop factors. Furthermore, if the superpotential contains a term similar to the first term of Eq. (4.2), then SUSY hard breaking contributions to the off-diagonal entries of the $H_{u}, H_{d}$ squared mass matrix are similarly generated at the one-loop level proportional to $m_{S^{p}}^{2}$. Since $m_{S^{p}}^{2} \sim \mathcal{O}\left(m_{3 / 2}^{2}\right)$, the net off-diagonal contribution to the squared mass matrix is $\mathcal{O}\left(\frac{m_{3 / 2}}{m_{p \ell}}\right)$. However, despite this formal suppression, a substantial effect could still be obtained by appealing to some model-dependence in the hidden sector such that

$$
\left\langle\left|z^{\dagger} \omega_{1}(z)+\omega_{1}^{\prime}(z)\right|^{2}\right\rangle \ll 1 .
$$

Indeed, as seen from Eq. (4.55), this configuration can in principle allow for parametrically large $m_{S^{p}}$.

The situation can be different if the common scale assumption is relaxed while keeping for simplicity $\left\langle S^{p}\right\rangle \simeq 0$; in this case the discussion is carried out mainly in terms of $M_{1}$ and $M_{4}$. Indeed, the dependence on $M_{2, p}, M_{3, p}$ drops out from the $S, \zeta$ mass matrix once Eq. (4.48) (zero cosmological constant) and Eq. (4.49) are imposed, as well as from the gravitino mass because we take $\left\langle S^{p}\right\rangle \simeq 0$. The gravitino mass scales as

$$
m_{3 / 2} \sim\left|\frac{M_{1}^{2}}{m_{p \ell}}\left\langle\omega_{1}\right\rangle+\frac{M_{4}^{3}}{m_{p \ell}^{2}}\left\langle\omega_{0}\right\rangle\right|,
$$

where we include now the contribution from the visible sector that we had neglected for simplicity in section IV B 2, as stated after Eq. (4.23). (Here $M_{4}$ denotes the largest physical scale in the visible sector and would obviously not contribute to the gravitino mass if it corresponded to the electroweak scale.) It also follows, as a consequence of relaxing the common scale assumption, that $\left\langle\omega_{1 p}(z)\right\rangle$ and $\left\langle\omega_{1 p}^{\prime}(z)\right\rangle$ become less fine-tuned than in Eqs. $(4.52,4.54)$, and are of order $\max \left\{\frac{m_{3 / 2}}{M_{2 p}}, \frac{M_{1}^{2}}{M_{2 p} m_{p \ell}}, \frac{M_{3 p}^{2}}{M_{2 p} m_{p \ell}}\right\}$. Moreover, since $\left\langle\left|\left\langle\omega_{0}\right\rangle\right|\right\rangle$ and $\left\langle\left|\left\langle\omega_{1}\right\rangle\right|\right\rangle$, are expected to be typically $\mathcal{O}(1)$, one identifies three relevant mass scale hierarchy configurations:

$$
\begin{gathered}
\left(\frac{M_{4}^{3}}{m_{p \ell}}\right)^{1 / 2} \ll M_{1} \ll m_{p \ell}, \\
\left(M_{1}^{2} m_{p \ell}\right)^{1 / 3} \ll M_{4} \ll m_{p \ell}, \\
M_{4} \sim \mathcal{O}\left(\left(M_{1}^{2} m_{p \ell}\right)^{1 / 3}\right) \ll m_{p \ell} .
\end{gathered}
$$

The first configuration, Eq. (4.63), is compatible with Eq. (4.59), while configurations $(4.64,4.65)$ can be realized only if $M_{1} \ll M_{4}$. Computing the $S$ and $\zeta$ eigenmasses in this more general case (but assuming for simplicity one $S$ and one $\zeta$ fields), one finds that in configurations (4.63) and (4.64) the $S$ field mass is again $\mathcal{O}\left(m_{3 / 2}\right)$ and the resulting hard breaking contributions to the visible sector squared masses is again $\mathcal{O}\left(\frac{m_{3 / 2}^{2}}{m_{p \ell}^{2}}\right)$ suppressed. In contrast, 
configuration (4.65) allows to decrease significantly $m_{3 / 2}$ with respect to $M_{1}$ if the relative complex phase $\theta$ between $\left\langle\omega_{1}\right\rangle$ and $\left\langle\omega_{0}\right\rangle$ satisfies $\frac{2 \pi}{3} \supsetneqq|\theta| \leq \pi$. The $S$ field mass is now controlled by $M_{1}$ for $M_{1} \gg \sqrt{m_{3 / 2} m_{p \ell}}$, and $m_{S}^{2}$ becomes $\mathcal{O}\left(M_{1}^{2} m_{3 / 2} / m_{p \ell}\right)$. Considering again the SUSY hard breaking contribution from Eq. (4.27), the effect on the squared masses of the scalar visible sector is now $\mathcal{O}\left(M_{1}^{6} /\left(16 \pi^{2} m_{3 / 2} m_{p \ell}^{5}\right)\right)$ (where we have included a $1 / 16 \pi^{2}$ loop factor). As an illustration, for $m_{3 / 2} \sim \mathcal{O}(1) \mathrm{TeV}$ and $M_{1} \sim \mathcal{O}\left(10^{16}\right) \mathrm{GeV}$ this effect can easily lead to a $\sim 20 \%$ increase in the scalar masses. Moreover, as mentioned above, if a term of the form $\lambda \mathcal{U} H_{u} \cdot H_{d}$ is present in the superpotential, cf. Eq. (4.2), the hard SUSY breaking term in Eq. (4.28) would contribute to the off-diagonal squared mass entries in the Higgs sector, which becomes now $\mathcal{O}\left(M_{1}^{4} /\left(16 \pi^{2} m_{3 / 2} m_{p \ell}^{3}\right)\right)$. A sizable effect that increases the squared mass of the lightest Higgs state can ensue. For example, a $\sim 5-20 \%$ loop correction to the tree-level coupling $\lambda$ obtains, for $m_{3 / 2} \sim \mathcal{O}(1) \mathrm{TeV}$, when $M_{1} \lesssim \mathcal{O}\left(10^{15}\right) \mathrm{GeV}$ and $M_{4} \sim \mathcal{O}\left(10^{16}\right) \mathrm{GeV}$. (Recall that $M_{4}$ is the largest scale in the visible sector, which of course does not preclude the presence of much smaller scales, such as the electroweak scale, that would however not play a role in the effects of hard breaking.)

The above features favor the presence of very high scales both in the visible (GUT scale) and hidden sectors. They may be welcome as they provide new sources to increase the SM-like $\mathcal{H}$-boson mass and the other SUSY scalar partners. This is expected to reduce the amount of fine-tuning required to account simultaneously for the $125 \mathrm{GeV}$ mass of the former, the increasing experimental exclusion limits on the masses of the latter, and the $Z$-boson mass through radiative electroweak symmetry breaking in minimal and next-to-minimal SUSY extensions. A detailed phenomenological study of these features lies outside the scope of the present paper. We note, however, that although a sizable hard SUSY breaking is likely to modify the rational about TeV scale naturalness that is usually based on soft SUSY breaking, the hierarchy problem appears in the present scenario embodied in the large cancellation in Eq. (4.62) if one requires electroweak scale gravitino mass. Another option would be to allude to model-dependent configurations in the hidden sector that could lead to Eq. (4.61).

The fermionic $S$-sector: Throughout this paper we have discussed only the scalar components of the $S$-sector. The consistency requirement (2.12) which has been realized through the $F$-term potential of the scalar sector should be automatically satisfied, due to Supersymmetry, in the fermionic sector. (Note that the $D$-term contributions to the potential, Eq. (2.8) always satisfy (2.12) as long as the hidden sector is not charged under visible sector gauge groups.) One can then in principle construct the full Lagrangian involving the $S$-type chiral superfields using the well-known recipes. The fermion-scalar couplings are obtained from the Lagrangian term

$$
-\frac{1}{2} e \exp \left(K / 2 m_{p \ell}^{2}\right) \mathfrak{D}_{I} \mathcal{D}_{J} W \chi^{I} \chi^{J}+\text { h.c. },
$$

where $\mathcal{D}_{J} W$ is as defined in Eq. (2.2),

$\mathfrak{D}_{I} \mathcal{D}_{J} W \equiv W_{I J}+\frac{1}{m_{p \ell}^{2}}\left(K_{I J} W+K_{I} \mathcal{D}_{J} W+K_{J} \mathcal{D}_{I} W-K_{I} K_{J} W\right)-\Gamma_{I J}^{K} \mathcal{D}_{K} W$, the $\Gamma_{I J}^{K}$ being the Christoffel symbols associated with the Kähler manifold, and the $\chi^{I}$ scan the fermionic components of all hidden and visible sectors; see [24] for further details. For instance, from Eqs. (3.1, 3.6 -3.8) one obtains readily the couplings of the NSWS case. We do not discuss further the other parts of the Supegravity Lagrangian in the present paper.

\section{CONCLUSION}

Guided by the familiar consistency requirement of separation between as widely different scales as the Planck and electroweak scales, we have proven in this paper the existence of new possibilities, within $N=1$ Supergravity, for the construction of low energy supersymmetric models consistent with gravity mediation of SUSY breaking to the observable sector in the flat space-time limit. In the minimal Kähler case, and assuming (some of) the hidden sector VEVs to be of the order of the Planck mass, we provided a detailed proof for a complete classification of the physically consistent superpotentials, recovering the conventional forms and exhibiting a large class of new ones as well. The latter are endowed with a new type of fields that, although in the visible sector, feature unusual properties regarding their couplings to the hidden sector and to the conventional observable sector. We then argued that this can open up new model building possibilities beyond the MSSM where the dynamics of this new type of fields, uncharged under the visible sector gauge symmetries, can modify the conventional structure of the soft SUSY breaking parameters and lead as well to unusual hard breaking terms and typically to a much lighter gravitino than in the conventional gravity mediation scenarios. Moreover, these fields can be naturally charged under symmetries of the hidden sector, but also possibly under other secluded or dark gauge sectors, thus in principle allowing to relate SUSY breaking to spontaneous breaking of the corresponding symmetries in these sectors. Alternatively, in the simplest new form for the superpotential the new fields acquire masses of the same order as those of the conventional visible sector but couple extremely weakly to the latter, suggesting dark matter candidates whose relic density would be rather determined by 
the hidden sector interactions. The most general forms feature flat directions in the multiple new fields sectors that involve as well the hidden sector, and could possibly provide a new playground for inflationary model scenarios. Last but not least, we argued that the occurrence of the hard SUSY breaking terms could have interesting spin-offs for particle physics SUSY phenomenology. These results set the stage for further detailed studies.

Finally, we provided in the non-minimal Kähler case two special new solutions satisfying conditions reminiscent of the no-scale one, and suggesting the existence of much richer structures. A systematic treatment of the non-minimal Kähler case is outside the scope of the present paper and will be the subject of a companion paper.

\section{Appendix A: Preliminaries}

This appendix is devoted to the proofs of Propositions that are used in the next appendix. The reader interested in the main proof of the existence of new solutions can skip the details of the present appendix and go directly to B 1.

Proposition A.1 Let $P\left(z^{i}\right), Q\left(z^{i}\right)$ and $R\left(z^{i}\right)$ be three arbitrary multivariate holomorphic functions satisfying the functional identity

$$
\sum_{i=1}^{n}\left(\frac{\partial Q}{\partial z^{i}}+z^{i *} Q\right)\left(\frac{\partial \bar{P}}{\partial z^{i *}}+z^{i} \bar{P}\right)=\alpha Q \bar{P}+\bar{Q} R
$$

with $i$ labeling $n$ complex fields and $\alpha$ an arbitrary complex number. If $P$ is not identically vanishing, then

$$
Q=0, \forall z^{i},
$$

except for the special case $n=1, \alpha=1$ with $R$ nonzero.

To establish the above Proposition, we proceed by reductio ad absurdum proving that $Q \neq 0$ leads to a contradiction. If $Q \neq 0$ in a domain of the $\left(z^{i}\right)^{\prime}$, then dividing by $Q \bar{P}$ both sides of (A1) one gets,

$$
\sum_{i=1}^{n}\left(\frac{\partial \ln Q}{\partial z^{i}}+z^{i *}\right)\left(\frac{\partial \ln \bar{P}}{\partial z^{i *}}+z^{i}\right)=\alpha+\left(\frac{R}{Q}\right)\left(\frac{\bar{Q}}{\bar{P}}\right)
$$

Then, applying on both sides of Eq.(A2) respectively $\frac{\partial^{2}}{\partial z^{a} \partial z^{b *}}, \frac{\partial^{2}}{\partial z^{a} \partial z^{b}}$ and $\frac{\partial^{2}}{\partial z^{a *} \partial z^{b *}}$, where $a, b$ are two arbitrary indices, one finds respectively

$$
\begin{gathered}
\sum_{i=1}^{n} \frac{\partial^{2} \ln Q}{\partial z^{a} \partial z^{i}} \frac{\partial^{2} \ln \bar{P}}{\partial z^{i *} \partial z^{b *}}+\delta^{a b}=\frac{\partial}{\partial z^{a}}\left(\frac{R}{Q}\right) \frac{\partial}{\partial z^{b *}}\left(\frac{\bar{Q}}{\bar{P}}\right) \\
\sum_{i=1}^{n} \frac{\partial^{3} \ln Q}{\partial z^{a} \partial z^{b} \partial z^{i}}\left(\frac{\partial \ln \bar{P}}{\partial z^{i *}}+z^{i}\right)+2 \frac{\partial^{2} \ln Q}{\partial z^{a} \partial z^{b}}=\left(\frac{\bar{Q}}{\bar{P}}\right) \frac{\partial^{2}}{\partial z^{a} \partial z^{b}}\left(\frac{R}{Q}\right)
\end{gathered}
$$

and

$$
\sum_{i=1}^{n} \frac{\partial^{3} \ln \bar{P}}{\partial z^{a *} \partial z^{b *} \partial z^{i *}}\left(\frac{\partial \ln Q}{\partial z^{i}}+z^{i *}\right)+2 \frac{\partial^{2} \ln \bar{P}}{\partial z^{a *} \partial z^{b *}}=\left(\frac{R}{Q}\right) \frac{\partial^{2}}{\partial z^{a *} \partial z^{b *}}\left(\frac{\bar{Q}}{\bar{P}}\right) .
$$

Moreover, we will need the following lemma which we prove first:

Lemma A.2 Let $Q$ and $P$ be nonzero holomorphic functions of the $n$ fields $z^{i}$ satisfying Eq. (A1), then

$$
\frac{\partial}{\partial z^{j}}\left(\frac{Q}{P}\right) \neq 0, \forall j=1, \ldots, n
$$


Proof. If there exists an $a$ such that

$$
\frac{\partial}{\partial z^{a}}\left(\frac{Q}{P}\right)=0
$$

then

$$
Q\left(z^{1}, \ldots, z^{a}, \ldots, z^{n}\right)=\zeta\left(z^{1}, \ldots, z^{a-1}, z^{a+1} \ldots, z^{n}\right) P\left(z^{1}, \ldots, z^{a}, \ldots, z^{n}\right)
$$

where $\zeta$ is an arbitrary (nonzero) function of all the $z^{i}$ s but $z^{a}$. Injecting this form back into Eq. (A3) and taking $a=b$ one finds

$$
\sum_{i=1}^{n} \frac{\partial^{2} \ln P}{\partial z^{a} \partial z^{i}} \frac{\partial^{2} \ln \bar{P}}{\partial z^{i *} \partial z^{a *}}+1=\sum_{i=1}^{n}\left|\frac{\partial^{2} \ln P}{\partial z^{a} \partial z^{i}}\right|^{2}+1=0
$$

which is obviously impossible to satisfy whatever the form of $P$.

We now prove Proposition A.1

Proof. Let us first define the following $n \times n$ symmetric matrices:

$$
\mathbf{Q}^{a b} \equiv i \frac{\partial^{2} \ln Q}{\partial z^{a} \partial z^{b}}, \quad \overline{\mathbf{P}}^{a b} \equiv i \frac{\partial^{2} \ln \bar{P}}{\partial z^{a *} \partial z^{b *}}
$$

Using obvious notations, (A3) can then be rewritten in matrix form as

$$
\mathbf{Q} \overline{\mathbf{P}}=\mathbf{1}-\nabla_{z}(R / Q) \otimes\left[\nabla_{z}(Q / P)\right]^{\dagger}
$$

Taking the determinant one finds

$$
\begin{aligned}
\operatorname{det} \mathbf{Q} \overline{\mathbf{P}} & =\operatorname{det}\left(\mathbf{1}-\boldsymbol{\nabla}_{z}(R / Q) \otimes\left[\boldsymbol{\nabla}_{z}(Q / P)\right]^{\dagger}\right) \\
& =1-\operatorname{Tr}\left(\boldsymbol{\nabla}_{z}(R / Q) \otimes\left[\boldsymbol{\nabla}_{z}(Q / P)\right]^{\dagger}\right),
\end{aligned}
$$

where in the last equation we used the fact that any $n \times n$ matrix that is given by a tensor product of two vectors has its trace as the only nonzero invariant. We are thus led to considering separately the cases

$\operatorname{Tr}\left(\nabla_{z}(R / Q) \otimes\left[\nabla_{z}(Q / P)\right]^{\dagger}\right) \neq 1$ or $=1:$

i)

$$
\operatorname{Tr}\left(\nabla_{z}(R / Q) \otimes\left[\nabla_{z}(Q / P)\right]^{\dagger}\right) \neq 1
$$

This equation is expected to hold generically for a large class of functions $Q / P, R / Q$, since there cannot be a constraint among the independent fields $z^{i}, z^{i *}$. Equation (A12) then implies det $\mathbf{Q} \cdot \overline{\mathbf{P}} \neq 0$ so that both matrices $\mathbf{Q}$ and $\overline{\mathbf{P}}$ are invertible. It follows from (A10) that

$$
\begin{aligned}
\mathbf{Q} & =(\overline{\mathbf{P}})^{-1}-\left(\boldsymbol{\nabla}_{z}(R / Q) \otimes\left[\boldsymbol{\nabla}_{z}(Q / P)\right]^{\dagger}\right)(\overline{\mathbf{P}})^{-1} \\
& \equiv(\overline{\mathbf{P}})^{-1}+i \boldsymbol{\nabla}_{z}(R / Q) \otimes \boldsymbol{\lambda}_{Q}^{\dagger}
\end{aligned}
$$

where we define the vector

$$
\boldsymbol{\lambda}_{Q}^{\dagger} \equiv i\left[\nabla_{z}(Q / P)\right]^{\dagger}(\overline{\mathbf{P}})^{-1}
$$

Applying $\partial / \partial z^{a}$ to (A14), holomorphy leads to,

$$
\frac{\partial}{\partial z^{a}} \mathbf{Q}=i\left(\frac{\partial}{\partial z^{a}} \nabla_{z}(R / Q)\right) \otimes \boldsymbol{\lambda}_{Q}^{\dagger}, \quad \forall z^{a},
$$


and in component form, cf. (A9),

$$
\frac{\partial^{3} \ln Q}{\partial z^{a} \partial z^{b} \partial z^{c}}=\frac{\partial^{2}(R / Q)}{\partial z^{a} \partial z^{b}} \lambda_{Q}^{c *}, \quad \forall a, b, c=1, \ldots, n, \text { and } \forall z^{a}, z^{b}, z^{c} .
$$

The $\lambda_{Q}^{c *}$ are by definition $z^{i}$-independent. Moreover, if there exists at least one set $(a, b)$ such that $\frac{\partial^{2}(R / Q)}{\partial z^{a} \partial z^{b}} \neq 0$ then the above equations become holomorphically separable for all the $\lambda_{Q}^{c *}$ 's, thus implying that the latter must be $z^{i *}$ independent as well. A set $(a, b)$ with $\frac{\partial^{2}(R / Q)}{\partial z^{a} \partial z^{b}} \neq 0$ should indeed exist. [If not, then $\frac{\partial^{2}(R / Q)}{\partial z^{a} \partial z^{b}}=0$ for all $a, b$, which would imply from (A17) that $\frac{\partial^{3} \ln Q}{\partial z^{a} \partial z^{b} \partial z^{c}}=0$ for all $a, b, c$, and finally plugging both in (A4) would lead to $\frac{\partial^{2} \ln Q}{\partial z^{a} \partial z^{b}}=0$ for all $a, b$, that is $\mathbf{Q}=0$, contradicting the fact that this matrix is invertible, cf. (A9) - (A13).] We thus conclude that the vector $\lambda_{Q}^{\dagger}$ must be a $z^{i}, z^{i *}$-independent quantity. It then follows immediately from the holomorphy structure of (A14) that $\overline{\mathbf{P}}$ should be constant as well. Moreover, the constancy of $\boldsymbol{\lambda}_{Q}^{\dagger}$ and $\overline{\mathbf{P}}$ implies through (A15) the constancy of $\left[\nabla_{z}(Q / P)\right]^{\dagger}$. In components this reads, $\frac{\partial^{3} \ln \bar{P}}{\partial z^{a *} \partial z^{b *} \partial z^{c *}}=0$ and $\frac{\partial^{2}}{\partial z^{a *} \partial z^{b *}}\left(\frac{\bar{Q}}{\bar{P}}\right)=0 \forall a, b, c$. Plugging the latter constraints in (A5), one finds $\frac{\partial^{2} \ln \bar{P}}{\partial z^{a *} \partial z^{b *}}=0 \forall a, b$, thus contradicting the fact that $\overline{\mathbf{P}}$ is invertible.

This completes the proof that (A1) admits no solution with a nonzero function $Q$, when (A13) is satisfied.

ii) let us consider now the non-generic case where

$$
\operatorname{Tr}\left(\nabla_{z}(R / Q) \otimes\left[\nabla_{z}(Q / P)\right]^{\dagger}\right)=1
$$

so that det $\mathbf{Q} \overline{\mathbf{P}}=0$, see (A12), and at least one of the $\mathbf{Q}$ or $\mathbf{P}$ matrices is not invertible. Naively speaking, one expects the sufficient and necessary conditions for the above equation to be: $\frac{\partial}{\partial z^{j}}\left(\frac{R}{Q}\right), \frac{\partial}{\partial z^{j *}}\left(\frac{\bar{Q}}{P}\right)$ nonzero constants for some $j$ 's, and possibly $\frac{\partial}{\partial z^{j}}\left(\frac{R}{Q}\right)=0$ and $\frac{\partial}{\partial z^{j *}}\left(\frac{\bar{Q}}{\bar{P}}\right)$ arbitrary functions for some other $j$ 's. [Recall however that $\frac{\partial}{\partial z^{j *}}\left(\frac{\bar{Q}}{\bar{P}}\right)=0$ is forbidden by Lemma A.2.]

The above naive expectation, true for $n=1$, is not the most general solution when $n>1$ ! We note first that there should exist at least one $k$ such that $\frac{\partial}{\partial z^{k}}\left(\frac{R}{Q}\right) \neq 0 \forall z^{k}$, otherwise (A18) cannot be satisfied. Relabelling $k=1$, the general solution for (A18), treated as a linear partial differential equation for $\bar{Q} / \bar{P}$ takes the form [52, 53],

$$
\frac{\bar{Q}}{\bar{P}}=\frac{z^{1 *}}{\frac{\partial}{\partial z^{1}}\left(\frac{R}{Q}\right)}+\mathcal{H}\left[\xi^{1} z^{2 *}-\xi^{2} z^{1 *}, \ldots, \xi^{1} z^{j *}-\xi^{j} z^{1 *}, \ldots, \xi^{1} z^{n *}-\xi^{n} z^{1 *} ; z\right]
$$

with

$$
\xi^{j} \frac{\partial}{\partial z^{1}}\left(\frac{R}{Q}\right)=\xi^{1} \frac{\partial}{\partial z^{j}}\left(\frac{R}{Q}\right)
$$

and $\mathcal{H}[\ldots ; z]$ denotes an arbitrary function of $2 n-1$ entries, where all the $z^{i *}$ appear in the first $n-1$ entries as given by the specified linear combinations of $z^{i *}$ and $z^{1 *}$, and the $z^{i}$ correspond to the last $n$ entries. It follows that, despite the generality of $\mathcal{H}$, the first term linear in $z^{1 *}$ on the right-hand side of (A19) cannot be reabsorbed in $\mathcal{H}$, albeit at the expense of replacing it by another $z^{j *}$ if $\frac{\partial}{\partial z^{j}}(R / Q) \neq 0$. This might suggest that the requirement of antiholomorphy of the sum of the two terms on the right-hand side (to match the anti-holomporphy of $\bar{Q} / \bar{P}$ ) should be satisfied separately for each term, and thus $\frac{\partial}{\partial z^{1}}(R / Q)$ should be a constant function of the $z^{i}$ 's. Although sufficient, it is not obvious that these requirements are necessary. In general the $\xi^{j}$ 's can also be functions of $z$ such that the anti-holomporphy condition results in a new partial differential equation for $\mathcal{H}$ where non-trivial compensations between the two contributions on the right-hand side of (A19) cannot be a priori excluded. Rather than studying such a differential equation on $\mathcal{H}$ it will prove more efficient to write down the general solution for $\ln \bar{P}$ as dictated by Eq. (A2) assuming now that the functions $\frac{\bar{Q}}{\bar{P}}$ and $\frac{R}{Q}$ take the general forms dictated by (A19) and (A20). The 
general solution of Eq. (A2) then reads ${ }^{9}$

$$
\begin{aligned}
\ln \bar{P}= & \int_{\left(z^{1 *}\right)_{i n}}^{z^{1 *}} \frac{d \tau^{*}}{\tau^{*}+\partial_{z^{1}} \ln Q}\left\{\alpha+\frac{R}{Q}[z] \frac{\bar{Q}}{\bar{P}}\left[\tau^{*}, \cdots\right.\right. \\
& \left.\left.\left(\tau^{*}+\partial_{z^{1}} \ln Q\right) r_{i 1}-\partial_{z^{i}} \ln Q, \cdots,\left(\tau^{*}+\partial_{z^{1}} \ln Q\right) r_{n 1}-\partial_{z^{n}} \ln Q\right]\right\} \\
& -\left(z^{1 *}-\left(z^{1 *}\right)_{i n}\right) \sum_{i \geq 1}^{n} r_{i 1} z^{i}+C\left[r_{21}, \cdots, r_{i 1}, \cdots, r_{n 1} ; z\right]
\end{aligned}
$$

where $\left(z^{1 *}\right)_{\text {in }}$ is an arbitrary initial condition reference points, $C$ an arbitrary function of the $r_{i 1}$ 's and the $z$ fields [the occurence of non-indexed $z$ denotes dependence on all $z^{i}$ fields], and we have defined

$$
r_{i 1} \equiv \frac{\partial_{z^{i}} \ln Q+z^{i *}}{\partial_{z^{1}} \ln Q+z^{1 *}}
$$

The requirement of anti-holomorphy of $\ln \bar{P}$ in all $z^{i}$ 's puts strong constraints on the right-hand side of Eq. (A21). Taken at the special point $z^{1 *}=\left(z^{1 *}\right)_{i n}$, the anti-holomorphy of $\ln \bar{P}$ implies that $C\left[r_{21}, \cdots, r_{i 1}, \cdots, r_{n 1} ; z\right]$ should be anti-holomorphic as well in all $z^{i}$ fields including $z^{1}$ since $\left(z^{1 *}\right)_{\text {in }}$ can be chosen arbitrarily. This constraint is readily satisfied since the function $C$ is arbitrary. Then from Eq. (A21) with $z^{1 *} \neq\left(z^{1 *}\right)_{\text {in }}$ follows that the sum of the first two terms on the right-hand side should be separately anti-holomorphic in all $z^{i}$ s. In particular, the derivative of this sum with respect to $z^{1 *}$ should also be anti-holomorphic. One then obtains the compact condition

$$
\begin{array}{r}
\alpha+\frac{R}{Q}\left[z^{1}, \cdots\right] \frac{\bar{Q}}{\bar{P}}\left[z^{1 *}, \cdots, z^{i *}, \cdots, z^{n *}\right]-\sum_{i \geq 1}^{n}\left(z^{i *}+\partial_{z^{i}} \ln Q\right) z^{i} \\
\equiv\left(z^{1 *}+\partial_{z^{1}} \ln Q\right) \mathcal{F}_{1}\left[z^{*}\right]
\end{array}
$$

where we define

$$
\mathcal{F}_{1}\left[z^{\dagger}\right]=\partial_{z^{1 *}} \ln \bar{P}-\partial_{z^{1 *}} C
$$

which is by assumption anti-holomorphic in all $z^{i *}$ s. Note that in Eq. (A23) all dependence on the $z^{i}$ s has dropped out from $\frac{\bar{Q}}{\bar{P}}$ due to the replacement $\tau^{\star}=z^{1 *}$. It is intuitively clear why this equation cannot hold if $n>1$, whatever the poduct $\frac{R}{Q} \times \frac{\bar{Q}}{P}$ of holomorphic and anti-holomorphic functions may be, due to the presence of the term $\sum_{i \geq 1}^{n} z^{i *} z^{i}$. To proceed with the proof, it is convenient to treat separately the multi-field and single-field cases:

- $n \geq 2$ : applying $\partial^{2} / \partial z^{k *^{2}}$ for a given $k \neq 1$ to Eq. (A23), one obtains

$$
\frac{R}{Q}\left[z^{1}, \cdots\right] \frac{\partial^{2}}{\partial z^{k *^{2}}} \frac{\bar{Q}}{\bar{P}}\left[z^{1 *}, \cdots\right]=\left(z^{1 *}+\partial_{z^{1}} \ln Q\right) \frac{\partial^{2}}{\partial z^{k *^{2}}} \mathcal{F}_{1}\left[z^{1 *}, \cdots\right]
$$

9 recall that the general solution to a multi-variable function $f\left(x_{1}, x_{2}, \ldots x_{n}\right)$ satisfying a first order linear partial differential equation of the form

$$
\sum_{i=1}^{n}\left(a_{i} x_{i}+b_{i}\right) \frac{\partial f}{\partial x_{i}}=B\left(x_{1}, x_{2}, \ldots, x_{n}\right),
$$

where all the $a_{i}$ are nonzero constants, is given by

$$
\begin{aligned}
f\left(x_{1}, x_{2}, \ldots\right)= & \frac{1}{a_{1}} \int_{x_{0}}^{x_{1}} \frac{d x}{x+c_{1}} B\left(x, \cdots,\left(\frac{x+c_{1}}{x_{1}+c_{1}}\right)^{a_{i} / a_{1}}\left(x_{i}+c_{i}\right)-c_{i},\right. \\
& \left.\cdots,\left(\frac{x+c_{1}}{x_{1}+c_{1}}\right)^{a_{n} / a_{1}}\left(x_{n}+c_{n}\right)-c_{n}\right) \\
& +C\left(\frac{x_{2}+c_{2}}{\left(x_{1}+c_{1}\right)^{a_{2} / a_{1}}}, \cdots, \frac{x_{i}+c_{i}}{\left(x_{1}+c_{1}\right)^{a_{i} / a_{1}}}, \cdots, \frac{x_{n}+c_{n}}{\left(x_{1}+c_{1}\right)^{a_{n} / a_{1}}}\right),
\end{aligned}
$$

where $C$ is an arbitrary function of $n-1$ variables, $x_{0}$ an arbitrary reference constant and $c_{i} \equiv \frac{b_{i}}{a_{i}}$. 
which by holomorphy implies either

$$
\frac{\partial^{2}}{\partial z^{k *}} \frac{\bar{Q}}{\bar{P}}=0
$$

or

$$
\frac{R}{Q}=\text { constant and } \partial_{z^{1}} \ln Q=\text { constant } .
$$

Indeed if Eq. (A26) is not satisfied for at least one $k(\neq 1)$ then Eq. (A25) takes the form

$$
\frac{f(z)}{z^{1 *}+g(z)}=h\left(z^{\dagger}\right)
$$

which, from $\partial_{z^{k}} h\left(z^{*}\right)=0$ for all $k$ and the fact that $z^{1 *}$ cannot be a function of the $z$ fields, leads to $f$ and $g$ constant in $z$, whence Eq. (A27). Now the latter equation cannot be satisfied since $\frac{R}{Q}=$ constant in all $z$ fields contradicts Eq. (A18). It follows that Eq. (A26) should be satisfied for all $k \neq 1$. Note that having chosen $z^{1 *}$ as a reference field when writing Eq. (A21) entailed only the assumption that $z^{1 *}+\partial_{z^{1}} \ln Q$ is not a vanishing function of $z^{1 *}$. But this is true for any other $z^{k *}+\partial_{z^{k}} \ln Q$. We could thus rewrite Eq. (A21) in terms of any other reference field $z^{k *}$ and permute the roles of the 1 and $k$ indices in the thread of equations (A21 - A26) thus arriving at

$$
\partial_{z^{1 *}}^{2} \frac{\bar{Q}}{\bar{P}}=0
$$

Condition (A26) should thus be satisfied for all $k$. Furthermore, this condition implies from Eq. (A25) that

$$
\partial_{z^{k *}}^{2} \mathcal{F}_{1}\left[z^{1 *}, \cdots\right]=0, \quad \forall k,
$$

since $z^{1 *}+\partial_{z^{1}} \ln Q$ cannot be a vanishing function. The most general allowed forms for $\frac{\bar{Q}}{\bar{P}}$ and $\mathcal{F}_{1}$ are then,

$$
\begin{gathered}
\frac{\bar{Q}}{\bar{P}}\left[z^{\dagger}\right]=\alpha_{0}+\sum_{i \geq 1}^{n} \alpha_{i} z^{i *}+\sum_{j>i \geq 1}^{n} \alpha_{i j} z^{i *} z^{j *} \\
\mathcal{F}_{1}\left[z^{\dagger}\right]=\beta_{0}+\sum_{i \geq 1}^{n} \beta_{i} z^{i *}+\sum_{j>i \geq 1}^{n} \beta_{i j} z^{i *} z^{j *}
\end{gathered}
$$

where the $\alpha$ 's, $\beta$ 's are ( $z$-independent) constants and the double sums hold only for $i \neq j$. One can now plug these forms back into Eq. (A23) and identify the various monomials. One then immediatly sees that monomials of the form $z^{1 *} z^{i *} z^{j *}$ with $i \neq j$ appear only once, on the right-hand side of Eq. (A23), implying

$$
\beta_{i j}=0 \quad \forall i \neq j
$$

Note also that the monomial $\left(z^{1 *}\right)^{2}$ appears only once, thus

$$
\beta_{1}=0
$$

Then collecting monomials of the form $z^{1 *} z^{i *}$ with $i \neq 1$ implies

$$
\alpha_{i 1} \times \frac{R}{Q}[z]-\beta_{i}=0, \quad \forall i \neq 1 .
$$

Note that the only other function of $z$, namely $\partial_{z^{1}} \ln Q$ does not appear in the above equation as a consequence of Eq. (A33). Since $\frac{R}{Q}[z]$ cannot be a constant, as noted previously, it follows that

$$
\alpha_{i 1}=\beta_{i}=0, \quad \forall i \neq 1
$$

Collecting now all the contributions to the monomials $z^{1 *}$ and $z^{i *}$ with $i \neq 1$ one gets respectively

$$
\alpha_{1} \times \frac{R}{Q}[z]-z^{1}-\beta_{0}=0 \text { and } \alpha_{i} \times \frac{R}{Q}[z]-z^{i}=0, \quad \forall i \neq 1,
$$


where we have used Eqs. (A34, A36) and again $\partial_{z^{1}} \ln Q$ does not contribute. Since the above constraints should hold functionally in $z$, then $\alpha_{1}$ and $\alpha_{i}$ cannot be vanishing. It follows that,

$$
\frac{R}{Q}[z]=\frac{z^{1}+\beta_{0}}{\alpha_{1}}=\frac{z^{i}}{\alpha_{i}}, \quad \forall i \neq 1,
$$

which is obviously a contradictory requirement. Thus $n \geq 2$ does not allow for a consistent solution.

- $n=1$ : in this case of single field the arbitrary function $C$ becomes $z^{*}$ independent, cf. Eqs. (A21, A22), and Eq. (A24) becomes $\mathcal{F}_{1}\left[z^{*}\right]=\partial_{z^{1 *}}(\ln P *)$. In this case Eq. (A23) reduces exactly to Eq. (A2) with $n=1$ and we can use directly the latter or its derivatives. Moreover, Eq. (A18) simplifies to

$$
\left(\frac{R}{Q}\right)^{\prime} \times\left(\frac{\bar{Q}}{\bar{P}}\right)^{\prime}=1
$$

where the primes denote here and in the sequel derivatives with respect to either $z^{1}$ or $z^{1 *}$ consistently. The latter equation and holomorphy imply that $\frac{R}{Q}$ and $\frac{\bar{Q}}{P}$ should be strictly linear in $z^{1}$ and $z^{1 *}$ respectively:

$$
\begin{gathered}
\frac{\bar{Q}}{\bar{P}}\left[z^{1 *}\right]=\alpha_{0}+\alpha_{1} z^{1 *} \\
\frac{R}{Q}\left[z^{1}\right]=\gamma_{0}+\gamma_{1} z^{1} .
\end{gathered}
$$

where the $\alpha$ 's and $\gamma^{\prime}$ 's are complex-valued constants with $\alpha_{1}, \gamma_{1}$ necessarily nonzero and satisfying $\alpha_{1} \gamma_{1}=1$. When combined with Eq. (A3) with $n=1$ it also implies,

$$
(\ln Q)^{\prime \prime}(\ln \bar{P})^{\prime \prime}=0
$$

Moreover, one sees from (A40) that $(\ln Q)^{\prime \prime}$ and $(\ln \bar{P})^{\prime \prime}$ cannot be simultaneously vanishing since $\alpha_{1}$ cannot vanish. Choosing without loss of generality $(\ln \bar{P})^{\prime \prime}=0$ and using equations (A40), (A41) in (A4), leads then immediately to $(\ln P)^{\prime}=\alpha_{0} / \alpha_{1}$. Also equation (A40) yields $(\ln Q)^{\prime}=\alpha_{1}^{*} /\left(\alpha_{1}^{*} z^{1}+\alpha_{0}^{*}\right)+\alpha_{0} / \alpha_{1}$. Injecting these expressions in $(\mathrm{A} 2)$ one finds

$$
\left(\frac{\alpha_{1}^{*}}{\alpha_{1}^{*} z^{1}+\alpha_{0}^{*}}+\frac{\alpha_{0}}{\alpha_{1}}+z^{1 *}\right)\left(\frac{\alpha_{0}^{*}}{\alpha_{1}^{*}}+z^{1}\right)=\alpha+\left(\gamma_{1} z^{1}+\gamma_{0}\right)\left(\alpha_{1} z^{1 *}+\alpha_{0}\right)
$$

Close inspection shows that this equation is valid if and only if: $\gamma_{1}=\left(\alpha_{1}\right)^{-1}, \gamma_{0}=\alpha_{0}^{*}\left|\alpha_{1}^{*}\right|^{-2}$, and $\alpha=1$. This identifies the special case referred to at the end of Proposition A.1 showing the existence of a nonzero $Q$ in this special case. We do not write explicitly this solution here since the relevant value of $\alpha$ in our study is $\neq 1$. This ends the proof for the case where (A18) is valid.

We have thus completed the proof of Proposition A.1, namely that when $P$ is not identically vanishing, equation (A1) implies in general the unique solution $Q=0$.

In the next section we will encounter cases where Proposition A.1 applies directly, but also cases that have either more restricted or more general forms than this Proposition. We give here the two corresponding corollaries.

Corollary A.3 Let $P$ and $R$ be two arbitrary holomorphic functions satisfying the following functional identity

$$
\sum_{i=1}^{n}\left|\frac{\partial P}{\partial z^{i}}+z^{i *} P\right|^{2}=\alpha|P|^{2}+\bar{P} R
$$

with $i$ labeling $n$ complex fields and $\alpha$ an arbitrary complex number. Then the only solution is

$$
P=0, \quad \forall z^{i}
$$

Proof. Clearly, Eq. (A44) is a special case of (A1) where $P=Q$. Then $P=0$ follows immediatly from Lemma A.2, since if $P \neq 0$ then Eq. (A6) cannot hold when $P=Q$. 
Corollary A.4 Let $P_{r}\left(z^{i}\right), Q_{l q}\left(z^{i}\right)$ and $R_{r l q}\left(z^{i}\right)$ be three sets of arbitrary multivariate holomorphic functions satisfying the functional identity

$$
\sum_{i=1}^{n}\left(\frac{\partial Q_{l q}}{\partial z^{i}}+z^{i *} Q_{l q}\right)\left(\frac{\partial \bar{P}_{r}}{\partial z^{i *}}+z^{i} \bar{P}_{r}\right)=\alpha Q_{l q} \bar{P}_{r}+\sum_{p=1}^{k} \bar{Q}_{r p} R_{p l q},
$$

with $i$ labeling $n$ complex fields, $r, l, p, q=1, \ldots, k$ and $\alpha$ an arbitrary complex number. If $P_{r}$ is not identically vanishing for at least one $r$, then generically

$$
Q_{l q}=0, \forall z^{i}, l, q .
$$

The proof is mostly a mere generalization of the proof of Proposition A.1 albeit with some technical complications. For the sake of conciseness we refrain from giving this somewhat lengthy proof here and refer the reader to appendix A of the arXiv version 1 (arXiv:1611.10327v1, [54]).

\section{Appendix B: Proof of the existence of new solutions}

\section{Minimal Kähler}

We give here the main steps of the proof that in the case of canonical Kähler potential Eq. (3.1), equations (3.5) and (3.6) of Section III A constitute together the sufficient and necessary general forms of the superpotential that lead to a consistent low energy Lagrangian.

Collecting in (3.2) all coefficients of the same power $m_{p \ell}^{c}$ with $c \geq 0$ one finds for $V_{M, c}\left[z, z^{\dagger}, \Phi, \Phi^{\dagger}\right]$ :

$$
\begin{aligned}
V_{M, c}\left[z, z^{\dagger}, \Phi, \Phi^{\dagger}\right]= & \sum_{n_{-}^{(0)} \leq n \leq n_{+}^{(0)}} \frac{\partial W_{n}}{\partial \Phi^{a}} \frac{\partial \bar{W}_{c-n}}{\partial \Phi^{a *}} \\
& +\sum_{n_{-}^{(2)} \leq n \leq n_{+}^{(2)}}\left(\left(\frac{\partial W_{n}}{\partial z^{i}}+z^{i *} W_{n}\right)\left(\frac{\partial \bar{W}_{c-n+2}}{\partial z^{i *}}+z^{i} \bar{W}_{c-n+2}\right)\right. \\
& \left.+\Phi^{a} \frac{\partial W_{n}}{\partial \Phi^{a}} \bar{W}_{c-n+2}+\Phi^{a *} \frac{\partial \bar{W}_{n}}{\partial \Phi^{a *}} W_{c-n+2}-3 W_{n} \bar{W}_{c-n+2}\right) \\
& +\sum_{n_{-}^{(4)} \leq n \leq n_{+}^{(4)}} W_{n} \bar{W}_{c-n+4} \Phi^{a *} \Phi^{a}
\end{aligned}
$$

where we define

$$
\begin{aligned}
& n_{+}^{(s)}=\min [M, c+s], \\
& n_{-}^{(s)}=\max [0, c-M+s] .
\end{aligned}
$$

Recall that $W_{r}$ denotes the coefficient of $m_{p \ell}^{r}$ in the superpotential expansion Eq. (2.15) with $r$ an integer verifying $0 \leq r \leq M$ by definition. The latter constraint is expressed in Eq. (B1) through the summation bounds $n_{-}^{(s)} \leq n \leq n_{+}^{(s)}$, with $s=0,2,4$, corresponding to the three different sums occurring in each $m_{p \ell}^{c}$ coefficient. We will refer to these three sums as, 0-sum, 2-sum and 4-sum. Note also that the implicit constraints $n_{-}^{(s)} \leq n_{+}^{(s)}$ yield the conditions under which the corresponding $s$-sum contributes to a given power of $m_{p \ell}$. They can be stated as follows:

$$
\text { a given s-sum contributes to a given } m_{p \ell}^{c} \text { term if and only if } c \leq 2 M-s \text {. }
$$

Recall that requiring the fields $\Phi^{a}$ not to belong to the hidden sector, that is not to appear in parts of $V_{F}$ that diverge in the limit $m_{p \ell} \rightarrow \infty$, cf. (2.12), is equivalent to requiring that each $V_{M, c}\left[z, z^{\dagger}, \Phi, \Phi^{\dagger}\right]$ with $c>0$ in Eq. (B1), should be functionally independent of $\Phi, \Phi^{\dagger}$, cf. Eq. (3.4). We write this as

$$
V_{M, c}\left[z, z^{\dagger}, \Phi, \Phi^{\dagger}\right] \sim_{\Phi} 0
$$

and take throughout the proof the notation $E \sim_{X} 0$ to mean that $E$ has no functional dependence on $X$ or $X^{\dagger}$. We now study the above master equation for each value of $M$ and each strictly positive value of $c$. 
Note first that if $M=0$ then $c=0$ and there are obviously no conditions to be imposed,

$$
W(z, \Phi)=W_{0}(z, \Phi)
$$

with $W_{0}(z, \Phi)$ an arbitrary function.

We assume now $M \geq 1$ and study all $c$ values in decreasing order.

- $\mathbf{c}=\mathbf{2 M}$ : relevant when $M>0$, the highest power of $m_{p \ell}$ gets according to property B4 contributions only from the 0 -sum in the master equation (B1). The corresonding constraint reads,

$$
\sum_{a}\left|\frac{\partial W_{M}}{\partial \Phi^{a}}\right|^{2} \sim_{\Phi} 0
$$

Since the lefthand side is a sum of positive numbers there can be no cancellation among the $\Phi^{a}$ dependent terms to get $\mathrm{a} \sim \sim_{\Phi} 0$ result. Thus one must have

$$
\left|\frac{\partial W_{M}}{\partial \Phi^{a}}\right|^{2} \sim_{\Phi} 0
$$

separately for each $\Phi^{a}$. It follows that $\frac{\partial W_{M}}{\partial \Phi^{a}}$ should be a $\Phi^{a}$ independent function for all $\Phi^{a}$. The general form for $W_{M}$ is thus

$$
W_{M}(z, \Phi)=W_{M, 0}(z)+\Phi^{a} W_{M, a}(z)
$$

where $W_{M, 0}(z)$ and $W_{M, a}(z)$ denote arbitrary holomorphic functions of the $z^{i}$ s, and the repeated $a$ index is understood as a sum $(a=1, \ldots, k)$.

- $\mathbf{c}=\mathbf{2 M}-\mathbf{1}$ : again, due to B4 only the 0-sum contributes leading to the constraint

$$
\frac{\partial \bar{W}_{M}}{\partial \Phi^{a *}} \frac{\partial W_{M-1}}{\partial \Phi^{a}}+\text { h.c. } \sim_{\Phi} 0,
$$

with a summation on $a$ understood. Using Eq. (B8) this constraint reads,

$$
\bar{W}_{M, a}\left(z^{\dagger}\right) \frac{\partial W_{M-1}}{\partial \Phi^{a}}+\text { h.c. } \sim_{\Phi} 0
$$

which is obviously equivalent to requiring

$$
\bar{W}_{M, a}\left(z^{\dagger}\right) \frac{\partial W_{M-1}}{\partial \Phi^{a}} \sim_{\Phi} 0
$$

[as can be seen for instance by taking the derivative of Eq. (B10) with respect to $\Phi^{b}$ for any $b$ where the h.c. part drops out in this operation due to holomorphy.] We recast Eq. (B11) in the form

$$
\bar{W}_{M, a}\left(z^{\dagger}\right) \frac{\partial W_{M-1}}{\partial \Phi^{a}}=f\left(z, z^{\dagger}\right),
$$

where $f$ denotes an arbitrary function depending only on the hidden sector fields.

We generically assume $f$ to be a nonzero function. By this we mean that there exists at least one connected domain (not just isolated points) in the $z, z^{\dagger}$ field space where $f$ does not vanish. In such a domain Eq. (B12) can be rewritten as

$$
g_{a}\left(z, z^{\dagger}\right) \frac{\partial W_{M-1}}{\partial \Phi^{a}}=1
$$

with $g_{a}\left(z, z^{\dagger}\right) \equiv \frac{\bar{W}_{M, a}\left(z^{\dagger}\right)}{f\left(z, z^{\dagger}\right)}$. Equation (B13) is a linear partial differential equation for $W_{M-1}$ as a function of the $\Phi^{a}$ 's with coefficients independent of these variables. We have already encountered a similar equation in the previous appendix, Eq. (A18). The most general solution of Eq. (B13) will have a form similar to Eq. (A19). Indeed, in order 
for Eq. (B13) to be satisfied at least one $g_{a}$ function (or equivalently one $\bar{W}_{M, a}$ ) should be nonzero, call it $\bar{W}_{M, 1}$. The general solution for $W_{M-1}$ takes then the form (see also [52, 53]),

$$
W_{M-1}\left(z, \Phi^{a}\right)=\frac{f\left(z, z^{\dagger}\right)}{\bar{W}_{M, 1}\left(z^{\dagger}\right)} \Phi^{1}+\Omega_{1}\left(\mathcal{U}_{\Phi}^{12}, \ldots, \mathcal{U}_{\Phi}^{1 a}, \ldots, \mathcal{U}_{\Phi}^{1 k} ; z, z^{\dagger}\right)
$$

where we have defined

$$
\mathcal{U}_{\Phi}^{1 a}=\xi_{a}^{1} \Phi^{a}-\xi^{a, 1} \Phi^{1}, \text { for } a=2, \ldots, k
$$

with $\xi_{a}^{1}$ and $\xi^{a, 1}$ satisfying

$$
\xi_{a}^{1} \bar{W}_{M, a}\left(z^{\dagger}\right)=\xi^{a, 1} \bar{W}_{M, 1}\left(z^{\dagger}\right),(\text { no summation over } a),
$$

and where $\Omega_{1}$ is an arbitrary function of $k-1$ entries with the dependence on combinations of the $\Phi^{a}$ 's as shown in Eq. (B15), and $2 \ell$ entries in the $z^{i}$ 's and $z^{i *}$ s. (Although we assumed $f$ to be nonzero, in fact the case where $f$ is the zero function holds as a special case, putting simply $f=0$ in the general solution Eq. (B14).) To keep the discussion general, we assume that the two sets of parameters $\xi^{a, 1}$ and $\xi_{a}^{1}$ can be any functions of $z, z^{\dagger}$ satisfying Eq. (B16). Note that we write $\xi_{a}^{1}$ rather than simply $\xi^{1}$ to keep track of the fact that $\Phi^{1}$ has been singled out among all the $\Phi^{a}$ in writing the general solution Eq. (B14) and to stress as well that $\xi_{a}^{1}$ in Eq. (B16) is in general different for different values of the $a$ index. Similarly we write $\xi^{a, 1}$, rather than just $\xi^{a}$, to indicate that if we chose another reference field than $\Phi^{1}$, say $\Phi^{2}$, we do not necessarily have $\xi^{a, 1}=\xi^{a, 2}$. Indeed to keep the discussion generic one should consider that there are more than one nonzero $\bar{W}_{M, a}$ apart from $\bar{W}_{M, 1}$. For instance if $\bar{W}_{M, 2} \neq 0$ then one can chose $\Phi^{2}$ to play the role of $\Phi^{1}$, and a general solution similar to Eq. (B14) reads,

$$
W_{M-1}\left(z, \Phi^{a}\right)=\frac{f\left(z, z^{\dagger}\right)}{\bar{W}_{M, 2}\left(z^{\dagger}\right)} \Phi^{2}+\Omega_{2}\left(\mathcal{U}_{\Phi}^{21}, \ldots, \mathcal{U}_{\Phi}^{2 a}, \ldots, \mathcal{U}_{\Phi}^{2 k} ; z, z^{\dagger}\right)
$$

Here the $\mathcal{U}_{\Phi}^{2 a}$ are defined as in Eq.(B15) with 1 replaced by 2, and as just noted we assume in general $\xi_{a}^{1} \neq \xi_{a}^{2}$ and $\xi^{a, 1} \neq \xi^{a, 2}$. Since $\Omega_{1}$ and $\Omega_{2}$ are arbitrary functions of their respective entries, it is legitimate to ask whether each of them can span all possible solutions, so that we can rely on just one of the two equations (B14) or (B17), or else one would need to combine them to get more general solutions. In the former case (B14) and (B17) should be equivalent, implying:

$$
\begin{aligned}
\Omega_{2}\left(\mathcal{U}_{\Phi}^{21}, \ldots, \mathcal{U}_{\Phi}^{2 a}, \ldots, \mathcal{U}_{\Phi}^{2 k} ; z, z^{\dagger}\right)= & \Omega_{1}\left(\mathcal{U}_{\Phi}^{12}, \ldots, \mathcal{U}_{\Phi}^{1 a}, \ldots, \mathcal{U}_{\Phi}^{1 k} ; z, z^{\dagger}\right) \\
& -\frac{f\left(z, z^{\dagger}\right)}{\xi^{2,1} \bar{W}_{M, 1}\left(z^{\dagger}\right)} \mathcal{U}_{\Phi}^{12}
\end{aligned}
$$

where we used Eq. (B16) with $a=2$ when writing the $f$-dependent term. This means that for each $\Omega_{1}$ there should exist an $\Omega_{2}$ satisfying the above equation. The two functions should then be constrained in two ways:

(i) their respective forms should be such that the entries $\mathcal{U}_{\Phi}^{2 a}$ can be transformed into the entries $\mathcal{U}_{\Phi}^{1 a}$.

(ii) $\Omega_{1}$ and/or $\Omega_{2}$ should have linear contributions in order to account for the linear term on the right-hand side of Eq. (B18).

In fact requirement (i) is trivially satisfied due to the identities

$$
\begin{aligned}
& \mathcal{U}_{\Phi}^{21}=-\frac{\xi_{1}^{2}}{\xi^{2,1}} \mathcal{U}_{\Phi}^{12}=-\frac{\xi^{1,2}}{\xi_{2}^{1}} \mathcal{U}_{\Phi}^{12}, \\
& \mathcal{U}_{\Phi}^{2 a}=\frac{\xi_{a}^{2}}{\xi_{a}^{1}} \mathcal{U}_{\Phi}^{1 a}-\frac{\xi^{a, 2}}{\xi_{2}^{1}} \mathcal{U}_{\Phi}^{12},
\end{aligned}
$$

meaning that any function of the $\mathcal{U}_{\Phi}^{2 a}$ entries is automatically a function of the $\mathcal{U}_{\Phi}^{1 a}$ entries and vice-versa. ${ }^{10}$ In this case, requirement (ii) can be arranged for by simply adding a linear term in $\mathcal{U}_{\Phi}^{1 a}$ or $\mathcal{U}_{\Phi}^{2 a}$. One is thus tempted to

10 To establish (B20) we used the identity $\xi^{2,1} / \xi_{2}^{1}=\xi_{a}^{2} \xi^{a, 1} /\left(\xi_{a}^{1} \xi^{a, 2}\right)$ which follows from Eq. (B16) and its equivalent where 1 is replaced by 2. Also (B19) is a consequence of (B20) with $a=1$ and upon use of the obvious identities $\xi^{1,1}=\xi_{1}^{1}$ and $\mathcal{U}^{11}=0$. 
conclude that either of the two forms, (B14) or (B17), is sufficient to span all possible solutions, as well as any other similar form taking a different $\Phi^{a}$, for which $\bar{W}_{M, a}$ is nonzero, as a reference field. However, as we will see below the requirement of holomorphy of $W_{M-1}\left(z, \Phi^{a}\right)$ leads in some instances to obstructions such that (i) and (ii) become insufficient or their generalisation not trivially satisfied.

We note first that the holomorphy in $z$ of $W_{M-1}\left(z, \Phi^{a}\right)$ requires that the last $\ell$ entries in $\Omega_{1}$ should be left out since they cannot be canceled by a $z^{\dagger}$ dependence in $\xi$. This is easily seen by considering the holomorphy of $W_{M-1}$ in $z$ at the field space point $\Phi^{a}=0, \forall a=1, \ldots, k$, implying the holomorphy of $\Omega_{1}\left(0, \ldots, 0, \ldots ; z, z^{\dagger}\right)$ and thus that the last $\ell$ entries should be absent. Taking this into account one finds that the $\xi_{a}^{1}$ 's with $a \neq 1$ should also be holomorphic in $z$. Indeed, the holomorphy of $W_{M-1}\left(z, \Phi^{a}\right)$ at the field space points defined by $\Phi^{a}=0, \forall a=1, \ldots, b-1, b+1, k$ and $\Phi^{b} \neq 0$ for any given $b \neq 1$, implies the holomorphy of $\Omega_{1}\left(0, \ldots, 0, \xi_{b}^{1} \Phi^{b}, 0, \ldots 0 ; z\right)$ and thus the holomorphy of $\xi_{b}^{1}$, $\forall b \neq 1$. By following the same line of reasoning one finds similarly that $\Omega_{2}$ should not have the last $z^{\dagger}$ entries and that the $\xi_{a}^{2}$ 's should be holomorphic in $z$. This obviously holds as well for any function $\Omega_{r}$ and parameters $\xi_{a}^{r}$ 's, when $\Phi^{r}$ plays the role of the reference field.

In contrast, there is no reason at this point to require the $\xi^{a, 1}, \xi^{a, 2}, \ldots \xi^{a, r}$ to be holomorphic, as cancellations are in principle possible within the entries of each $\Omega$ function or between $\Omega$ and the $f\left(z, z^{\dagger}\right)$ dependent terms present in Eqs. (B14), (B17), etc. However, assuming the $\xi^{a, 1}, \xi^{a, 2}, \ldots \xi^{a, r}$ to be non-holomorphic leads to one of the obstructions alluded to after Eq. (B20). Let us illustrate the case with indices 1,2. The coefficient of $\mathcal{U}_{\Phi}^{12}$ in (B20) and possibly in (B19) becomes non-holomorphic as soon as $\xi^{a, 2}$ is non-holomorphic for a subset of the $a$ indices, since as proven above all the $\xi_{a}^{1}$ 's (including $\xi_{2}^{1}$ ) must be holomorphic. Now to show that $\Omega_{2}$ spans all possible solutions, one uses (B19) and (B20) to express $\Omega_{2}$ as a function of the $\mathcal{U}_{\Phi}^{1 a}$ 's. However this involves redefining $\Omega_{2}$ by reabsorbing the coefficients of the $\mathcal{U}_{\Phi}^{1 a}$ 's and in particular $\xi^{a, 2}$, in the last entries of $\Omega_{2}$ containing the explicit dependence on the $z$ fields. But this is impossible in the present case since we showed that holomorphy enforces the last $\ell$ entries of the $\Omega$ 's not to contain $z^{\dagger}$ while $\xi^{a, 2}$ does by assumption, whence the obstruction. This means that in general $\Omega_{2}$, and similarly $\Omega_{1}$ and $\Omega_{i}$, cannot span all possible solutions for $W_{M-1}\left(z, \Phi^{a}\right)$ if they depended only on the $\mathcal{U}_{\Phi}^{2 a}$ 's, $\mathcal{U}_{\Phi}^{1 a}$ 's and $\mathcal{U}_{\Phi}^{i a}$ 's respectively. To obtain the general solution one should include all the $\mathcal{U}_{\Phi}^{i a}$ 's as explicit entries. The most general solution takes then the form,

$$
W_{M-1}\left(z, \Phi^{a}\right)=\frac{f\left(z, z^{\dagger}\right)}{\bar{W}_{M, 1}\left(z^{\dagger}\right)} \Phi^{1}+\Omega\left(\ldots, \mathcal{U}_{\Phi}^{r a}\left(z, z^{\dagger}\right), \ldots ; z\right),
$$

where $r=1,2, \ldots$ label all the fields $\Phi^{r}$ that can be used as reference fields, i.e. for which $\bar{W}_{M, i}\left(z^{\dagger}\right)$ is nonzero, and

$$
\mathcal{U}_{\Phi}^{r a}\left(z, z^{\dagger}\right)=\xi_{a}^{r}(z) \Phi^{a}-\xi^{a, r}\left(z, z^{\dagger}\right) \Phi^{r}, \text { for } a=1, \ldots, k,
$$

with

$$
\left.\xi_{a}^{r}(z) \bar{W}_{M, a}\left(z^{\dagger}\right)=\xi^{a, r}\left(z, z^{\dagger}\right) \bar{W}_{M, r}\left(z^{\dagger}\right) \text {, (no summation over } a\right) .
$$

The first term on the right-hand side of Eq. (B21) is chosen to be the same as in Eq. (B14). This is not a loss of generality. It can be traded for by any other term of the same form, with 1 replaced by $r$, or even an averaged sum of such terms. The reason is that this linear term does not suffer from the aforementioned holomorphy obstruction since the coefficient of the linear term in $\mathcal{U}_{\Phi}^{12}$ in Eq. (B18) is in general non-holomorphic. One can thus always pull out of $\Omega$ a linear term, where the non-holomorphy is embedded in the $\xi^{a, r}$, to match the choice made in Eq. (B21). Note that there can be subsets of $r$ and $a$ for which $\xi^{a, r}$ is holomorphic in $z$. These subsets can be related to each other through (B19), (B20) without any holomorphy obstruction. Given our notations in Eq. (B21) this leads to redundant entries in $\Omega$, but this is harmless and we stick for simplicity to these notations.

Although the holomorphy of each of the $\xi^{a, r}$ 's and a proper choice of $f\left(z, z^{\dagger}\right)$ would be obviously sufficient to ensure the holomorphy of $W_{M-1}$, the reverse is far from straightforward to prove, as cancellations are in principle possible. We address this question in detail below.

But first a crucial remark: as easily seen from Eqs. (B21 - B23), $\Omega$ becomes a totally arbitrary function of the $\Phi^{a}$ 's for the $a$ labels that correspond to vanishing functions $\bar{W}_{M, a}\left(z^{\dagger}\right)$, as this implies $\xi^{a, r}=0$ and $\mathcal{U}_{\Phi}^{r a}=\xi_{a}^{r} \Phi^{a}$, while it remains arbitrary, albeit a function of the field combinations given by $\mathcal{U}_{\Phi}^{r a}$, for the $a$ labels that correspond to nonzero functions $\bar{W}_{M, a}\left(z^{\dagger}\right)$. This mathematical difference leads to an important physical difference which is at the heart of the existence of the NSWS discussed in Section III A. It is thus convenient for the ongoing discussion to label distinctly the $\Phi^{a}$ fields corresponding to the two classes of dependence. We denote by $\widetilde{\Phi}^{a}$ with $a=1, \ldots, k_{0}$ the $k_{0}$ fields that correspond to $\bar{W}_{M, a}\left(z^{\dagger}\right)=0$ and denote by $S^{p}$ with $p=1, \ldots, k_{1}$ the $k_{1}$ fields $\Phi^{r}$ that correspond to $\bar{W}_{M, r}\left(z^{\dagger}\right) \neq 0$, with $k=k_{0}+k_{1}$. We will refer to these two distinct classes as $\widetilde{\Phi}$-type and $S$-type respectively. In terms of this relabeling Eq. (B21) takes the form

$$
W_{M-1}(z, S, \widetilde{\Phi})=\frac{f\left(z, z^{\dagger}\right)}{\bar{W}_{M, 1}\left(z^{\dagger}\right)} S^{1}+\Omega\left(\ldots, \mathcal{U}_{S}^{r p}, \ldots, ; \widetilde{\Phi}^{1}, \ldots, \widetilde{\Phi}^{k_{0}} ; z\right),
$$


where now

$$
\mathcal{U}_{S}^{r p}\left(z, z^{\dagger}\right)=\xi_{p}^{r}(z) S^{p}-\xi^{p, r}\left(z, z^{\dagger}\right) S^{r}, \text { with } r, p=1, \ldots, k_{1}
$$

Note that when there is only one single $S$-type field, say $S^{1}$, then $\mathcal{U}_{S}^{1 p}=0$ and $W_{M-1}$ becomes a sum of a linear function in $S^{1}$ and an arbitrary $S$-independent function of $\widetilde{\Phi}^{a}$ and $z$. Also the case with no $S$-type fields is implicitly included in Eq. (B24) by dropping all dependence on $S$.

We come now to the requirement of the holomorphy of $W_{M-1}$ in $z^{i}$. This is expressed as,

$$
\frac{\partial}{\partial z^{i *}} W_{M-1}(z, S, \widetilde{\Phi})=0, \forall i
$$

The full exploitation of this constraint turned out to be technically very involved. We present it here in the simplified case where $\Omega$ is a function of a single set of $\mathcal{U}_{S}^{r p}$ variables, say $\mathcal{U}_{S}^{1 p}$ with $p=2, \ldots, k_{1}$. In fact this simplified assumption will prove sufficient to obtain the most general solution. Upon use of (B24) equation (B26) implies

$$
\begin{array}{r}
\frac{\partial}{\partial z^{i *}} \frac{f\left(z, z^{\dagger}\right)}{\bar{W}_{M, 1}\left(z^{\dagger}\right)}-\sum_{p \geq 2}^{k_{1}} \frac{\partial \xi^{p}}{\partial z^{i *}} \frac{\partial}{\partial \mathcal{U}_{S}^{1 p}} \Omega\left(\mathcal{U}_{S}^{12}, \ldots, \mathcal{U}_{S}^{1 p}, \ldots, \mathcal{U}_{S}^{1 k_{1}} ; \widetilde{\Phi}^{1}, \ldots, \widetilde{\Phi}^{k_{0}} ; z\right)=0, \\
\forall i=1, \ldots, \ell
\end{array}
$$

where we have used the fact that $\xi_{p}^{1}$ is holomorphic in $z$, denoted the $\xi^{p, 1}\left(z, z^{\dagger}\right)$ by $\xi^{p}$ for simplicity, and dropped out an overall common factor $S^{1}$ when writing the above equation. This equation can be again treated as a linear partial differential equation for $\Omega$ for each given $i$. If $\frac{\partial}{\partial z^{i *}} \frac{f\left(z, z^{\dagger}\right)}{\bar{W}_{M, 1}\left(z^{\dagger}\right)} \neq 0$ there should exist at least one $p \geq 2$, call it $p=2_{b}$, such that $\frac{\partial \xi^{2} b}{\partial z^{i *}} \neq 0$ in order for Eq. (B27) to be satisfied. The general solution of Eq. (B27) for the unknown function $\Omega$ is similar to that of Eq.(B12) for $W_{M-1}$, the $\mathcal{U}_{S}^{1 p}$ playing the role of the $\Phi^{a}$, the $\frac{\partial \xi^{p}}{\partial z^{i *}}$ the role of the $\bar{W}_{M, a}$ of Eqs.(B14, B16), $\frac{\partial}{\partial z^{i *}} \frac{f\left(z, z^{\dagger}\right)}{\bar{W}_{M, 1}\left(z^{\dagger}\right)}$ the role of $f\left(z, z^{\dagger}\right)$ and the index $2_{b}$ the role of the index 1 . It reads,

$$
\Omega=\left(\frac{\partial \xi^{2_{b}}}{\partial z^{i *}}\right)^{-1} \frac{\partial}{\partial z^{i *}} \frac{f\left(z, z^{\dagger}\right)}{\bar{W}_{M, 1}\left(z^{\dagger}\right)} \mathcal{U}_{S}^{12_{b}}+\Xi_{i}\left(\mathcal{V}_{S, i}^{2_{b}}, \ldots, \mathcal{V}_{S, i}^{2_{b} p}, \ldots, \mathcal{V}_{S, i}^{2_{b} k_{1}} ; \ldots, \widetilde{\Phi}^{a} ; z\right)
$$

compare with Eq. (B14), where $\Xi_{i}$ is an arbitrary function of its entries and we have defined

$$
\mathcal{V}_{S, i}^{2_{b} p} \equiv \gamma_{i}^{2_{b}} \mathcal{U}_{S}^{1 p}-\gamma_{i}^{p} \mathcal{U}_{S}^{12_{b}}
$$

(compare with Eq. (B25)), with the $\gamma^{\prime}$ s satisfying

$$
\gamma_{i}^{p} \frac{\partial \xi^{2_{b}}}{\partial z^{i *}}=\gamma_{i}^{2_{b}} \frac{\partial \xi^{p}}{\partial z^{i *}}, \quad \forall p=2, \ldots, k_{1}
$$

(compare with Eq. (B16); note that $\mathcal{V}_{S, i}^{2_{b} 2_{b}}=0$.) The $\Xi_{i}$ functions do not have explicit dependence on $z^{\dagger}$ since $\Omega$ does not. This is a direct consequence of the holomorphy of $W_{M-1}$ in $z$ at the field space point $\widetilde{\Phi}^{a}=S^{p}=0, \forall a, p$. Similarly we note also that $\gamma_{i}^{2 b}$ should be holomorphic in $z$ as a consequence of the holomorphy of $W_{M-1}$ at the field space point $\widetilde{\Phi}^{a}=S^{p}=0, \forall a, p=1, \ldots, r-1, r+1, \ldots$ and $S^{r} \neq 0$ where $r \neq 1,2_{b}$. Indeed the only term left in $W_{M-1}$ in this case, cf. Eqs. (B24, B28), is $\Xi_{i}\left(0, \ldots, 0, \gamma_{i}^{2_{b}} \xi_{r}^{1} S^{r}, 0, \ldots 0 ; z\right)$. The holomorphy of $\gamma_{i}^{2_{b}}$ in $z$ then follows from the holomorphy of $\xi_{r}^{1}$, and of that of $W_{M-1}$ in $z$ at this field space point.

For the sake of generality we have assumed that there is at least one nonholomorphic function $\xi^{2_{b}}$, i.e. $\frac{\partial \xi^{2} b}{\partial z^{i *}} \neq 0$, but we allow as well a subset $\xi^{q}$, with $2_{b}<q_{0 b} \leq q \leq k_{1}$ of the $\xi$ functions to be holomorphic. It follows from Eq. (B30) that $\gamma_{i}^{q}=0$ for this subset, and the associated entries $\mathcal{V}_{S, i}^{2_{b} q}$ reduce to $\mathcal{U}_{S}^{1 q}$ where the common $\gamma_{i}^{2_{b}}$ factors are absorbed in a re-definition of $\Xi_{i}$. Equation (B28) takes then the form

$$
\begin{array}{r}
\Omega=\left(\frac{\partial \xi^{2_{b}}}{\partial z^{i *}}\right)^{-1} \frac{\partial}{\partial z^{i *}} \frac{f\left(z, z^{\dagger}\right)}{\bar{W}_{M, 1}\left(z^{\dagger}\right)} \mathcal{U}_{S}^{12_{b}}+\Xi_{i}\left(\mathcal{V}_{S, i}^{2_{b} 2}, \ldots, \mathcal{V}_{S, i}^{2_{b}\left(q_{0 b}-1\right)},\right. \\
\left.\mathcal{U}_{S}^{1 q_{0 b}} \ldots, \mathcal{U}_{S}^{1 k_{1}} ; \ldots, \widetilde{\Phi}^{a} ; z\right) .
\end{array}
$$

In the following it will prove necessary to distinguish the two generic cases: 
single nonholomorphy: $\frac{\partial}{\partial z^{i *}} \frac{f\left(z, z^{\dagger}\right)}{\bar{W}_{M, 1}\left(z^{\dagger}\right)} \neq 0$ only for one given $i$,

multiple nonholomorphy: $\frac{\partial}{\partial z^{i *}} \frac{f\left(z, z^{\dagger}\right)}{\bar{W}_{M, 1}\left(z^{\dagger}\right)} \neq 0$ for several $i$ 's.

[The special case where $\frac{\partial}{\partial z^{2 *}} \frac{f\left(z, z^{\dagger}\right)}{\bar{W}_{M, 1}\left(z^{\dagger}\right)}=0$ for all $i$ will be addressed later on.] For both single and mutiple nonholomorphies there should exist at least one $p \geq 2$, call it $p=2$, such that $\frac{\partial \xi^{2} b}{\partial z^{i *}} \neq 0$ in order for Eq. (B27) to be satisfied. In both cases there can exist other indices $2_{c}$ of the same type, i.e. satisfying $\frac{\partial \xi^{2} c}{\partial z^{i *}} \neq 0$; moreover, in the multiple nonholomorphy case one should also consider indices satisfying simultaneously $\frac{\partial \xi^{2} d}{\partial z^{i *}} \neq 0$ and $\frac{\partial \xi^{2} d}{\partial z^{j *}} \neq 0$, with $i \neq j$ and $d$ being possibly equal or not to $b$ or $c, \ldots$ These distinctions actually lead to different requirements on the allowed general forms for $W_{M-1}$.

In the single nonholomorphy case this form of $\Omega$ is necessary and sufficient to insure the holomorphy of $W_{M-1}$ and the latter is finally given by,

$$
\begin{aligned}
W_{M-1}(z, S, \widetilde{\Phi})= & \frac{f\left(z, z^{\dagger}\right)}{\bar{W}_{M, 1}\left(z^{\dagger}\right)} S^{1}+\left(\frac{\partial \xi^{2 b}}{\partial z^{i *}}\right)^{-1} \frac{\partial}{\partial z^{i *}} \frac{f\left(z, z^{\dagger}\right)}{\bar{W}_{M, 1}\left(z^{\dagger}\right)} \mathcal{U}_{S}^{12_{b}}+ \\
& \Xi_{i}\left(\mathcal{V}_{S, i}^{2_{b} 2}, \ldots, \mathcal{V}_{S, i}^{2_{b}\left(q_{0 b}-1\right)}, \mathcal{U}_{S}^{1 q_{0 b}} \ldots, \mathcal{U}_{S}^{1 k_{1}} ; \ldots, \widetilde{\Phi}^{a} ; z\right)
\end{aligned}
$$

Note that due to the single nonholomorphy corresponding to one specific index $i$, Eq. (B27) is by definition trivially satisfied for all hidden sector indices other than $i$. Moreover, if there exists at least one other index of the $2_{b}$ type, say $2_{c}$, one can readily recast Eq. (B32) in terms of $2_{c}$ similarly to the discussion following Eq. (B18), due to the identities

$$
\begin{aligned}
& \mathcal{V}_{S, i}^{2_{b} 2_{c}}=-\mathcal{V}_{S, i}^{2_{c} 2_{b}}, \\
& \mathcal{V}_{S, i}^{2_{c} p}=\frac{\gamma^{2 c}}{\gamma^{2_{b}}} \mathcal{V}_{S, i}^{2_{b} p}-\frac{\gamma^{p}}{\gamma^{2 b}} \mathcal{V}_{S, i}^{2_{b} 2_{c}} .
\end{aligned}
$$

In the multiple nonholomorphy case the issue becomes more involved. For instance considering the nonholomorphy with respect to two distinct fiels $z^{i}$ and $z^{j}, \Omega$ can now be written in two different forms. The requirement that they should match brings extra constraints. Consider two indices $2_{b}$ and $2_{c}$ satisfying $\frac{\partial \xi^{2} b}{\partial z^{i *}} \neq 0$ and $\frac{\partial \xi^{2} c}{\partial z^{j *}} \neq 0$. Writing Eq. (B31) for the two sets $\left(2_{b}, i\right)$ and $\left(2_{c}, j\right)$ leads to the requirement,

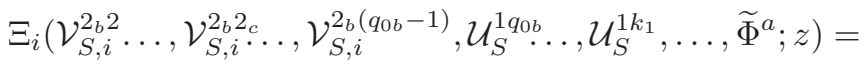

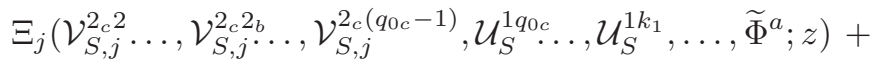

$$
\begin{aligned}
& \left(\frac{\partial \xi^{2 c}}{\partial z^{j *}}\right)^{-1} \frac{\partial}{\partial z^{j *}} \frac{f\left(z, z^{\dagger}\right)}{\bar{W}_{M, 1}\left(z^{\dagger}\right)} \mathcal{U}_{S}^{12_{c}}-\left(\frac{\partial \xi^{2 b}}{\partial z^{i *}}\right)^{-1} \frac{\partial}{\partial z^{i *}} \frac{f\left(z, z^{\dagger}\right)}{\bar{W}_{M, 1}\left(z^{\dagger}\right)} \mathcal{U}_{S}^{12_{b}}
\end{aligned}
$$

for given $i \neq j$. This equation is similar to Eq. (B18) where now the $\Xi$ 's, $\mathcal{V}_{S}$ 's, $\mathcal{U}_{S}$ 's and $2_{b}, 2_{c}$ play respectively the role of the $\Omega$ 's, $\mathcal{U}_{\Phi}$ 's, $\Phi$ 's and 1,2 of that equation. We should stress however that contrary to Eq. (B18) which is allowed not to hold when $\Omega_{1}$ and $\Omega_{2}$ span different sets of solutions, here the constraint (B35) is mandatory since the same function $\Omega$ should satisfy Eq. (B27) for all $i$. One has thus to deal correspondingly with requirements similar to those of (i) and (ii) (see the discussion following Eq.(B18)):

(i)' $\Xi_{i}$ and $\Xi_{j}$ should be such that the entries $\mathcal{V}_{S, i}^{2_{b} p}$ can be transformed into the entries $\mathcal{V}_{S, j}^{2_{c} p}$.

(ii)' $\Xi_{i}$ and/or $\Xi_{j}$ should have linear contributions in order to account for the linear terms in $\mathcal{U}_{S}$ on the right-hand side of Eq. (B35).

Contrary to the case of single nonholomorphy here we do not have identities similar to Eqs. (B33, B34), and (i)', (ii)' are not trivially satisfied. For one thing the linear terms on the right-hand side of Eq. (B35) cannot be recast as a linear term in $\mathcal{V}_{S, i}^{22_{c} 2_{c}}$ or $\mathcal{V}_{S, j}^{22_{b}}$ without assuming additional constraints involving $f\left(z, z^{\dagger}\right)$. For another, unlike Eq. (B33), one cannot relate $\mathcal{V}_{S, i}^{2_{b} 2_{c}}$ to $-\mathcal{V}_{S, j}^{2_{c} 2_{b}}$ as can be seen from Eq. (B29) even with the rescaling freedom in the

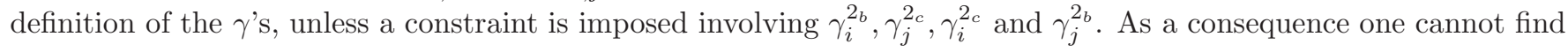


a relation similar to Eq. (B20) that would express the $\mathcal{V}_{S, j}^{2_{c} a}$ 's as functions of the $\mathcal{V}_{S, i}^{2_{b} a}$ 's unless further constraints are imposed on the $\gamma$ 's. We come back later on to the constraints required by (i)' and (ii)'.

To be more specific, we assume without loss of generality that $q_{0 b} \geq q_{0 c}$ so that $\Xi_{j}$ has more $\mathcal{U}_{S}$ entries and less $\mathcal{V}_{S}$ entries than $\Xi_{i}$. As can be seen from the definitions (B25, B29), a $\mathcal{U}_{S}^{1 q}$ entry of $\Xi_{i}$ cannot be obtained from linear combinations involving $\mathcal{U}_{S}$ and $\mathcal{V}_{S}$ entries of $\Xi_{j}$. It can thus only correspond to the same entry $\mathcal{U}_{S}^{1 q}$ of $\Xi_{j}$. It follows that $\Xi_{j}$ should be chosen constant in the extraneous $\mathcal{U}_{S}^{1 q}$ entries that do not appear in $\Xi_{i}$. Similarly one sees from Eqs. (B25, B29) that each $\mathcal{V}_{S, i}^{2_{b} p}$ variable of $\Xi_{i}$ can, at best, be obtained only from a linear combination of the corresponding variable $\mathcal{V}_{S, j}^{2_{c} p}$ and $\mathcal{V}_{S, j}^{2_{c} 2_{b}}$ in $\Xi_{j}$. It follows that $\Xi_{i}$ should also be taken as constant in the extraneous variables $\mathcal{V}_{S, i}^{2_{b} p}$ for which the corresponding variables $\mathcal{V}_{S, j}^{2_{c} p}$ do not appear in $\Xi_{i}$.

For the remaining entries of $\Xi_{i}$ and $\Xi_{j}$, the consistency of Eq. (B35) requires the existence of functions $\alpha, \beta, \kappa, \alpha_{p}$ and $\kappa_{p}$ holomorphic in $z$, such that the following linear combinations are satisfied:

$$
\begin{gathered}
\sum_{p>1} \kappa_{p} \mathcal{V}_{S, i}^{2_{b} p}+\left(\frac{\partial \xi^{2 b}}{\partial z^{i *}}\right)^{-1} \frac{\partial}{\partial z^{i *}} \frac{f\left(z, z^{\dagger}\right)}{\bar{W}_{M, 1}\left(z^{\dagger}\right)} \mathcal{U}_{S}^{12_{b}}= \\
\sum_{p>1} \alpha_{p} \mathcal{V}_{S, j}^{2_{c} p}+\left(\frac{\partial \xi^{22_{c}}}{\partial z^{j *}}\right)^{-1} \frac{\partial}{\partial z^{j *}} \frac{f\left(z, z^{\dagger}\right)}{\bar{W}_{M, 1}\left(z^{\dagger}\right)} \mathcal{U}_{S}^{12_{c}}
\end{gathered}
$$

and

$$
\mathcal{V}_{S, i}^{2_{b} p}+\kappa \mathcal{V}_{S, i}^{2_{b} 2_{c}}=\alpha \mathcal{V}_{S, j}^{2_{c} p}+\beta \mathcal{V}_{S, j}^{2_{c} 2_{b}}, \quad \forall p=2, \cdots, q_{0 b}-1
$$

The holomorphy of $\alpha, \beta, \kappa, \alpha_{p}$ and $\kappa_{p}$ is a necessary requirement. It can be proved analogously to the case of $\gamma_{i}^{2 b}$ discussed after Eq. (B31).

Equation (B36) expresses in the most general way requirement (ii)'. [Note, though, that we assumed without loss of generality $\frac{\partial \xi^{2 c}}{\partial z^{i *}} \neq 0$ and $\frac{\partial \xi^{2} b}{\partial z^{j *}} \neq 0$, so as not to have $\mathcal{U}_{S}^{12_{c}}$ and $\mathcal{U}_{S}^{12_{b}}$ entries respectively in $\Xi_{i}$ and $\Xi_{j}$ that would lead to a special form replacing Eq. (B37).] Equating the coefficients of the independent fields in Eq. (B36), one finds

$$
f\left(z, z^{\dagger}\right)=\sum_{p \geq 1} \bar{W}_{M, p}\left(z^{\dagger}\right) W_{M-1, p}(z)
$$

where we defined

$$
W_{M-1, p}(z) \equiv \xi_{p}^{1} \gamma_{j}^{2_{c}} \alpha_{p}, \forall p \neq 1
$$

and $W_{M-1,1}(z)$ an arbitrary holomorphic function. (For details on the intermediate steps see appendix B.1 of the arXiv version 1 arXiv:1611.10327v1, [54].) Thus $f\left(z, z^{\dagger}\right)$ has exactly the functional form that would have been expected from Eq.(B12).

Equation (B37) expresses requirement (i)', but only in the case where the $\Xi_{i}$ 's have further nonlinear dependences on the $\mathcal{V}_{S}$ entires. Indeed if the $\Xi_{i}$ 's were linear then Eq. (B36) would satisfy by itself both requirements (i)' and (ii)', and Eq. (B37) would be irrelevant. Barring this simple case, general consistency requirements that arise when equating the coefficients of the independent fields $\mathcal{U}_{S}^{12_{b}}, \mathcal{U}_{S}^{12_{c}}$ and $\mathcal{U}_{S}^{1 p}$, in Eq. (B37), cf. Eqs. (B25, B29), lead to the following proportionality relations:

$$
\xi^{p}\left(\xi^{2_{b}}\left(z, z^{\dagger}\right), z\right)=\nu_{p}(z) \xi^{2 b}\left(z, z^{\dagger}\right)
$$

with $\nu_{p}(z)$ an arbitrary holomorphic function in $z$,

$$
\frac{\bar{W}_{M, p}\left(z^{\dagger}\right)}{\bar{W}_{M, 2_{b}}\left(z^{\dagger}\right)}=\frac{\xi_{2_{b}}^{1}}{\xi_{p}^{1}} \frac{\xi^{p}\left(\xi^{2_{b}}\left(z, z^{\dagger}\right), z\right)}{\xi^{2_{b}}\left(z, z^{\dagger}\right)}=\frac{\xi_{2_{b}}^{1}}{\xi_{p}^{1}} \nu_{p}(z) \equiv \mu_{p},
$$

with $\mu$ a constant in $z, z^{\dagger}$, and

$$
\frac{\gamma_{i}^{p}}{\gamma_{i}^{2_{b}}}=\frac{\gamma_{j}^{p}}{\gamma_{j}^{2_{b}}}=\nu_{p}(z)
$$

The above relations result from a rather involved proof that we do not reproduce here. The reader is referred to appendix B.1 of the arXiv version 1 (arXiv:1611.103271v1, [54]) for full details. 
It follows from Eq. (B42) that all the $\gamma_{i}^{p}, \gamma_{j}^{p}$ are holomorphic in $z$ since it was shown that $\gamma_{i}^{2 b}$ and $\gamma_{j}^{2 b}$ should be holomorphic. In contrast $\xi^{2}$ and $\xi^{p}$ (with $p \neq 1$ ) are not forced to be holomorphic. However, one has from Eqs. (B41, B42) that

$$
\gamma_{i}^{p} \xi^{2_{b}}-\gamma_{i}^{2_{b}} \xi^{p}=0
$$

which implies

$$
\begin{aligned}
\mathcal{V}_{S, i}^{2_{b} p} & =\xi_{p}^{1}(z) \gamma_{i}^{2_{b}}(z) S^{p}-\xi_{2_{b}}^{1}(z) \gamma_{i}^{p}(z) S^{2_{b}}=\gamma_{i}^{2_{b}}(z)\left(\xi_{p}^{1}(z) S^{p}-\xi_{2_{b}}^{1}(z) \nu_{p}(z) S^{2_{b}}\right) \\
& =\gamma_{i}^{2_{b}}(z) \mathcal{U}_{S}^{2_{b} p}
\end{aligned}
$$

The two $S^{1}$ terms, containing the nonholomorphic dependence of $\mathcal{V}_{S, i}^{2_{b} p}$ have cancelled out, leading to a holomorphic quantity which, moreover, is proportional to a $\mathcal{U}_{S}^{2_{b} p}$ form where $\mathcal{U}_{S}^{2_{b} p} \equiv \widetilde{\xi}_{p}^{2_{b}} S^{p}-\widetilde{\xi}^{p, 2_{b}} S^{2_{b}}$, cf. Eq. (B25), with the definitions $\widetilde{\xi}_{p}^{2} \equiv \xi_{p}^{1}(z)$ and $\widetilde{\xi}^{p, 2_{b}} \equiv \xi_{2_{b}}^{1}(z) \nu_{p}(z)$. The consistency of the latter definitions, see Eq. (B23), requiring the relation

$$
\frac{\widetilde{\xi}^{p, 2_{b}}}{\widetilde{\xi}_{p}^{2_{b}}}=\frac{\bar{W}_{M, p}\left(z^{\dagger}\right)}{\bar{W}_{M, 2_{b}}\left(z^{\dagger}\right)}
$$

follows immediately from (B41). This leads to the important conclusion that the function $\Xi_{i}$ in Eq. (B31) is in fact reduced to a function of only $\mathcal{U}_{S}$ 's with no dependence on $z^{\dagger}$ and where the $\gamma_{i}^{2 b}(z)$ factor in Eq. (B44) is absorbed in a redefinition of $\Xi_{i}$. Moreover, the $\mathcal{U}_{S}$ 's have to correspond at least to two disjoint sets of $S$-type fields since the relation (B44) is valid only for $p=2, \cdots, q_{0 b}-1$, while the initial $\mathcal{U}_{S}^{1 p}$ entries in Eq. (B31) correspond by construction to $p=q_{0 b}, \cdots, k_{1}$. One can easily check that the above properties established for $2_{b}$ are also valid for $2_{c}$ or for that matter any supplementary index $2_{d}$ of the same type. The general form should thus contain several disjoint $S$-field sets corresponding to disjoint sets of $\mathcal{U}_{S}$ entries.

Before summarizing the results we note that the linear contribution Eq. (B36) can now be recast in the form

$$
\sum_{p>1}^{k_{1}} \alpha_{p} \mathcal{V}_{S, j}^{2_{c} p}+\left(\frac{\partial \xi^{2_{c}}}{\partial z^{j *}}\right)^{-1} \frac{\partial}{\partial z^{j *}} \frac{f\left(z, z^{\dagger}\right)}{\bar{W}_{M, 1}\left(z^{\dagger}\right)} \mathcal{U}_{S}^{12_{c}}=\sum_{p \geq 1}^{k_{1}} W_{M-1, p}(z) S^{p}-\frac{f\left(z, z^{\dagger}\right)}{\bar{W}_{M, 1}(z \dagger)} S^{1}
$$

where we have used Eqs. (B39, B38, B16), the holomorphy of $\xi_{p}^{1}$ in $z$ and Eq. (B30). Note that the right-hand side of the above equation is independent of the $j$ index, in accordance with the requirement of Eq. (B36).

Putting all the pieces together we can now write down the general solutions for $W_{M}$ and $W_{M-1}$. This entails the choice of an arbitrary partition of the set of $k_{1} S$-type fields into $P$ disjoint subsets. We thus have

$$
k_{1}=\sum_{p \geq 1}^{P} n_{p}
$$

where $p=1, \ldots, P$ label the subsets and $n_{p}$ denotes the number of $S$-type fields forming the $p^{t h}$ subset. We also denote by $S^{p_{s}}$, with $s=1, \ldots, n_{p}$, the $S$-type fields of the $p^{t h}$ subset. With these notations the general solutions are summarized as follows:

$$
\begin{aligned}
W_{M}(z, S, \widetilde{\Phi}) & =W_{M, 0}(z)+\sum_{p \geq 1}^{P} W_{M, p}(z) \sum_{s \geq 1}^{n_{p}} \mu_{p_{s}}^{*} S^{p_{s}} \\
W_{M-1}(z, S, \widetilde{\Phi}) & =\sum_{q \geq 1}^{k_{1}} W_{M-1, q}(z) S^{q}+\Xi\left(\ldots, \mathcal{U}_{S}^{p p_{s}} \ldots ; \ldots, \widetilde{\Phi}^{a}, \ldots ; \ldots, z^{i}, \ldots\right),
\end{aligned}
$$

where $\Xi$ is an arbitrary function of all its entries,

$$
\mathcal{U}_{S}^{p p_{s}}=\xi_{p_{s}}(z) S^{p_{s}}-\xi^{p_{s}}(z) S^{p_{1}}, \text { with } p=1, \ldots, P, \text { and } s=1, \ldots, n_{p},
$$

and 
- $\xi_{p_{s}}(z)$ and $\xi^{p_{s}}(z)$ should satisfy:

$$
\mu_{p_{s}} \xi_{p_{s}}(z)=\mu_{p_{1}} \xi^{p_{s}}(z)
$$

normalized to $\xi_{p_{1}}(z)=\xi^{p_{1}}(z)$, (note the non-ambiguous simplified notation, $\xi_{p_{s}}^{p} \rightarrow \xi_{p_{s}}, \xi_{p}^{p_{s}} \rightarrow \xi^{p_{s}}$ ),

- $W_{M, 0}(z), W_{M, p}(z), W_{M-1, q}(z)$ and $\xi_{p_{s}}(z)\left(\right.$ or $\left.\xi^{p_{s}}(z)\right)$ are arbitrary holomophic functions of the $z^{i}$ fields,

- $\mu_{p_{s}}$ are arbitrary complex-valued constants,

- $P, n_{p}$, and $k_{1}$ are arbitrary positive integers satisfying Eq. (B47),

- $a=1, \ldots, k_{0}$ with $k_{0}$ an arbitrary positive integer, labels the $\widetilde{\Phi}$-type fields.

- $q=1, \ldots, k_{1}$ with $k_{1}$ an arbitrary positive integer, labels the $S$-type fields.

- $i=1, \ldots, \ell$ with $\ell$ an arbitrary positive integer, labels the $z$ fields.

We stress that while the $\mu_{p_{s}}$ 's enter Eq. (B51), it is their complex-conjugate that enter Eq. (B48). Note finally that the sum in Eq. (B49) is a shorthand notation for

$$
\sum_{q \geq 1}^{k_{1}} W_{M-1, q}(z) S^{q} \equiv \sum_{p \geq 1}^{P} \sum_{s \geq 1}^{n_{p}} W_{M-1, p_{s}}(z) S^{p_{s}} .
$$

We close this discussion by a few remarks - the result we arrived at implies that all the relevant $\xi$ 's are holomorphic in $z$. This is not to mean that it would have been equivalent to choosing from the start all the $\xi$ 's holomorphic in Eq. (B21). In the latter case all the $\mathcal{U}_{S}^{r, a}$ sets would be related to each other thanks to identities similar to Eq. (B20). The holomorphy structure in Eq. (B23) would then imply that the $\bar{W}_{M, a}\left(z^{\dagger}\right)$ are all expressed in terms of one single function of $z^{\dagger}$ up to constant factors. This is obviously less general than what we found through the above detailed analysis and corresponds simply to the special choice $P=1$ and $n_{1}=k_{1}$ in (B47), (B48), (B49), that is the trivial partition of the $S$-type fields set-the important point in our general result is that if some of the $\xi$ 's are not holomorphic then cancellations should take place separately in each entry of $\Xi$ in such a way that generates partitions of disjoint subsets of $S$-type fields - as stated after Eq. (B26) the proof leading to Eqs. (B48, B49) has been carried out in a simplified version of Eq. (B24) where just one set of $\mathcal{U}_{S}^{r p}$ entries was assumed for $\Omega$ with fixed $r=1$. It is legitimate to ask whether in the absence of this simplifying assumption one would get more general solutions. But we see no memory of this assumption in the final form of our solutions. In particular the $\Xi$ function contains all the $S$-type fields albeit in the form of partitions into disjoint subsets.

- a non SW solution: If we truncate the expansion in Eq. (2.15) at $M=1$, putting $W_{M}=0$ for all $M \geq 2$, then the above solutions give us the most general form of the superpotential,

$$
W(z, S, \widetilde{\Phi})=m_{p \ell} W_{1}(z, S, \widetilde{\Phi})+W_{0}(z, S, \widetilde{\Phi})
$$

where $W_{1}$ and $W_{0}$ are given respectively by Eq. (B48) and Eq. (B49) with $M=1$. Note that the SW solution Eq. (3.5) meets this one only in the case where the $S$-type fields sector is not present and $W_{2}(z):=0$, thus confirming that the SW form is not the most general.

When $M>1$ additional constraints must be studied originating from lower strictly positive $c$ powers of $m_{p \ell}$ in (B1) that we now consider.

- $\mathbf{c}=\mathbf{2 M}-\mathbf{2}$ : relevant when $M \geq 2$, the term with $c=2 M-2$ in the master equation (B1) gets contributions from the 0 -sum and the 2 -sum terms and leads to the constraint,

$$
\begin{gathered}
\left(\frac{\partial W_{M-2}}{\partial \Phi^{a}} \frac{\partial \bar{W}_{M}}{\partial \Phi^{a *}}+\text { h.c. }\right)+\frac{\partial W_{M-1}}{\partial \Phi^{a}} \frac{\partial \bar{W}_{M-1}}{\partial \Phi^{a *}}+\left(\mathcal{D}_{i} W_{M}\right)\left(\overline{\mathcal{D}}_{i} \bar{W}_{M}\right) \\
+\left(\Phi^{a} \frac{\partial W_{M}}{\partial \Phi^{a}} \bar{W}_{M}+\text { h.c. }\right)-3 W_{M} \bar{W}_{M} \sim_{\Phi} 0
\end{gathered}
$$


where we use from now on the shorthand notation,

$$
\begin{aligned}
& \mathcal{D}_{i} W \equiv \frac{\partial W}{\partial z^{i}}+z^{i *} W, \\
& \overline{\mathcal{D}}_{i} \bar{W} \equiv \frac{\partial \bar{W}}{\partial z^{i *}}+z^{i} \bar{W} .
\end{aligned}
$$

Recall that summation over repeated indices is understood. Applying $\frac{\partial}{\partial \widetilde{\Phi}^{b}} \frac{\partial}{\partial \widetilde{\Phi}^{b *}}$ to the above equation and using Eqs. (B48 B49) one obtains

$$
\left|\frac{\partial^{2} W_{M-1}}{\partial \Phi^{a} \partial \widetilde{\Phi}^{b}}\right|^{2}=\left|\frac{\partial^{2} \Xi}{\partial \Phi^{a} \partial \widetilde{\Phi}^{b}}\right|^{2}=0
$$

Since this constraint is a sum over positive definite terms, it is valid separately for fixed $a, b$. Applying it to the case $\Phi^{a} \equiv S^{p}$ implies,

$$
\frac{\partial^{2} \Xi}{\partial S^{p} \partial \widetilde{\Phi}^{b}}=0
$$

that is $\Xi$ should be a direct sum of exclusively $\widetilde{\Phi}$ and $S$ dependent functions

$$
\Xi=\widetilde{\Xi}\left(\ldots, \widetilde{\Phi}^{b}, \ldots ; z\right)+\Xi_{S}\left(\ldots, S^{p}, \ldots ; z\right) .
$$

Applying further Eq. (B57) in the case $\Phi^{a} \equiv \widetilde{\Phi^{a}}$ implies that $\widetilde{\Xi}$ should be linear in $\widetilde{\Phi}$ which we write as

$$
\widetilde{\Xi}=\widetilde{W}_{M-1, a}(z) \widetilde{\Phi}^{a}+\widetilde{W}_{M-1,0}(z) .
$$

Back to Eq. (B54) to which we apply now $\frac{\partial}{\partial S^{p}} \frac{\partial}{\partial S^{p *}}$, one finds

$$
\left|\frac{\partial^{2} W_{M-1}}{\partial \Phi^{a} \partial S^{p}}\right|^{2} \sim_{\Phi} 0
$$

Given Eqs. (B49, B59, B60), the above constraint is trivially satisfied in the case $\Phi^{a} \equiv \widetilde{\Phi}^{a}$, while the case $\Phi^{a} \equiv S^{q}$ leads to

$$
\left|\frac{\partial^{2} \Xi_{S}}{\partial S^{p} \partial S^{q}}\right|^{2} \sim_{\Phi} 0
$$

The function $\Xi_{S}$ is thus at most quadratic in the $S$ fields. This is sufficient but necessary as well as can be seen by applying again $\frac{\partial}{\partial S^{r}} \frac{\partial}{\partial S^{r *}}$ to the above equation to obtain

$$
\left|\frac{\partial^{3} \Xi_{S}}{\partial S^{p} \partial S^{q} \partial S^{r}}\right|^{2}=0
$$

Again, since this is a sum of positive definite terms then each term of the sum should be seperately zero and the above equation should hold also for any fixed set of $p, q, r$. Thus $\Xi_{S}$ should be necessarily of the form

$$
\Xi_{S}=\frac{1}{2} \sum_{p, q} W_{M-1, p q}(z) S^{p} S^{q}+\sum_{p} W_{M-1, p}^{(S)}(z) S^{p}+W_{M-1,0}(z)
$$

where the functions $W_{M-1, p}^{(S)}(z)$ and $W_{M-1, p q}(z)$, the latter defined to be symmetric in $p, q$, are arbitrary albeit related among each other in such a way that $\Xi_{S}$ is a function of the combinations $\mathcal{U}_{S}$ or $\mathcal{V}$ as dictated by Eq. (B49). Thus when $M \geq 2$ the function $W_{M-1}$ as given in Eq. (B49) is constrained further to a polynomial form,

$$
\begin{aligned}
W_{M-1}(z, S, \widetilde{\Phi})= & \frac{1}{2} \sum_{p, q} W_{M-1, p q}(z) S^{p} S^{q}+\sum_{p}\left(W_{M-1, p}(z)+W_{M-1, p}^{(S)}(z)\right) S^{p} \\
& +\widetilde{W}_{M-1, a}(z) \widetilde{\Phi}^{a}+W_{M-1,0}(z)
\end{aligned}
$$


where we kept distinct the two contributions to the linear part in $S^{p}$ since $W^{(S)}$ must have a specific form, while $W_{M-1,0}(z)+\widetilde{W}_{M-1,0}(z)$ is denoted by $W_{M-1,0}(z)$ without loss of generality. Although necessary, the above form is not yet sufficient to fulfill Eq. (B54). Injecting it back into Eq. (B54) and using Eq. (B48) one finds,

$$
\begin{gathered}
\sum_{p, q} A_{p q}\left(z, z^{\dagger}\right) S^{p} S^{q *}+\left[\sum_{p} B_{q}\left(z, z^{\dagger}\right) S^{q}+\bar{W}_{M, p}\left(z^{\dagger}\right) \frac{\partial}{\partial S^{p}} W_{M-2}\left(z, S^{r}, \widetilde{\Phi}^{a}\right)+\text { h.c. }\right] \\
\sim_{\Phi} 0
\end{gathered}
$$

with

$$
\begin{aligned}
& A_{p q}\left(z, z^{\dagger}\right)=\sum_{r} W_{M-1, r p} \bar{W}_{M-1, r q}+ \\
& \left(\frac{\partial W_{M, p}}{\partial z^{i}}+z^{i *} W_{M, p}\right)\left(\frac{\partial \bar{W}_{M, q}}{\partial z^{i *}}+z^{i} \bar{W}_{M, q}\right)-W_{M, p} \bar{W}_{M, q} \\
& B_{q}\left(z, z^{\dagger}\right)=\sum_{p} W_{M-1, p q}\left(\bar{W}_{M-1, p}+\bar{W}_{M-1, p}^{(S)}\right)+ \\
& \left(\frac{\partial W_{M, q}}{\partial z^{i}}+z^{i *} W_{M, q}\right)\left(\frac{\partial \bar{W}_{M, 0}}{\partial z^{i *}}+z^{i} \bar{W}_{M, 0}\right)-2 W_{M, q} \bar{W}_{M, 0}
\end{aligned}
$$

and we have absorbed the remaining $\widetilde{\Phi}$ - and $S$-independent contributions in the right-hand side of (B66). The latter equation is a partial differential equation in the still unknown function $W_{M-2}$ (and $\bar{W}_{M-2}$ ) with respect to the $S$ (and $S^{\dagger}$ ) fields. There is thus a priori no reason to zero the $B_{q}\left(z, z^{\dagger}\right)$ and $A_{p q}\left(z, z^{\dagger}\right)$ coefficients of the $S$ polynomial in order to match Eq. (B66). However since the term $\bar{W}_{M, p}\left(z^{\dagger}\right) \frac{\partial}{\partial S^{p}} W_{M-2}\left(z, S^{r}, \widetilde{\Phi}^{a}\right)$ and its hermitian conjugate present in Eq.(B66) are respectively holomorphic and anti-holomporphic in $S$, one has necessarily

$$
A_{l r}\left(z, z^{\dagger}\right)=0 \forall l, r
$$

as can be easily seen by acting with the operator $\frac{\partial}{\partial S^{l}} \frac{\partial}{\partial S^{r *}}$ on Eq.(B66). This equation reduces then to

$$
\sum_{p} B_{q}\left(z, z^{\dagger}\right) S^{q}+\bar{W}_{M, p}\left(z^{\dagger}\right) \frac{\partial}{\partial S^{p}} W_{M-2}\left(z, S^{r}, \widetilde{\Phi}^{a}\right)=g\left(z, z^{\dagger}\right) \sim_{\Phi} 0
$$

where $g$ denotes an arbitrary $\Phi$ independent function. ${ }^{11}$ This differential equation is similar to Eq. (B12) except that now we have a dependence on the variables $S^{r}$ in the nonhomogeneous part. Using well-known techniques in the theory of quasi-linear differential equations of first order we find the general solution ${ }^{12}$

$$
\begin{aligned}
W_{M-2}\left(z, S^{r}, \widetilde{\Phi}^{a}\right)= & \left(\frac{g\left(z, z^{\dagger}\right)}{\bar{W}_{M, 1}}-\sum_{q \geq 2} B_{q} S^{q}\right) S^{1}+\frac{1}{2}\left(\sum_{q \geq 2} \frac{\xi^{q}}{\xi^{1}} B_{q}-B_{1}\right)\left(S^{1}\right)^{2} \\
& +\Gamma\left(\ldots, \mathcal{U}_{S}^{1 q}, \ldots, \widetilde{\Phi}^{a} ; z\right) .
\end{aligned}
$$

11 That the h.c. in Eq. (B66) can be dropped in (B70) is intuitively clear, but can be seen by taking the derivative with respect to $S^{r}$ or with respect to $\widetilde{\Phi}^{a}$, showing respectively that the left-hand side of $(B 70)$ should be $\sim_{S} 0$ and $\sim_{\widetilde{\Phi}} 0$.

12 We refrain from detailing here the steps leading to Eq. (B71). Suffice it to recall that the general solution should be a function of an independent set of integrals of the characteristic ordinary differential equations system associated with Eq. (B70); for more details see e.g. $[52,53]$. The upshot is that the general solution to a multi-variable function $f\left(x_{1}, x_{2}, \ldots x_{n}\right)$ satisfying a differential equation of the form

$$
\sum_{i=1}^{n} a_{i} \frac{\partial f}{\partial x_{i}}=b\left(x_{1}, x_{2}, \ldots\right)
$$

where all the $a_{i}$ are $x$ independent and at least $a_{1}$ nonvanishing if $b$ is nonvanishing, is

$$
\begin{array}{r}
f\left(x_{1}, x_{2}, \ldots\right)=\frac{1}{a_{1}} \int_{x_{0}}^{x_{1}} d x b\left(x, \ldots, x_{i}-\frac{\xi^{i}}{\xi^{1}}\left(x_{1}-x\right), \ldots\right)+\mathcal{F}\left(\xi^{1} x_{2}-\xi^{2} x_{1}, \ldots,\right. \\
\left.\xi^{1} x_{i}-\xi^{i} x_{1}, \ldots, \xi^{1} x_{n}-\xi^{n} x_{1}\right)
\end{array}
$$

where $\xi^{1} a_{i}=\xi^{i} a_{1}, \mathcal{F}$ an arbitrary function of $n-1$ variables, and $x_{0}$ an arbitrary reference constant. 
Here $\mathcal{U}_{S}^{1 q}$ is as defined in Eq. (B25) and $\Gamma$ an arbitrary function. As in the case of Eq. (B14), we assumed when writing the above solution at least one nonzero $W_{M, p}$ to ensure that the set of fields of the $S$-type is not empty. However, as already mentioned after Eq. (B24), the case of no $S$-type fields is implicitly accounted for in the above equation by dropping out from it all the $S$ dependence. Note also that in the case of a single $S$-type field the summations in Eq. (B71) are trivially absent and $\Gamma$ becomes $S$-independent. Finally, one should in principle study further the constraint that the holomorphy of $W_{M-2}$ puts on Eq.(B71). However, it turns out that this is unnecessary to reach a definite conclusion, as the constraints originating from lower values of $c$ to be studied below are much more restrictive.

- $\mathbf{c}=\mathbf{2 M}-\mathbf{3}$ : relevant when $M \geq 2$, this term gets contributions from the 0-sum and the 2-sum terms. When $M \geq 3$ it leads to the constraint,

$$
\begin{gathered}
{\left[\frac{\partial W_{M}}{\partial \Phi^{a}} \frac{\partial \bar{W}_{M-3}}{\partial \Phi^{a *}}+\frac{\partial W_{M-2}}{\partial \Phi^{a}} \frac{\partial \bar{W}_{M-1}}{\partial \Phi^{a *}}+\left(\frac{\partial W_{M}}{\partial z^{i}}+z^{i *} W_{M}\right)\left(\frac{\partial \bar{W}_{M-1}}{\partial z^{i *}}+z^{i} \bar{W}_{M-1}\right)\right.} \\
\left.+\Phi^{a} \frac{\partial W_{M}}{\partial \Phi^{a}} \bar{W}_{M-1}+\Phi^{a} \frac{\partial W_{M-1}}{\partial \Phi^{a}} \bar{W}_{M}-3 W_{M} \bar{W}_{M-1}\right]+ \text { h.c. } \\
\sim_{\Phi} 0 .
\end{gathered}
$$

Specializing to $M=2$, the $W_{M-3}$ dependent term is absent by definition. Moreover, anticipating that $\frac{\partial W_{M}}{\partial \Phi^{a}}=0$ for $M \geq 3$, a result we will prove independently below (see Eq. (B88) in the $c=2 M-4$ case), then again the $W_{M-3}$ dependent term is absent in Eq. (B72). For the sake of conciseness we can thus safely replace in the subsequent discussion Eq. (B72) by

$$
\begin{gathered}
{\left[\frac{\partial W_{M-2}}{\partial \Phi^{a}} \frac{\partial \bar{W}_{M-1}}{\partial \Phi^{a *}}+\left(\frac{\partial W_{M}}{\partial z^{i}}+z^{i *} W_{M}\right)\left(\frac{\partial \bar{W}_{M-1}}{\partial z^{i *}}+z^{i} \bar{W}_{M-1}\right)\right.} \\
\left.+\Phi^{a} \frac{\partial W_{M}}{\partial \Phi^{a}} \bar{W}_{M-1}+\Phi^{a} \frac{\partial W_{M-1}}{\partial \Phi^{a}} \bar{W}_{M}-3 W_{M} \bar{W}_{M-1}\right]+ \text { h.c. } \\
\sim_{\Phi} 0,
\end{gathered}
$$

and use it for all $M \geq 2$. Note, though, that we kept concistently a $\frac{\partial W_{M}}{\partial \Phi^{a}}$ term (harmless when $M \geq 3$ ) since at this level it is not vanishing in the case $M=2$. Taking into account $W_{M}$ and $W_{M-1}$ given by Eqs. (B48), (B65), one rewrites (B73) as

$$
\begin{gathered}
{\left[\frac{\partial W_{M-2}}{\partial \Phi^{a}} \frac{\partial \bar{W}_{M-1}}{\partial \Phi^{a *}}+\frac{1}{2} K_{3, p q r} S^{p} S^{q} S^{r *}+K_{2, p r} S^{p} S^{r *}+\widetilde{K}_{2, b r} \widetilde{\Phi}^{b} S^{r *}\right.} \\
\left.+\frac{1}{2} K_{2, p q^{\prime}}^{\prime} S^{p} S^{q}+K_{1 r} S^{r *}+K_{1, p}^{\prime} S^{p}+\widetilde{K}_{1, b} \widetilde{\Phi}^{b}\right]+ \text { h.c. } \\
\sim_{\Phi} 0
\end{gathered}
$$

where the various $K$ factors are well-defined $S$-, $\widetilde{\Phi}$-independent functions of $z, z^{\dagger}$. Here we list only the ones relevant for the remainder of the discussion,

$$
\begin{aligned}
K_{3, p q r} & =\left(\mathcal{D}_{i} W_{M-1, p q}\right)\left(\overline{\mathcal{D}}_{i} \bar{W}_{M, r}\right) \\
\widetilde{K}_{2, b l} & =\left(\mathcal{D}_{i} \widetilde{W}_{M-1, b}\right)\left(\overline{\mathcal{D}}_{i} \bar{W}_{M, l}\right)-\widetilde{W}_{M-1, b} \bar{W}_{M, l} \\
\widetilde{K}_{1, b} & =\left(\mathcal{D}_{i} \widetilde{W}_{M-1, b}\right)\left(\overline{\mathcal{D}}_{i} \bar{W}_{M, 0}\right)-2 \widetilde{W}_{M-1, b} \bar{W}_{M, 0}
\end{aligned}
$$

Recall that the $a$ index in Eq. (B74) runs over all $S$ and $\widetilde{\Phi}$ fields. The holomorphy structure of this equation allows to write down through an appropriate choice of derivatives simple necessary constraints:

1) acting with the operator $\frac{\partial^{2}}{\partial S^{l *} \partial \widetilde{\Phi}^{b}}$ on Eq. (B74) with $b, l$ arbitrary fixed indices, one finds

$$
\bar{W}_{M-1, l p} \frac{\partial^{2} W_{M-2}}{\partial S^{p} \partial \widetilde{\Phi}^{b}}+\widetilde{K}_{2, b l}=0
$$


2) acting with the operator $\frac{\partial^{2}}{\partial S^{l} \partial S^{m} \partial S^{n *}}$ on Eq. (B74) with $l, m, n$ arbitrary fixed indices one finds

$$
\bar{W}_{M-1, n q} \frac{\partial^{3} W_{M-2}}{\partial S^{q} \partial S^{l} \partial S^{m}}+K_{3, n l m}=0 .
$$

Combined with Eqs. (B75, B76), these holomorphy constraints take the following form:

$$
\begin{gathered}
\bar{W}_{M-1, l p}\left(z^{\dagger}\right) \alpha_{p b}(z)=\left(\mathcal{D}_{i} \widetilde{W}_{M-1, b}(z)\right)\left(\overline{\mathcal{D}}_{i} \bar{W}_{M, l}\left(z^{\dagger}\right)\right)-\widetilde{W}_{M-1, b}(z) \bar{W}_{M, l}\left(z^{\dagger}\right), \\
\forall b, l,
\end{gathered}
$$

and

$$
\bar{W}_{M-1, r p}\left(z^{\dagger}\right) \alpha_{p q l}(z)=\left(\mathcal{D}_{i} W_{M-1, l q}(z)\right)\left(\overline{\mathcal{D}}_{i} \bar{W}_{M, r}\left(z^{\dagger}\right)\right), \quad \forall l, q, r .
$$

The last equation has exactly the form of Proposition A.4. As a result, the set of functions $W_{M-1, l q}(z)$ should all be vanishing if there exists at least one $r$ for which $W_{M, r}$ is nonzero. Injecting $W_{M-1, l q}(z)=0$ in (B67) leads through (B69) to

$$
\left(\mathcal{D}_{i} W_{M, p}(z)\right)\left(\overline{\mathcal{D}}_{i} \bar{W}_{M, q}\left(z^{\dagger}\right)\right)=W_{M, p}(z) \bar{W}_{M, q}\left(z^{\dagger}\right), \quad \forall p, q,
$$

which is of the form of Proposition A.1 with $\alpha=0, R=0$. However, in the special cases $p=q$ the above equation takes the form of Proposition A.1 with $P=Q \equiv W_{M, p}$. This implies through A.3 that all $W_{M, p}$ should be vanishing, in contradiction with our starting assumption that at least one of them is not. This shows that the only consistent result is

$$
\frac{\partial W_{M}}{\partial S^{p}}=W_{M, p}(z)=0, \forall p=1, \ldots, k_{1} .
$$

Recall that the proof of this result is strictly speaking valid here only for $M=2$.

$$
\text { It implies that there are no } S \text {-type fields when } M=2 \text {. }
$$

As we anticipated earlier, to prove its validity for $M>2$ one needs to consider the $c=2 M-4$ case to which we turn now.

- $\mathbf{c}=\mathbf{2 M}-\mathbf{4}$ : relevant when $M \geq 3$, this term gets contributions from the 0 -sum, 2-sum and 4-sum terms. When $M \geq 4$ it leads to the constraint,

$$
\begin{aligned}
& {\left[\frac{\partial W_{M}}{\partial \Phi^{a}} \frac{\partial \bar{W}_{M-4}}{\partial \Phi^{a *}}+\frac{\partial W_{M-1}}{\partial \Phi^{a}} \frac{\partial \bar{W}_{M-3}}{\partial \Phi^{a *}}+\left(\frac{\partial W_{M}}{\partial z^{i}}+z^{i *} W_{M}\right)\left(\frac{\partial \bar{W}_{M-2}}{\partial z^{i *}}+z^{i} \bar{W}_{M-2}\right)\right.} \\
& \left.+\Phi^{a} \frac{\partial W_{M}}{\partial \Phi^{a}} \bar{W}_{M-2}+\Phi^{a} \frac{\partial W_{M-1}}{\partial \Phi^{a}} \bar{W}_{M-1}+\Phi^{a} \frac{\partial W_{M-2}}{\partial \Phi^{a}} \bar{W}_{M}-3 W_{M} \bar{W}_{M-2}\right]+ \text { h.c. } \\
& +\left|\frac{\partial W_{M-2}}{\partial \Phi^{a}}\right|^{2}+\left|\frac{\partial W_{M-1}}{\partial z^{i}}+z^{i *} W_{M-1}\right|^{2}-3\left|W_{M-1}\right|^{2}+\left|W_{M}\right|^{2}\left|\Phi^{a}\right|^{2} \sim_{\Phi} 0
\end{aligned}
$$

and the special case $M=3$ is obtained by dropping in the above equation the contribution of $W_{M-4}$.

Acting with the operator $\frac{\partial}{\partial \widetilde{\Phi}^{b}} \frac{\partial}{\partial \widetilde{\Phi}^{b *}}$ where summation over the $b$ index is understood, on Eq. (B85) and using Eq. (B65) we obtain

$$
\left|\frac{\partial^{2} W_{M-2}}{\partial \Phi^{a} \partial \widetilde{\Phi}^{b}}\right|^{2}+\left|\frac{\partial \widetilde{W}_{M-1, b}}{\partial z^{i}}+z^{i *} \widetilde{W}_{M-1, b}\right|^{2}-2\left|\widetilde{W}_{M-1, b}\right|^{2}+\left|W_{M}\right|^{2}=0 .
$$

Applying to the above equation the operator $\frac{\partial}{\partial S^{q}} \frac{\partial}{\partial S^{q^{*}}}$ where summation over the $q$ index is understood, recalling that the $\widetilde{W}_{M-1, b}$ are $\widetilde{\Phi}$ and $S$ independent [cf. Eq. (B65) and using Eq. (B8) where by definition the $\Phi^{a}$ are only the $S$-type fields, or equivalently using Eq. (B48) for any chosen partition $P$, one finds a sum of squares satisfying

$$
\left|\frac{\partial^{3} W_{M-2}}{\partial S^{p} \partial \Phi^{a} \partial \widetilde{\Phi}^{b}}\right|^{2}+\left|W_{M, q}\right|^{2}=0
$$


that implies

$$
W_{M, q}=0, \forall q=1, \ldots, k_{1} .
$$

Here $W_{M, q}$ denotes generically all the $W_{M, p}(z) \mu_{p_{s}}$ factors appearing in Eq. (B48). The $S$-type set is thus empty when $M \geq 3$ and, given (B84), we conlude that:

$$
\text { there are no } S \text {-type fields when } M \geq 2 .
$$

The $W_{M-1}, W_{M-2}$ functions determined previously have thus no dependence on $S$-type fields. In particular the first sum on the left-hand side of Eq. (B87) vanishes trivially and there are no other constraints from this equation. Equations (B48, B65, B71) and (B74) simplify now to

$$
\begin{aligned}
W_{M}(z) & =W_{M, 0}(z) \\
W_{M-1}(z) & =\widetilde{W}_{M-1, a}(z) \widetilde{\Phi}^{a}+W_{M-1,0}(z), \\
W_{M-2}(z) & =\Gamma\left(\ldots, \widetilde{\Phi}^{a}, \ldots ; z\right)
\end{aligned}
$$

and

$$
\left[\frac{\partial W_{M-2}}{\partial \widetilde{\Phi}^{a}} \frac{\partial \bar{W}_{M-1}}{\partial \widetilde{\Phi}^{a *}}+\widetilde{K}_{1, a} \widetilde{\Phi}^{a}\right]+\text { h.c. } \sim_{\Phi} 0 .
$$

Operating $\frac{\partial}{\partial \widetilde{\Phi}^{b}}$ on this last equation yields,

$$
\left(\mathcal{D}_{i} \widetilde{W}_{M-1, b}\right)\left(\overline{\mathcal{D}}_{i} \bar{W}_{M, 0}\right)=2 \widetilde{W}_{M-1, b} \bar{W}_{M, 0}-\overline{\widetilde{W}}_{M-1, a} \frac{\partial^{2} \Gamma}{\partial \widetilde{\Phi}^{a} \partial \widetilde{\Phi}^{b}}
$$

where we have used (B91, B92) and (B77). Since $W_{M} \neq 0$ by assumption of the expansion (2.15), that is $\bar{W}_{M, 0} \neq 0$ cf. (B90), then Eq.(B94) which is of the form of Proposition A.4 implies

$$
\widetilde{W}_{M-1, b}=0, \forall b,
$$

meaning that for all $M \geq 2$ only $W_{M-2}$ can depend on $\widetilde{\Phi}$ fields. In the case $M=2$ there are no further constraints. However, when $M \geq 3$ the constraint (B86) becomes relevant and, combined with (B95), leads to a sum of squares implying $W_{M}=0$, a contradiction! Thus the expansion (2.15) should be truncated at most at $M=2$.

\section{Summary:}

- $M=1$ : the most general solution for the superpotential is

$$
W=m_{p \ell} W_{1}(z, S, \widetilde{\Phi})+W_{0}(z, S, \widetilde{\Phi}),
$$

where $W_{1}$ and $W_{0}$ are given by Eqs. (B48) and (B49) where all the involved functions are arbitrary,

- $M=2$ : the most general solution for the superpotential is $W=m_{p \ell}^{2} W_{2}(z)+m_{p \ell} W_{1}(z)+W_{0}(z, \widetilde{\Phi})$, where $W_{2}, W_{1}$ and $W_{0}$ are given by Eqs. (B90), (B91) with $\widetilde{W}_{M-1, a}=0$, and (B92), where all the involved functions are arbitrary,

- $M \geq 3$ : no solution.

This list exhausts all possibilities in the case of the minimal Kähler potential.

\section{General Kähler}

The situation when the Kähler potential is non-canonical is much more involved. In this appendix rather than studying completely the non-canonical case, we point out new difficulties which appear in this case and two peculiar solutions are be given. The resolution of the analogous of equation (3.3) for a general Kähler potential is much more difficult. Assuming that the Kähler potential and superpotential have the expansions given in Eqs. $(2.14,2.15)$, the actual computation of the $F$-term potential of Eq. (2.1) entails three main new difficulties in comparison with the canonical Kähler case: 
1. The inversion of the Kähler metric is performed perturbatively as an expansion in negative powers of $m_{p \ell}$; the actual form of the expansion depends on whether the observable sector enters explicitly $K_{n}$ or not.

2. Since $K^{i a^{*}}$ and $K^{a i^{*}}$ are in general different from zero, the coupling between the observable and the hidden sectors renders the constraints fulfilling the consistency requirement (2.12) much more involved.

3. As the Kähler potential is a polynomial of degree $N$ in the Planck mass, when $N>2$ Taylor expanding the exponential factor gives rise to new contributions.

Points 1 and 3 could lead to some conspiracy. Indeed, the expansion of the exponential factor leads to an unbounded from above series in $m_{p \ell}$ while the inverse of the Kähler metric leads to an unbounded from above series in $1 / m_{p \ell}$. This means that we could imagine some situations where these two contributions cancel each other. However, in general there is no complete cancellation between these two contributions and dangerous terms are generated. In our first analysis, in order to forbid dangerous terms, we consider the case where $N=2$. Three configurations must then be considered characterized by different explicit dependence of the functions $K_{n}, n=0,1,2$, on the observable sector as follows:

$$
\begin{aligned}
& K\left(Z, Z^{\dagger}\right)=m_{p \ell}^{2} K_{2}\left(z, z^{\dagger}, \Phi, \Phi^{\dagger}\right)+m_{p \ell} K_{1}\left(z, z^{\dagger}, \Phi, \Phi^{\dagger}\right)+K_{0}\left(z, z^{\dagger}, \Phi, \Phi^{\dagger}\right), \\
& K\left(Z, Z^{\dagger}\right)=m_{p \ell}^{2} K_{2}\left(z, z^{\dagger}\right)+m_{p \ell} K_{1}\left(z, z^{\dagger}, \Phi, \Phi^{\dagger}\right)+K_{0}\left(z, z^{\dagger}, \Phi, \Phi^{\dagger}\right), \\
& K\left(Z, Z^{\dagger}\right)=m_{p \ell}^{2} K_{2}\left(z, z^{\dagger}\right)+m_{p \ell} K_{1}\left(z, z^{\dagger}\right)+K_{0}\left(z, z^{\dagger}, \Phi, \Phi^{\dagger}\right) .
\end{aligned}
$$

Distinguishing these three cases is necessary in particular because they lead to different results for the perturbative inversion of the Kähler metric. Considering each case separately, with the superpotential given by (2.15), three master equations, analogous to the master equation in the flat case (B1), are obtained. In each situation new solutions are obtained.

Let us go into more details for the configuration given by (B96). In this case the inverse Kähler metric takes the form

$$
\begin{aligned}
K^{I J^{*}}= & \frac{1}{m_{p \ell}^{2}} K_{2}^{I J^{*}}-\frac{1}{m_{p \ell}^{3}} K_{2}^{I K^{*}} K_{1 K^{*} L} K_{2}^{L J^{*}} \\
& +\frac{1}{m_{p \ell}^{4}}\left(-K_{2}^{I K^{*}} K_{0 K^{*} L} K_{2}^{L J^{*}}+K_{2}^{I K^{*}} K_{1 K^{*} L} K_{2}^{L M^{*}} K_{1 M^{*} N} K_{2}^{N J^{*}}\right) \\
& +\mathcal{O}\left(1 / m_{p \ell}^{5}\right) \\
= & \frac{1}{m_{p \ell}^{2}} K_{2}^{I J^{*}}+\frac{1}{m_{p \ell}^{3}} \widetilde{K}_{-3}^{I J^{*}}+\frac{1}{m_{p \ell}^{4}} \widetilde{K}_{-4}^{I J^{*}}+\mathcal{O}\left(1 / m_{p \ell}^{5}\right)
\end{aligned}
$$

where

$$
K_{2}^{I J^{*}}=\left(\frac{\partial^{2} K_{2}}{\partial Z^{I} \partial Z^{J^{*}}}\right)^{-1}
$$

Particularizing to the case where $M=2$, the $F$-term contribution to the scalar potential takes the form

$$
\begin{aligned}
& V_{F}=e^{K_{2}+\frac{1}{m_{p \ell}} K_{1}+\frac{1}{m_{p \ell}^{2}} K_{0}} \times \\
& \left(m_{p \ell}^{2}\left[\mathcal{D}_{I} W_{2} K_{2}^{I J^{*}} \mathcal{D}_{J *} \bar{W}_{2}-3\left|W_{2}\right|^{2}\right]+m_{p \ell}\left[\mathcal{D}_{I} W_{2} \widetilde{K}_{-3}^{I J^{*}} \mathcal{D}_{J *} \bar{W}_{2}\right.\right. \\
& \left.+\left(\mathcal{D}_{I} W_{2} K_{2}^{I J^{*}}\left(\mathcal{D}_{J *} \bar{W}_{1}+\bar{W}_{2} \frac{\partial K_{1}}{\partial Z^{J^{*}}}\right)+\text { h.c. }\right)-3\left(W_{2} \bar{W}_{1}+W_{1} \bar{W}_{2}\right)\right] \\
& +\mathcal{D}_{I} W_{2} \widetilde{K}_{-4}^{I J^{*}} \mathcal{D}_{J *} \bar{W}_{2}+\left(\mathcal{D}_{I} W_{2} \widetilde{K}_{-3}^{I J^{*}}\left(\mathcal{D}_{J^{*}} \bar{W}_{1}+\bar{W}_{2} \frac{\partial K_{1}}{\partial Z^{J^{*}}}\right)+\text { h.c. }\right) \\
& +\left(\mathcal{D}_{I} W_{1}+W_{2} \frac{\partial K_{1}}{\partial Z^{I}}\right) K_{2}^{I J^{*}}\left(\mathcal{D}_{J^{*}} \bar{W}_{1}+\bar{W}_{2} \frac{\partial K_{1}}{\partial Z^{J^{*}}}\right) \\
& \left.-3\left(W_{2} \bar{W}_{0}+W_{1} \bar{W}_{1}+W_{0} \bar{W}_{2}\right)+\mathcal{O}\left(\frac{1}{m_{p \ell}}\right)\right)
\end{aligned}
$$

where we have denoted

$$
\begin{aligned}
& \mathcal{D}_{I} W_{2}=\frac{\partial W_{2}}{\partial Z^{I}}+W_{2} \frac{\partial K_{2}}{\partial Z^{I}}, \\
& \mathcal{D}_{I} W_{1}=\frac{\partial W_{1}}{\partial Z^{I}}+W_{1} \frac{\partial K_{2}}{\partial Z^{I}}
\end{aligned}
$$


As in the flat case we must now impose that the terms in $m_{p \ell}^{2}$ and $m_{p \ell}$ do not depend on the observable sector. Rather than solving these complicated equations we now exhibit two specific solutions.

If we assume that

$$
\begin{aligned}
& K_{1}=0 \\
& W_{1}=0
\end{aligned}
$$

then $\widetilde{K}_{-3}=0$ and the term proportional to $m_{p \ell}$ drops out from $V_{F}$, leaving us with the dangerous term proportional to $m_{p \ell}^{2}$. To meet the requirement $(2.12)$, it is then sufficient to ask that $W_{2}$ and $K_{2}$ satisfy the condition

$$
\mathcal{D}_{I} W_{2} K_{2}^{I J^{*}} \mathcal{D}_{J *} \bar{W}_{2}-3\left|W_{2}\right|^{2} \sim_{\Phi} 0
$$

leaving the functions $K_{0}$ and $W_{0}$ totally free. the potential then reduces to

$$
\begin{array}{r}
V=e^{K_{2}+\frac{1}{m_{p \ell}^{2}} K_{0}}\left(m_{p \ell}^{2} f\left(z, z^{\dagger}\right)+\mathcal{D}_{I} W_{2} \widetilde{K}_{-4}^{I J^{*}} \mathcal{D}_{J} \bar{W}_{2}-3\left(W_{2} \bar{W}_{0}+W_{0} \bar{W}_{2}\right)\right. \\
\left.+\mathcal{O}\left(\frac{1}{m_{p \ell}}\right)\right)
\end{array}
$$

where $f$ denotes an arbitrary function of the hidden sector fields only. Having no dangerous terms this solution is an acceptable one, not found in [17]. Equation (B104) can be in principle solved as a partial differential equation for $W_{2}$ along the same lines as the analysis done in B 1. Note in particular that if we choose $f\left(z, z^{\dagger}\right) \equiv 0$ then Eq. (B104) reduces to a no-scale-like condition [26-29] involving the leading terms of the Kähler potential and superpotential.

Another special solution can be found as follows: suppose that

$$
W_{2}=0
$$

and let the functions $K_{0}, K_{1}, K_{2}$ and $W_{1}, W_{0}$ be totally free. Then the potential simplifies significantly to,

$$
V=e^{K_{2}+\frac{1}{m_{p \ell}} K_{1}+\frac{1}{m_{p \ell}^{2}} K_{0}}\left(\mathcal{D}_{I} W_{1} K_{2}^{I J^{*}} \mathcal{D}_{J *} \bar{W}_{1}-3\left|W_{1}\right|^{2}+\mathcal{O}\left(\frac{1}{m_{p \ell}}\right)\right)
$$

and no constraint are needed in order to obtain an acceptable solution. This second solution was not found in [17] either. If in addition we impose a no-scale-like condition [26-29],

$$
\mathcal{D}_{I} W_{1} K_{2}^{I J^{*}} \mathcal{D}_{J} \bar{W}_{1}-3\left|W_{1}\right|^{2}=0
$$

then the potential is naturally at the order $1 / m_{p \ell}$. Thus when Supergravity is broken the cosmological constant has a $1 / m_{p \ell}$ suppression.

The two new solutions above were obtained by simply putting to zero all dangerous terms. Proceeding as in the canonical case by solving the tower of differential equations, we anticipate the occurrence of other new solutions. A detailed study goes beyond the scope of this paper.

[1] ATLAS Collaboration, "Observation of a new particle in the search for the Standard Model Higgs boson with the ATLAS detector at the LHC," Phys. Lett. B716 (2012) 1-29, arXiv:1207.7214 [hep-ex] .

[2] CMS Collaboration, "Observation of a new boson at a mass of $125 \mathrm{GeV}$ with the CMS experiment at the LHC," Phys. Lett. B 716 (2012) 30-61, arXiv:1207.7235 [hep-ex].

[3] F. Englert and R. Brout, "Broken Symmetry and the Mass of Gauge Vector Mesons," Phys. Rev. Lett. 13 (1964) 321.

[4] P. W. Higgs, "Broken Symmetries and the Masses of Gauge Bosons," Phys. Rev. Lett. 13 (1964) 508.

[5] P. W. Higgs, "Broken Symmetries, Massless Particles and Gauge Fields," Phys. Lett. 12 (1964) 132.

[6] G. S. Guralnik, C. R. Hagen, and T. B. W. Kibble, "Global Conservation Laws and Mass-less Particles," Phys. Rev. Lett. 13 (1964) 585.

[7] A. H. Chamseddine, R. L. Arnowitt, and P. Nath Phys. Rev. Lett. 49 (1982) 970.

[8] R. Barbieri, S. Ferrara, and C. A. Savoy, "Gauge models with spontaneously broken local supersymmetry," Phys. Lett. B119 (1982) 343.

[9] L. E. Ibañez, "Locally supersymmetric $S U(5)$ grand unification," Phys. Lett. B118 (1982) 73.

[10] N. Ohta, "Grand Unified Theories Based on Local Supersymmetry," Prog. Theor. Phys. 70 (1983) 542. 
[11] J. R. Ellis, D. V. Nanopoulos, and K. Tamvakis, "Grand unification in simple supergravity," Phys. Lett. B121 (1983) 123.

[12] L. Alvarez-Gaumé, J. Polchinski, and M. B. Wise, "Minimal low-energy supergravity," Nucl. Phys. B221 (1983) 495.

[13] J. Polonyi, "Generalization of the Massive Scalar Multiplet Coupling to the Supergravity,". Hungary Central Inst Res KFKI-77-93 (unpublished).

[14] E. Cremmer, P. Fayet, and L. Girardello, "Gravity Induced Supersymmetry Breaking and Low-Energy Mass Spectrum," Phys. Lett. B122 (1983) 41.

[15] H. P. Nilles, M. Srednicki, and D. Wyler, "Weak Interaction Breakdown Induced by Supergravity," Phys. Lett. B120 (1983) 346.

[16] H. P. Nilles, "Supergravity Generates Hierarchies," Nucl. Phys. B217 (1983) 366.

[17] S. K. Soni and H. A. Weldon Phys.Lett. B126 (1983) 215.

[18] G. Giudice and A. Masiero Phys.Lett. B206 (1988) 480-484.

[19] H. P. Nilles, "Supersymmetry, Supergravity and Particle Physics," Phys. Rept. 110 (1984) 1-162.

[20] L. J. Hall, J. D. Lykken, and S. Weinberg, "Supergravity as the Messenger of Supersymmetry Breaking," Phys. Rev. D27 (1983) 2359-2378.

[21] A. Brignole, L. E. Ibanez, and C. Munoz, "Soft supersymmetry breaking terms from supergravity and superstring models," Adv. Ser. Direct. High Energy Phys. 21 (2010) 244-268.

[22] D. J. H. Chung, L. L. Everett, G. L. Kane, S. F. King, J. D. Lykken, and L.-T. Wang, "The Soft supersymmetry breaking Lagrangian: Theory and applications," Phys. Rept. 407 (2005) 1-203, arXiv:hep-ph/0312378 [hep-ph].

[23] T. Banks, D. B. Kaplan, and A. E. Nelson, "Cosmological implications of dynamical supersymmetry breaking," Phys. Rev. D49 (1994) 779-787, arXiv:hep-ph/9308292 [hep-ph].

[24] J. Wess and J. Bagger, Supersymmetry and Supergravity. Princeton University Press, revised ed., 3, 1992.

[25] R. Knoops, Phenomenological aspects of supergravity theories in de Sitter vacua. PhD thesis, KU Leuven, Dept. Phys. Astron., 2016. arXiv:1608.08841 [hep-ph].

[26] E. Cremmer, S. Ferrara, C. Kounnas, and D. V. Nanopoulos Phys.Lett. B133 (1983) 61.

[27] J. R. Ellis, C. Kounnas, and D. V. Nanopoulos Nucl.Phys. B247 (1984) 373-395.

[28] J. R. Ellis, A. Lahanas, D. V. Nanopoulos, and K. Tamvakis, "No-scale supersymmetric standard model," Phys.Lett. B134 (1984) 429.

[29] A. Lahanas and D. V. Nanopoulos Phys.Rept. 145 (1987) 1.

[30] P. Fayet, "Supergauge Invariant Extension of the Higgs Mechanism and a Model for the electron and Its Neutrino," Nucl. Phys. B90 (1975) 104-124.

[31] P. Fayet, "Spontaneously Broken Supersymmetric Theories of Weak, Electromagnetic and Strong Interactions," Phys. Lett. B69 (1977) 489.

[32] U. Ellwanger, C. Hugonie, and A. M. Teixeira, "The Next-to-Minimal Supersymmetric Standard Model," Phys. Rept. 496 (2010) 1-77, arXiv:0910.1785 [hep-ph].

[33] P. Fayet, "Massive gluinos," Phys. Lett. B78 (1978) 417.

[34] S. Dimopoulos and S. Raby, "Supercolor," Nucl. Phys. B192 (1981) 353.

[35] M. Dine, W. Fischler, and M. Srednicki, "Supersymmetric technicolor," Nucl. Phys. B189 (1981) 575.

[36] J. P. Derendinger and C. A. Savoy, "Gaugino masses and a new mechanism for proton decay in supersymmetric theories," Phys. Lett. B118 (1982) 347.

[37] M. Dine and W. Fischler, "A phenomenological model of particle physics based on supersymmetry," Phys. Lett. B110 (1982) 227.

[38] C. R. Nappi and B. A. Ovrut, "Supersymmetric extension of the $S U(3) \times S U(2) \times U(1)$ model," Phys. Lett. B113 (1982) 175.

[39] L. Alvarez-Gaumé, M. Claudson, and M. B. Wise, "Low-energy supersymmetry," Nucl. Phys. B207 (1982) 96.

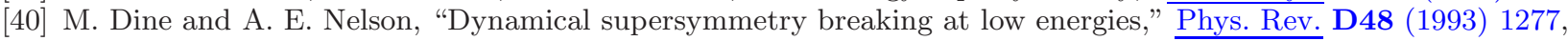
arXiv:hep-ph/9303230.

[41] M. Dine, A. E. Nelson, and Y. Shirman, "Low energy dynamical supersymmetry breaking simplified," Phys. Rev. D51 (1995) 1362, arXiv:hep-ph/9408384.

[42] M. Dine, A. E. Nelson, Y. Nir, and Y. Shirman, "New tools for low energy dynamical supersymmetry breaking," Phys. Rev. D53 (1996) 2658, arXiv:hep-ph/9507378.

[43] G. F. Giudice and R. Rattazzi, "Theories with gauge-mediated supersymmetry breaking," Phys. Rept. 322 (1999) 419, arXiv:hep-ph/9801271.

[44] N. Arkani-Hamed and N. Weiner, "LHC Signals for a SuperUnified Theory of Dark Matter," JHEP 12 (2008) 104, arXiv:0810.0714 [hep-ph].

[45] L. Girardello and M. T. Grisaru, "Soft Breaking of Supersymmetry," Nucl. Phys. B194 (1982) 65.

[46] S. J. Gates, M. T. Grisaru, M. Rocek, and W. Siegel, Superspace Or One Thousand and One Lessons in Supersymmetry, vol. 58. Front. Phys., 1983.

[47] M. T. Grisaru, W. Siegel, and M. Rocek, "Improved Methods for Supergraphs," Nucl. Phys. B159 (1979) 429.

[48] L. J. Hall, K. Jedamzik, J. March-Russell, and S. M. West, "Freeze-In Production of FIMP Dark Matter," JHEP 03 (2010) 080, arXiv:0911.1120 [hep-ph].

[49] G. Jungman, M. Kamionkowski, and K. Griest, "Supersymmetric dark matter," Phys. Rept. 267 (1996) 195-373, arXiv:hep-ph/9506380 [hep-ph].

[50] M. B. Einhorn and D. R. T. Jones, "Inflation with Non-minimal Gravitational Couplings in Supergravity," JHEP 03 
(2010) 026, arXiv:0912.2718 [hep-ph].

[51] S. Ferrara, R. Kallosh, A. Linde, A. Marrani, and A. Van Proeyen, "Jordan Frame Supergravity and Inflation in NMSSM," Phys. Rev. D82 (2010) 045003, arXiv:1004.0712 [hep-th].

[52] R. Courant and D. Hilbert, Methods of Mathematical Physics, Vol. 2. Wiley-VCH, volume 2 ed., $1,1989$.

[53] M. Decuyper and J. Kuntzmann, Modèles mathématiques de la physique: maîtrise d'électronique, d'électrotechnique et d'automatique, C1-ondes et matière. Dunod université. Dunod, 1972. https://books.google.fr/books?id=V5miOgAACAAJ.

[54] G. Moultaka, M. Rausch de Traubenberg, and D. Tant, "Low Energy Supergravity Revisited (I)," arXiv:1611.10327v1 [hep-th]. 